NBER WORKING PAPER SERIES

\title{
NO-ARBITRAGE SEMI-MARTINGALE RESTRICTIONS FOR CONTINUOUS-TIME VOLATILITY MODELS SUBJECT TO LEVERAGE EFFECTS, JUMPS AND I.I.D. NOISE: THEORY AND TESTABLE DISTRIBUTIONAL IMPLICATIONS
}

\author{
Torben G. Andersen \\ Tim Bollerslev \\ Dobrislav Dobrev
}

Working Paper 12963

http://www.nber.org/papers/w12963

\author{
NATIONAL BUREAU OF ECONOMIC RESEARCH \\ 1050 Massachusetts Avenue \\ Cambridge, MA 02138 \\ March 2007
}

This research was supported by a grant from the National Science Foundation to the NBER for Andersen and Bollerslev. We would like to thank two anonymous referees, Christian Bontemps, and Nour Meddahi for many helpful suggestions, which greatly improved the papers. We also thank participants at the International Finance Conference at the University of Copenhagen, September 2005, and the Time Series Conference at the University of Montreal, Canada, December 2005, as well as seminar participants at University of Maryland, Robert H. Smith School, University of Wisconsin, Madison; and the University of Chicago. The views expressed herein are those of the author(s) and do not necessarily reflect the views of the National Bureau of Economic Research.

(C) 2007 by Torben G. Andersen, Tim Bollerslev, and Dobrislav Dobrev. All rights reserved. Short sections of text, not to exceed two paragraphs, may be quoted without explicit permission provided that full credit, including $\odot$ notice, is given to the source. 
No-Arbitrage Semi-Martingale Restrictions for Continuous-Time Volatility Models Subject to Leverage Effects, Jumps and i.i.d. Noise: Theory and Testable Distributional Implications Torben G. Andersen, Tim Bollerslev, and Dobrislav Dobrev

NBER Working Paper No. 12963

March 2007

JEL No. C15,C22,C52,C80,G10

\begin{abstract}
$\underline{\text { ABSTRACT }}$
We develop a sequential procedure to test the adequacy of jump-diffusion models for return distributions. We rely on intraday data and nonparametric volatility measures, along with a new jump detection technique and appropriate conditional moment tests, for assessing the import of jumps and leverage effects. A novel robust-to-jumps approach is utilized to alleviate microstructure frictions for realized volatility estimation. Size and power of the procedure are explored through Monte Carlo methods. Our empirical findings support the jump-diffusive representation for S\&P500 futures returns but reveal it is critical to account for leverage effects and jumps to maintain the underlying semi-martingale assumption.

Torben G. Andersen

Kellogg School of Management

Northwestern University

2001 Sheridan Road

Evanston, IL 60208

and NBER and CREATES

t-andersen@kellogg.northwestern.edu

Tim Bollerslev

Department of Economics

Duke University

Box 90097

Durham, NC 27708-0097

and NBER and CREATES

boller@econ.duke.edu

Dobrislav Dobrev

Dept. of Finance, Kellogg School of Management Northwestern University 2001 Sheridan Road, Evanston, IL 60208

d-dobrev@kellogg.northwestern.edu
\end{abstract}




\section{Introduction}

Financial market volatility research has thrived over the last decades. New insights have been generated about as fast as data accessibility and computational power allowed the focus to shift from quarterly and monthly frequencies to daily data series. It is therefore striking that the recent widespread availability of a decades worth of high-frequency intraday data for a broad cross-section of actively traded financial assets has not fundamentally altered the landscape. $\mathrm{ARCH}$ and related stochastic volatility models implemented at the daily level remain the workhorses for practical volatility modeling. This reflects the limited progress achieved in using intraday data directly for volatility modeling and forecasting over the longer daily and monthly horizons of primary interest in applications. Of course, a variety of market microstructure and announcement studies exploit high-frequency data to great effect, but these dense data sets have still not been fully harnessed in regards to their implications concerning lower frequency return dynamics. Meanwhile, some promising alternatives relying on summary statistics extracted from intraday data have been entertained, for example models using daily ranges, e.g., Garman and Klass (1980), Parkinson (1980), Gallant, Hsu and Tauchen (1999) and Alizadeh, Brandt and Diebold (2002) and - very recently - the so-called realized volatility or variation measures, e.g., Andersen and Bollerslev (1998), Andersen, Bollerslev, Diebold and Labys (henceforth ABDL) (2001, 2003), Barndorff-Nielsen and Shephard (henceforth BN-S) (2002a,b), and Meddahi (2002). Substantial advances have been obtained through such approaches, and the construction of improved daily volatility measures from ultra-high frequency data is currently an active research area, see, e.g., Hansen and Lunde (2006) for an overview.

This article sheds further light on the characteristics of high-frequency asset return processes and their implications for daily return distributions. Our contribution is best appreciated in the context of the widely documented finding that the conditional distribution of the daily return innovation in standard volatility models invariably is heavy tailed and possesses extreme outliers. It is well known that ARCH models provide consistent volatility filters - as the underlying data is sampled at ever finer frequencies for extracting the conditional variance process from return series driven by a pure diffusion; see, e.g., Nelson $(1990,1992)$. Within this setting, the returns are locally conditionally Gaussian, so one may intuitively reason that the daily returns, appropriately standardized by the (realized) volatility over the course of the trading day, should be Gaussian as well. Furthermore, empirically, it has been found that this standardization produces normalized returns that indeed appear approximately Gaussian, although formal tests still typically reject normality fairly convincingly; see ABDL (2000, 2001), and Andersen, Bollerslev Diebold and Ebens (2001). This points to the potential usefulness of the above result, while also indicating that there may be features in the actual data which invalidate the underlying intuition.

One such critical feature is the presence of an asymmetric relation between the high-frequency return and volatility innovations, as implied by the so-called leverage or volatility feedback effects; see, 
e.g., the recent discussions in Gençay and Selçuk (2004) and Bollerslev, Litvinova and Tauchen (2005). If such an asymmetric relation is at work, the result should fail in theory, even if the underlying process is a continuous semi-martingale. However, through the "time-change theorem for continuous local martingales" we may restore the Gaussianity of appropriately standardized returns by sampling prices in "event" or "financial" time, as given by equal sized increments to the volatility process rather than in calendar time, i.e., equidistant time intervals. This result is valid for all (no-arbitrage) diffusive semimartingale representations of the price process and, importantly, does not rely on any data beyond the prices themselves. This observation has also been used by Peters and de Vilder (henceforth PV) (2004) and has a remarkable precedent in largely unnoticed work by Zhou (1998) who deals, heuristically, with both i.i.d. noise and leverage. The basic notion is also invoked in Ané and Geman (2000). ${ }^{1}$

The above scenarios largely exhaust the relevant possibilities when the underlying asset return process evolves as a continuous semi-martingale. Meanwhile, there is an increasing body of empirical work which concludes that continuous-time models must incorporate jumps or dis-continuities in order to provide a satisfactory characterization of the daily return process. Although the jump-diffusion setting is fully compatible with the no-arbitrage framework of financial asset pricing theory, as detailed in e.g., Back (1991), the presence of jumps take us outside the domain of the statistical framework and the timechange theorem discussed above. However, recent advances in the realized volatility literature include nonparametric data-driven procedures explicitly designed to identify jumps from underlying highfrequency return series; see BN-S (2004, 2005), Andersen, Bollerslev and Diebold (henceforth ABD) (2005), and Huang and Tauchen (2005). This suggests that we, following a preliminary jump detection and extraction step, may be able to apply the above reasoning and directly explore if the appropriately normalized trading day returns, adjusted for jumps, are Gaussian. However, constructing a jump-adjusted intraday return series requires knowledge not only of whether a jump occurred on a given trading day, but also about the number of jumps and the exact intervals during which they manifest themselves. In order to accomplish this task, we develop a novel jump detection procedure which we - surprisingly find to perform better than the regular BN-S inspired technique for a number of realistic scenarios.

In combination, our approach constitutes a novel sequential procedure for exploring whether a jump-diffusion offers a reasonable characterization of the return generating process. It also raises the

1 Ané and Geman ultimately rely on the number of transactions to characterize the event time evolution. In our model-free context, it is particularly important to avoid such auxiliary variables, as the imputed normality then invariably depends on additional model assumptions, see also the Gillemot, Farmer and Lillo (2005) discussion and critique of the empirical findings in Ané and Geman (2000). 
question of how the standardized returns in event time behave if the underlying price path exhibit jumps. Furthermore, do we have the power to detect jumps through existing identification techniques?

More generally, information regarding the strength of jump intensities and sizes, the significance and magnitude of leverage effects, along with direct estimates of the time series of diffusive volatility, are of immediate import for an array of important issues, including the causes behind extreme return realizations, the general risk-return tradeoff and the associated pricing of financial assets, the portfolio allocation problem, the construction of improved risk management techniques, and derivatives pricing. The possibility to gain insights into these issues through direct analysis of intraday return series under minimal auxiliary assumptions is intriguing. We address these issues through an empirical illustration based on a 2-minute intraday S\&P 500 futures return series covering a relatively long sample period from 1988 to 2004, and through an extensive simulation study. Finally, we also explore the impact of market microstructure noise and the intraday volatility pattern on our findings. For the former, we introduce a simple robust-to-jumps technique which is necessary for meaningful analysis of i.i.d. noise in our setting.

The paper progresses as follows. Section 2 provides additional motivation and formally outlines the framework. Section 3 explores the finite sample behavior of our new sequential test procedure for assessing the adequacy of the standard jump diffusive framework. We explore the properties of tests for empirically calibrated models under a variety of scenarios, including pure diffusions and jump-diffusions with and without leverage and microstructure noise. Section 4 presents our empirical analysis for the S\&P500 futures returns. We find strong evidence for the presence of both jumps and leverage effects. Moreover, we cannot to reject the hypothesis that the jump-adjusted and appropriately standardized event time returns is i.i.d. Gaussian. As such, our findings are consistent with asset prices being generated by an arbitrage-free jump-diffusive process. We also conclude that a pure diffusive representation, as in PV (2004), is unlikely to provide an adequate characterization of the price process. Section 5 concludes.

\section{Theoretical Background}

We let the logarithmic asset price process evolve according to a generic jump-diffusion. Even if the underlying prices cannot be observed at every instant, the recorded quote and transaction prices may be seen as, possibly noisy, observations from this continuously evolving process. Moreover, the sudden release of news or arrival of orders will often induce a distinct shift, or jump, in the asset price. The jump-diffusive setting is also theoretically appealing as it implies, under standard regularity, that the price process constitutes a special semi-martingale, thus ruling out arbitrage, see, e.g., Back (1991). Furthermore, it enables us, in principle, to derive the distribution of discretely observed returns at any 
frequency through aggregation, or integration, of the increments to the underlying process. Finally, it is a flexible setting with the potential to accommodate all major features of daily financial return series.

Our foremost interest is in gaining insight into the descriptive validity of the semi-martingale representation for asset prices as embodied within the general jump-diffusion setting. The main limitation is that we exclude Lévy jump processes with infinite jump intensity, see, e.g., Carr, Geman, Madan and Yor (2002), as we only allow for "rare" jumps occurring at a finite rate. Hence, key issues are to what extent the jump-diffusion representation is consistent with empirical data and what specific features of the specification are necessary in order to adequately describe the observed return processes.

\subsection{Quadratic Variation, Realized Volatility, and Trading Day Return Distributions}

We focus on the univariate case. Let $p(t)$ denote the time $t$ logarithmic asset price. The generic jumpdiffusion process may then be expressed in stochastic differential equation (sde) form,

$$
d p(t)=\mu(t) d t+\sigma(t) d W(t)+\kappa(t) d q(t), \quad 0 \leq t \leq T,
$$

where $\mu(t)$ is a locally bounded variation process, the volatility process $\sigma(t)$ is strictly positive and càglàd, ${ }^{2} W(t)$ is a Wiener process, $d q(t)$ is a counting process with $d q(t)=1$ if there is a jump at time $\mathrm{t}$ and $d q(t)=0$ otherwise with (possibly time-varying) jump intensity $\lambda(t)$, and $\kappa(t)$ refers to the corresponding jump size. The quadratic variation of the cumulative return process, $r(t) \equiv p(t)-p(0)$, is given by

$$
[r, r]_{t}=\int_{0}^{t} \sigma^{2}(s) d s+\sum_{0<s \leq t} \kappa^{2}(s)
$$

Let the discretely sampled $\Delta$-period returns be denoted by, $r_{t, \Delta} \equiv p(t)-p(t-\Delta)$. The daily realized volatility is then defined by the summation of the corresponding $1 / \Delta$ high-frequency intraday squared returns,

$$
R V_{t+1}(\Delta) \equiv \sum_{j=1}^{1 / \Delta} r_{t+j \cdot \Delta, \Delta}^{2},
$$

where, without loss of generality, $1 / \Delta$ denotes an integer. As emphasized by Andersen and Bollerslev (1998), ABDL (2001, 2003), BN-S (2002a,b) and Comte and Renault (1998), among others, by the theory of quadratic variation this realized volatility converges uniformly in probability, under weak

\footnotetext{
2 This assumption allows for discrete jumps in the volatility process. Recent work on Lévy-driven stochastic volatility models include BN-S (2001), Carr, Geman, Madan and Yor (2003), and Todorov and Tauchen (2005). 
regularity, to the corresponding increment to the quadratic variation process, i.e. for $\Delta \rightarrow 0$,

$$
R V_{t+1}(\Delta) \rightarrow[r, r]_{t+1}-[r, r]_{t}=\int_{t}^{t+1} \sigma^{2}(s) d s+\sum_{t<s \leq t+1} \kappa^{2}(s)
$$

In the absence of jumps, the realized volatility is thus consistent for the so-called integrated variance and, importantly, if jumps are present the realized volatility is consistent for the sum of the integrated variance and the cumulative sum of squared jumps. Hence, the realized volatility approximates (for $\Delta>0$ ) the total (ex-post) return variability, whether the source is the diffusive or the jump component of the return process. Note also that the drift (mean) process has no effect on the limiting result in (4). Throughout, we will be operating with sufficiently high-frequency return series and short return horizons such that the mean process can be safely ignored. Thus, for simplicity, we set $\mu(t)=0$ in the sequel.

\subsubsection{No Leverage or Jumps in the Return Generating Process}

The quadratic variation represents the cumulative variability of the continuously evolving return process. As such, it is the natural concept for the realized return variation over $[0, t]$ as argued by ABD (2004) and ABDL (2003). This is particularly transparent in a pure diffusive setting without leverage effects, for which the volatility process $\sigma(t)$ is independent of the innovation process $W(t)$, as it then follows that,

$$
r(t) \mid \sigma\left\{[\sigma(\tau)]_{0 \leq \tau \leq t}\right\} \sim N\left(0, \int_{0}^{t} \sigma^{2}(s) d s\right)
$$

where $\sigma\{\sigma(\tau)\}_{0 \leq \tau \leq t}$ denotes the $\sigma$-field generated by the sample path of $\sigma(\tau)$ for $0 \leq \tau \leq \mathrm{t}$. The integrated variance thus provides a natural measure of the true latent $t$-period return variability.

It is important to keep in mind that the integrated variance term in equation (5) represents the expost or realized return variability. Ex-ante, letting the relevant information set at time u be denoted by $\mathscr{F}(\mathrm{u})$, the corresponding concept of forward looking return variability is given by,

$$
\left.V(t)=E\left[\int_{0}^{t} \sigma^{2}(s) d s\right) \mid \mathscr{F}(0)\right]
$$

Since the volatility process generally is genuinely stochastic, see, e.g., Andersen (1992), the realized or integrated variance equals (ex post) the expected variance, $\mathrm{V}(\mathrm{t})$, plus an innovation term. Consequently, even when the correct model is used to predict future return variability, in accordance with equation (6), 
the standardized returns will be fat-tailed relative to a Gaussian benchmark,

$$
r(t) \cdot V^{-1 / 2}(t)+N(0,1)
$$

On the other hand, returns normalized appropriately by the realized return variability are truly Gaussian,

$$
r(t) \cdot\left[\int_{0}^{t} \sigma^{2}(s) d s\right]^{-1 / 2} \sim \text { i.i.d. } N(0,1) .
$$

This result provides a rationalization for why financial returns normalized by volatility forecasts from standard models exhibit fat tails; see, e.g., Bollerslev (1987), Nelson (1991), Chib, Nardari and Shephard (2002), and Forsberg and Bollerslev (2002). At the same time, the result in (8) is infeasible as it relies on standardization with the true integrated volatility, which generally is latent. Nonetheless, it provides the impetus for the development of precise ex-post measurements of the realized return variability. The basic insight is that, by the consistency result in equation (4), high quality intraday data should facilitate improved measurement of the actual return variability. However, a number of practical complications arise in implementing these ideas.

In principle, we should use all available price and quote observations so as to mimic the limiting operation, $\Delta \rightarrow 0$, as best possible. Meanwhile, the assumption that transaction prices or quotes follow a semi-martingale is blatantly violated at the very finest sampling frequencies where the discrete price grid and bouncing between bid and ask prices implies that recorded price changes are either zero or "large" relative to the expected return variability over small time intervals. The average time between ticks for liquid securities often amounts to just a few seconds. The return volatility over such short intervals is typically an order of magnitude less than the lowest feasible price change as dictated by the price grid. Therefore, we only expect the semi-martingale property to provide a decent approximation over horizons such as one- or five minutes, depending on the price grid, market structure and liquidity. As noted above, these issues are the subject of intense scrutiny within a rapidly expanding literature, see e.g., Aït-Sahalia, Mykland and Zhang (2005), ABDL (2000, 2003), Bandi and Russell (2004a,b), Bollen and Inder (2002), Corsi, Zumbach, Müller and Dacorogna (2001), Hansen and Lunde (2006), Oomen (2005), Zhang, AïtSahalia and Mykland (2005), and Zhou (1996). In this study, we can only use return intervals of one minute or longer due to available S\&P 500 futures data. We end up relying on two minute returns as a compromise between acquiring additional information through more frequent sampling and avoiding 
excessive noise through the accumulation of microstructure distortions. This is the identical frequency settled upon by PV (2004). Nonetheless, we also explicitly invoke a correction for i.i.d. microstructure noise which may remain relevant at this frequency, as argued in, e.g., Hansen and Lunde (2006).

Taken together, the results inspire a practical test of the hypothesis that a return process may be treated as generated by a pure diffusion without leverage effects under minimal auxiliary assumptions. The idea is to construct the realized volatility measures along the lines indicated in equation (3), then substitute this integrated variance estimate into equation (8), and finally test whether the standardized trading period return series is statistically distinguishable from a sequence of i.i.d. draws from a $N(0,1)$ distribution. Of course, this involves a joint hypothesis as any rejection also may arise from estimation errors in the integrated variance measure due to more complex market microstructure effects and/or the finite sample biases induced by the discrete intraday sampling frequency. ${ }^{3}$ Hence, it is important to assess if the theory provides a reasonable guide to the distributional properties of actual return series. We shed additional light on these issues in the simulation based investigations below.

\subsubsection{The Impact of Leverage}

There is compelling evidence that many markets, including those for equity indices, are characterized by a pronounced asymmetric relationship between return and volatility innovations. This is often labeled a "leverage effect" although this asymmetry arguably has little, if anything, to do with the underlying financial leverage; see, e.g., Campbell and Hentschel (1992), Bekaert and Wu (2000), and Bollerslev, Litvinova and Tauchen (2005). In this case, the results from Section 2.1.1 are not valid. A key issue is whether this makes a practical difference for the distribution of standardized returns. A second question is whether it is feasible to restore distributional results for this scenario. We explore these issues here.

When the volatility process, $\sigma(t)$, is correlated (negatively) with the return innovation process, $W(t)$, then knowledge of the daily integrated variance is informative regarding the realization of the daily return innovation. Hence, equations (5) and (8) can no longer hold. Nonetheless, the Dambis-DubinsSchwartz theorem (Dambis, 1965, and Dubins and Schwartz, 1965) ensures that a suitably time-changed continuous martingale is a Brownian Motion. ${ }^{4}$ Hence, appropriately sampled returns will be Gaussian

\footnotetext{
3 The asymptotic (for $\Delta \rightarrow 0$ ) theory in BN-S (2002a) and Andersen, Bollerslev and Meddahi $(2004,2005)$ provides a framework for assessing the latter effect.

${ }^{4}$ Formally, any continuous local martingale (started at the origin), $\mathrm{Y}$, can be decomposed as $\mathrm{Y}=\mathrm{B} \circ \mathrm{Q}$ where B denotes a standard Brownian motion and Q represents the quadratic variation of $Y$, see, e.g., Karatzas and Shrieve (1991), Theorem 4.6. As noted earlier, Zhou (1998) and PV (2004) have implemented these ideas previously, although the former did not explicitly associate this procedure with a strategy to deal with leverage style effects.
} 
even in the leverage case. In particular, without jumps the return series in equation (1) is i.i.d. Gaussian if sampled at equidistant increments of the quadratic variation process. Specifically, for a fixed positive period of "financial" time $\tau^{*}$, we sample the logarithmic price process at times, $0=t_{0}, t_{1}, \ldots, t_{k}, \ldots$, where the calendar time sampling points are defined by

$$
t_{k}=\inf _{t>0}\left([r, r]_{t}-[r, r]_{t_{k-1}}>\tau^{*}\right), \quad k=0,1, \ldots,
$$

so that returns are computed over intervals of identical quadratic variation, $\tau^{*}$. It is natural to calibrate $\tau^{*}$ so that the average calendar period associated with the event-time returns equals one trading day. Note that the associated "event" return horizons may reflect highly variable calendar time lengths. ${ }^{5}$

Denoting the sequence of returns sampled in financial time by

$$
R_{k} \equiv p\left(t_{k}\right)-p\left(t_{k-1}\right), \quad k=0,1,2, \ldots,
$$

the following distributional result holds, even in the case of leverage,

$$
R_{k} / \sqrt{\tau^{*}} \sim \text { i.i.d. } N(0,1), \quad k=0,1,2, \ldots
$$

This result is considerably more general than the distributional result in equation (8), and importantly applies for any continuous martingale. As such, this provides a novel way of gauging the presence and strength of the leverage effect by comparing the properties of returns standardized by realized volatility versus the returns obtained from sampling in financial time. This is a fully nonparametric approach, independent of specific modeling choices for the leverage effect and/or the diffusive volatility component. Of course, there will invariably be some measurement error induced into the procedure, as discussed previously, so the simulation setting in Section 3 is important for gauging the practical implications.

\subsubsection{Intraday Jump Identification}

The preceding sections assume the price process follows a continuous sample path diffusion. However, a

5 The notion of "event" time is related to the Mixture-of-Distributions Hypothesis (MDH) originally proposed by Clark (1973), and further developed by Epps and Epps (1986), Tauchen and Pitts (1983), Andersen (1996), and Andersen and Bollerslev (1997a) among others. However, the MDH explicitly operates with a second observable proxy for market activity, namely the trading process, whereas event time here is defined directly with reference to the price process itself. Our approach is also similar in spirit to the concept of theta-time advocated by Olsen and Associates (see, e.g., Dacorogna et al, 2001). The application of a time-change devise also has important precedents in the statistics literature, see, e.g., Lai and Siegmund (1983) and, recently, Chang and Park (2004). In a different context, Oomen (2006) has recently advocated the use of transaction time sampling in the construction of superior realized volatility estimates. 
priori ruling out jumps is unsatisfactory as discontinuities in the price path is entirely consistent with basic no-arbitrage principles. In fact, in an efficient market setting the release of significant "news" should induce an immediate jump in the price. ${ }^{6}$ Moreover, as previously noted, several recent empirical studies highlight the importance of incorporating jumps in the price process; see, among others, Aït-Sahalia (2002), Andersen, Benzoni and Lund (henceforth ABL) (2002), Bates (2000), Chan and Maheu (2002), Chernov, Gallant, Ghysels and Tauchen (2003), Drost, Nijman and Werker (1998), Eraker (2004), Eraker, Johannes and Polson (2003), Johannes (2004), Maheu and McCurdy (2004), and Pan (2002). ${ }^{7}$

When the jump component is present in the specification (1), the distributional results for the pure diffusion case break down. A potential solution is to seek direct identification of the timing of jumps, so that a jump-adjusted price path can be tested based on the distributional implications for the pure diffusive case. BN-S $(2004,2005)$ provide inspiration that this may be feasible, although their results only speak to the presence of jumps over a given trading period and not to their timing or whether multiple jumps have occurred. Hence, we seek an alternative technique that directly identifies the jump timing.

Inspired by BN-S (2004), define the standardized realized bi-power variation measure,

$$
B V_{t+1}(\Delta) \equiv \mu_{1}^{-2} \sum_{j=2}^{1 / \Delta}\left|r_{t+j \cdot \Delta, \Delta}\right|\left|r_{t+(j-1) \cdot \Delta, \Delta}\right|,
$$

where $\mu_{1} \equiv \sqrt{ }(2 / \pi)$. It follows then under weak regularity conditions that for $\Delta \rightarrow 0$,

$$
B V_{t+1}(\Delta) \rightarrow \int_{t}^{t+1} \sigma^{2}(s) d s
$$

Consequently, the bipower variation (asymptotically) annihilates the contribution of jumps to the quadratic variation and only measures the integrated variance attributable to the diffusive component. Combining equations (4) and (13), the contribution to the quadratic variation process due to jumps may therefore be estimated consistently by the difference between realized volatility and bipower variation. BN-S (2004) derive the joint limiting distribution of the realized volatility and bipower variation under the null hypothesis of a continuos sample path, in turn allowing for the construction of formal statistical tests

\footnotetext{
6 This is also consistent with a recent and rapidly expanding literature documenting almost instantaneous price reactions in response to the release of a number of perfectly timed macroeconomic news announcements; see, e.g., Andersen, Bollerslev, Diebold and Vega $(2003,2005)$ and the many references therein.

7 Earlier studies based on time-invariant jump-diffusions include Merton (1976) and Ball and Torous (1983). 
for significant jumps based on the appropriately scaled difference between the two measures. ${ }^{8}$

We now outline an alternative procedure which focuses directly on individual intraday returns through a uniform decision rule that allows for simultaneous identification of multiple significant jump on each trading day. Specifically, given a realization of the robust-to-jumps bipower variation measure, we consider whether a randomly selected intraday return, $r_{t+\xi \cdot \Delta, \Delta}=\sum_{j=1}^{1 / \Delta} r_{t+j \cdot \Delta, \Delta} \mathbb{I}(\xi=j)$, is subject to a jump, where $\xi$ is an independently drawn index (uniformly distributed) from the set $\{1,2, \ldots, 1 / \Delta\}$ and $r_{t+j \cdot \Delta, \Delta}$ have conditional mean and variance given by $\mathrm{E} r_{t+j \cdot \Delta, \Delta}$ and $V r_{t+j \cdot \Delta, \Delta}$, respectively. This randomization device is useful to express the conditional moments of an arbitrary intraday return in terms of the corresponding conditional trading day moments. For example,

$$
\mathrm{E} r_{t+\xi \cdot \Delta, \Delta}=\mathrm{E} \sum_{j=1}^{1 / \Delta} r_{t+j \cdot \Delta, \Delta} \mathbb{I}(\xi=j)=\sum_{j=1}^{1 / \Delta} \mathrm{E} r_{t+j \cdot \Delta, \Delta} \mathrm{E} \mathbb{I}(\xi=j)=\Delta \cdot \sum_{j=1}^{1 / \Delta} \mathrm{E} r_{t+j \cdot \Delta, \Delta}=\Delta \cdot \mathrm{E} r_{t+1}
$$

where $E r_{t+1}$ denotes the daily mean conditional on the daily integrated variance. Recall that this quantity is typically non-zero under leverage, even if the drift is zero. Likewise, for the conditional (randomized) intraday return variance, given the daily integrated variance, $I V_{t+1}=\int_{t}^{t+1} \sigma^{2}(s) d s$ we obtain,

$$
\begin{aligned}
& V r_{t+\xi \cdot \Delta, \Delta}=V\left[\sum_{j=1}^{1 / \Delta} r_{t+j \cdot \Delta, \Delta} \mathbb{I}(\xi=j)\right]=\mathrm{E}\left[\sum_{j=1}^{1 / \Delta} r_{t+j \cdot \Delta, \Delta} \mathbb{I}(\xi=j)\right]^{2}-\left[\mathrm{E} \sum_{j=1}^{1 / \Delta} r_{t+j \cdot \Delta, \Delta} \mathbb{I}(\xi=j)\right]^{2} \\
& =\sum_{j=1}^{1 / \Delta} \mathrm{E} r_{t+j \cdot \Delta, \Delta}^{2} \mathrm{E} \mathbb{I}(\xi=j)-\left(\Delta \cdot \mathrm{E} r_{t+1}\right)^{2}=\Delta \cdot \sum_{j=1}^{1 / \Delta} \mathrm{E}_{t+j \cdot \Delta, \Delta}^{2}-\left(\Delta \cdot \mathrm{E} r_{t+1}\right)^{2} \\
& =\Delta \cdot \sum_{j=1}^{1 / \Delta} I V_{t+j \cdot \Delta, \Delta}-\left(\Delta \cdot \mathrm{E} r_{t+1}\right)^{2}=\Delta \cdot I V_{t+1}-\left(\Delta \cdot \mathrm{E} r_{t+1}\right)^{2} .
\end{aligned}
$$

These calculations verify that the randomized intraday return $r_{t+\xi \cdot \Delta, \Delta}$ retain the standard relationship between the mean and variance of diffusion increments over small intervals, $\Delta$. In particular, the

8 These insights have inspired the construction of practical jump detection techniques by ABD (2005), BN-S (2005), and Huang and Tauchen (2005), valid under slightly different circumstances. An alternative but related nonparametric continuous record asymptotic jump detection scheme based on the Lévy Law for the modulo of continuity for the sample path of a Brownian Motion has recently been developed in a series of papers by Mancini (2004, 2005a,b). 
appropriately scaled version $\Delta^{-1 / 2} r_{t+\xi \cdot \Delta, \Delta}$ has a vanishing mean,

$$
\lim _{\Delta \rightarrow 0} \mathrm{E}\left[\Delta^{-1 / 2} r_{t+\xi \cdot \Delta, \Delta}\right]=\lim _{\Delta \rightarrow 0} \Delta^{-1 / 2}\left(\Delta \cdot \mathrm{E} r_{t+1}\right)=0
$$

and a variance approaching the integrated variance,

$$
\lim _{\Delta \rightarrow 0} V\left[\Delta^{-1 / 2} r_{t+\xi \cdot \Delta, \Delta}\right]=\lim _{\Delta \rightarrow 0} \Delta^{-1}\left(\Delta \cdot I V_{t+1}-\left(\Delta \cdot \mathrm{E} r_{t+1}\right)^{2}\right)=I V_{t+1}
$$

These results inspire our direct jump test based on individual intraday scaled return realizations. In general, we do not know the distribution of this object but only the first two moments. Asymptotically, however, for very frequent sampling the diffusive null implies that each (scaled) intraday return is approximately Gaussian. If we further assume, for tractability, that volatility is constant within the trading day, we have ${ }^{9}$

$$
\Delta^{-1 / 2} r_{t+\xi \cdot \Delta, \Delta} \quad \sim \quad N\left(0, I V_{t+1}\right) .
$$

We may now formalize our jump detection procedure as follows. First, choose the size $\alpha$ of the jump test at the daily level, and define $\beta=1-(1-\alpha)^{\Delta}$ as the level of the corresponding $(1-\beta)$ confidence interval for a randomly drawn intraday diffusive return distributed approximately $N\left(0, \Delta \cdot B V_{t+1}(\Delta)\right)$. Second, detect possibly multiple intraday jumps $\kappa_{s}(\Delta)$ based on the rule:

$$
\kappa_{s}(\Delta)=r_{t+s \cdot \Delta, \Delta} \cdot \mathbb{I}\left[\left|r_{t+s \cdot \Delta, \Delta}\right|>\Phi_{1-\beta / 2} \cdot \sqrt{\Delta \cdot B V_{t+1}(\Delta)}\right], \quad s=1,2, \ldots, \frac{1}{\Delta},
$$

where $\Phi_{1-\beta / 2}$ refers to the corresponding critical value from the standard normal distribution. Note that this procedure will tend to over-reject the diffusive null hypothesis whenever there is substantial intraday variation in volatility. This therefore suggests a conservative choice of $\alpha$ and hence $\beta$. Nonetheless, we later document satisfactory practical performance in terms of effective power and size with $\alpha=10^{-5}$ for a variety of jump-diffusive specifications endowed with significant time-variation in volatility. In fact, we find that it tends to outperform the existing BN-S procedure in term of identifying days with jumps. ${ }^{10}$

9 The assumption of constant intraday volatility greatly simplify matters, as all scaled intraday returns then are i.i.d. Gaussian with a distribution uniquely determined by the conditional mean and variance given in equations (16) and (17). If volatility varies over the trading day, the scaled intraday returns will be heteroskedastic and the conditional distribution (given the daily integrated variance) is then a normal mixture. We will further investigate this issue in the simulations reported on below.

10 There is no claim of optimality for this procedure, and future research will surely provide improvements. Given the lack of theory for direct identification of jump timing we simply exploit the constant volatility benchmark to develop a feasible approach and then verify that it performs well for relevant models. An alternative approach is provided 
The ability to draw inference regarding the timing and size of jumps allow us to devise a nonparametric strategy for deriving distributional implications within the jump-diffusion setting. First, we subject the intraday return series to a jump identification scheme and remove identified jumps. These returns are then seen as, approximately, generated by a pure diffusion process, so that we can apply the techniques suitable for that case, discussed in the preceding sections. However, before we assess the empirical merit of this approach in more detail, we need to discuss some pertinent implementation issues.

\subsection{Testing for Distributional Features of Jump-Adjusted Standardized Returns}

Under ideal circumstances, including frictionless markets and perfect jump extraction techniques, the jump-adjusted and appropriately standardized trading day returns are asymptotically, for ever finer sampling, distributed as i.i.d. standard normal variables, as stated in equations (8) and (11) for the distinct diffusion cases. This property serves as the main benchmark for our empirical investigation concerning the descriptive validity of the jump-diffusion setting, based upon the actual return distributions calculated from the limited number of intraday trading day returns at our disposal.

\subsubsection{Some General Properties of Standardized Trading Day Returns}

An important fact is that returns standardized by realized volatility will be thin tailed by construction. This follows from the Cauchy-Schwartz inequality. Let $n=1 / \Delta$ denote the number of intraday returns,

$$
\left|\sum_{j=1}^{n} r_{t+j \cdot \Delta, \Delta}\right| \leq \sqrt{\sum_{j=1}^{n} 1^{2}} \sqrt{\sum_{j=1}^{n} r_{t+j \cdot \Delta, \Delta}^{2}}=\sqrt{n} \sqrt{R V_{t+1}(\Delta)}
$$

so that

$$
\left|\sum_{j=1}^{n} r_{t+j \cdot \Delta, \Delta}\right| / \sqrt{R V_{t+1}(\Delta)} \leq \sqrt{n}
$$

Consequently, the distribution of the standardized returns has finite support, or truncated tails, as all realizations fall within the $[-\sqrt{n}, \sqrt{n}]$ interval. This result is valid for all return generating processes, including those with jumps and microstructure noise. Moreover, notice that equality is achieved only when all intraday returns are identical and thus constant throughout the entire trading day. ${ }^{11}$

by the recursive strategy recently pursued in Andersen, Bollerslev, Frederiksen and Nielsen (henceforth ABFN) (2005).

11 We are grateful to Nour Meddahi for this argument which offers a more elegant and simpler reasoning than the discussion provided in an earlier version of the paper. 
Similar reasoning suggests that the presence of jumps renders the standardized returns thin tailed. For notational simplicity, let the set of intraday returns be denoted by $\left(x_{1}, x_{2}, \ldots, x_{n}\right)$, and assume, without loss of generality, that $\sum_{j=1}^{n} x_{j}=c>0, \quad$ so the trading day return is positive. Now, imagine that the largest intraday return, say $x_{i}$, represents a positive jump so it is of the same sign as the daily return as is typical if it is large enough to exert a marked impact on the overall return. Upon taking the partial derivative of the standardized return, $\sum_{j=1}^{n} x_{j} \cdot\left(\sum_{j=1}^{n} x_{j}^{2}\right)^{-1 / 2} \quad$ with respect to $x_{i}$, one may readily determine that the impact is negative if and only if,

$$
x_{i}>\sum_{j=1}^{n} x_{j}^{2} / c
$$

This condition is trivially satisfied under the assumption on the jump since the largest intraday return must exceed the right-hand-side of (22) unless all intraday returns equal $c / n$ and (22) holds as an equality.

These arithmetic arguments support the notion that jumps tend to render the standardized returns thin tailed. However, it is straightforward to construct counterexamples by considering jumps of the opposite sign of the overall return. Similarly, if one jump helps offset another large jump on the same day, the effect is generally unpredictable. Our simulation sections help shed additional light on the issue.

\subsubsection{Finite Sample Results for the Diffusion Case}

For simplicity, we now consider a pure diffusion (equivalently all jumps are perfectly identified and removed) and assume $n$ equidistant intraday return observations are available. We continue to focus on the case of constant diffusive volatility within the trading day to obtain insights within a tractable setting. It then follows from PV (2004) that the density function for the standardized returns,

$$
\tilde{R}_{t+1} \equiv \sum_{j=1}^{n} r_{t+j \cdot \Delta, \Delta} / \sqrt{R V_{t+1}(\Delta)}
$$

takes the explicit form,

$$
f_{\tilde{R}}(\tilde{r})=\frac{\Gamma(n / 2)}{\sqrt{\pi n} \Gamma((n-1) / 2)}\left(1-\frac{\tilde{r}^{2}}{n}\right)^{\frac{(n-3)}{2}} 1_{(-\sqrt{n}, \sqrt{n})}(\tilde{r}), \quad \tilde{r} \in \mathbb{R} .
$$


A number of observations are in order. First, the support of the distribution is $[-\sqrt{n}, \sqrt{n}]$ as for the general case in Section 2.2.1. Hence, the distribution is truncated and has thin tails. Second, equations (8) and (11) imply that the density in (24) converges to the standard normal for $n \rightarrow \infty$. We illustrate this effect in Figure 1 for different values of $n$. The normal approximation is very poor when based on only a few high-frequency observations. For $n \geq 48$ it works reasonably in the center of the distribution, but the tail behavior is only close to that of the normal for even higher values of $n$. Third, if instead the return period is defined through the increment to realized volatility, as described in Section 2.1.2, n, the number of intraday observations may vary widely across “days." If a full (financial) trading period consists of only a handful of intraday observations, some of these are necessarily quite extreme, potentially undermining the usefulness of the constant-within-trading-day volatility assumption. In such instances, the distributional approximation in (24) is likely unreliable, as the simulations later on also confirm.

\subsubsection{Finite Sample Biases and Statistical Tests}

A popular set of normality tests is based on a comparison of higher order theoretical moments with the corresponding sample moments. In the current context, we have the sharp asymptotic i.i.d. $N(0,1)$ null hypothesis for the standardized daily returns rather than a generic $N\left(\mu, \sigma^{2}\right)$ null. Hence, we may compare the third and fourth sample moments directly to their theoretical values without any initial demeaning and scaling of the observed series as is done, for example, when applying the common Jarque and Bera (henceforth JB) (1980) test. In fact, such initial transformations of the data will induce finite sample size distortions in the JB test under the i.i.d. Gaussian null as argued by Bontemps and Meddahi (henceforth BM) (2002). Hence, the focus on the exact null hypothesis is likely to bring about improvements in both the size and power compared to the generic normality tests. Further efficiency gains are feasible via a test based on the joint distribution of the first four moments. BM (2005) argue such a test, implemented through a representation based on the first elements of the Hermite polynomial expansion, brings about a number of important advantages, including robustness towards parameter estimation uncertainty. Below, we implement a GMM style test which is identical to the corresponding BM test for the current setting.

Such normality tests may actually result in an undesirable high level of power, as the finite sample moments inevitably are downward biased, thus producing an excessive rejection rate even if the theory is valid. Specifically, under the assumptions in Section 2.2.2, the fourth moment of the standardized trading day return equals $3 n_{t} /\left(n_{t}+2\right)$. If the number of intraday observations is low, this finite-sample "bias," driving the kurtosis below the asymptotic value of 3, is substantial. In practice, the intraday returns are also unlikely to be i.i.d., so the issue is likely even more pertinent than suggested by this illustration. Such 
considerations motivate the exploration of corresponding moment based tests for the approximate finitesample distribution in equation (24).

In particular, it follows directly from PV (2004) that,

$$
\mathrm{E}\left[\tilde{R}_{t}^{(2 k-1)}\right]=0, \quad \mathrm{E}\left[\tilde{R}_{t}^{2 k}\right]=\frac{n_{t}^{k} \cdot(2 k-1) \cdot(2(k-1)-1) \ldots \cdot 1}{\left(n_{t}+2 k-2\right) \cdot\left(n_{t}+2(k-1)-2\right) \ldots \cdot n_{t}}, \quad k=1,2, \ldots
$$

Denoting the corresponding sample and population moments by,

$$
\bar{M}_{T}^{(k)}=\frac{1}{T} \sum_{t=1}^{T} \tilde{R}_{T, t}^{k} \quad, \quad \text { and, } \quad M_{T}^{(k)}=\mathrm{E}\left[\bar{M}_{T}^{(k)}\right],
$$

respectively, then under standard regularity conditions, as detailed in the appendix,

$$
\begin{aligned}
& T \frac{\left[\bar{M}_{T}^{(1)}\right]^{2}}{1} \sim \chi^{2}(1), \quad T \frac{\left[\bar{M}_{T}^{(2)}-1\right]^{2}}{M_{T}^{(4)}-1} \sim \chi^{2}(1), \quad T \frac{\left[\bar{M}_{T}^{(3)}\right]^{2}}{M_{T}^{(6)}} \sim \chi^{2}(1), \quad T \frac{\left[\bar{M}_{T}^{(4)}-M_{T}^{(4)}\right]^{2}}{M_{T}^{(8)}-\left[M_{T}^{(4)}\right]^{2}} \sim \chi^{2}(1), \\
& T\left[\frac{\left[\bar{M}_{T}^{(3)}\right]^{2}}{M_{T}^{(6)}}+\frac{\left[\bar{M}_{T}^{(4)}-M_{T}^{(4)}\right]^{2}}{M_{T}^{(8)}-\left[M_{T}^{(4)}\right]^{2}}\right] \sim \chi^{2}(2),
\end{aligned}
$$

and

$$
T\left(\bar{M}_{T}^{(1)} \quad \bar{M}_{T}^{(2)}-1 \quad \bar{M}_{T}^{(3)} \quad \bar{M}_{T}^{(4)}-M_{T}^{(4)}\right)\left[\begin{array}{cccc}
1 & 0 & M_{T}^{(4)} & 0 \\
0 & M_{T}^{(4)}-1 & 0 & M_{T}^{(6)}-M_{T}^{(4)} \\
M_{T}^{(4)} & 0 & M_{T}^{(6)} & 0 \\
0 & M_{T}^{(6)}-M_{T}^{(4)} & 0 & M_{T}^{(8)}-\left[M_{T}^{(4)}\right]^{2}
\end{array}\right]^{-1}\left(\begin{array}{ll}
\bar{M}_{T}^{(1)} \\
\bar{M}_{T}^{(2)} & -1 \\
\bar{M}_{T}^{(3)} & \\
\bar{M}_{T}^{(4)}-M_{T}^{(4)}
\end{array}\right) \sim \chi^{2}(4)
$$

These quadratic forms enable tests for the validity of the approximate finite-sample distributional theory in equation (24). We focus on the usual strategy of relying on either individual moments, the joint third and fourth moments, or the joint first four moments. Of course, if the number of intraday observations is uniformly large, i.e., $n_{t} \rightarrow \infty$ for all $t$, the population moments converge to the standard normal values, $M_{T}^{(1)}=M_{T}^{(3)}=0, M_{T}^{(2)}=1, M_{T}^{(4)}=3, M_{T}^{(6)}=15$, and $M_{T}^{(8)}=105$. The results from the finite-sample tests may then be compared directly to the corresponding i.i.d. $N(0,1)$ asymptotic moment tests obtained by substituting the population moments into the quadratic forms above. As shown in the appendix, this GMM normality test based on the first four moments is identical to the BM (2005) test constructed from the first 
four Hermite polynomials. Of course, many alternative tests for i.i.d. $\mathrm{N}(0,1)$ exist and we explore a number of these as well, including some common Empirical Distribution Function (EDF) tests.

\section{Simulation Evidence}

This section provides evidence on the finite sample distribution of jump-adjusted and realized volatility standardized return series. We assume that the underlying high-frequency returns are generated by a jump diffusion calibrated to parameter values obtained through recent empirical studies of the S\&P 500 equity index. The base scenario features pronounced volatility persistence, a strong leverage effect, and fairly frequent jumps. We explore the separate impact of these features by studying a pure diffusion and a jump diffusion, both with and without leverage effects. We also allow for different jump intensities and jump sizes. All experiments are conducted for a range of financial time trading periods, $\tau^{*}$, different return horizons, along with different sample sizes. Finally, we shed light on the effect of i.i.d. microstructure noise, and the limiting behavior of our testing procedure (for an increasing number of trading days) as we simulate series corresponding to much larger data sets than those currently available with real data.

\subsection{A Standard One-Factor Stochastic Volatility Jump-Diffusion Model}

Our simulation evidence is based on the one-factor stochastic volatility jump-diffusion model estimated by ABL (2002) from daily S\&P 500 data, but the specification of the jump component is also influenced by the subsequent study in ABD (2005). The simulations capture the dominant features of the equity index returns, yet the structure is sufficiently simple to allow for direct interpretation of the impact of the various features of the return generating process. Specifically,

$$
\begin{aligned}
& d p(t)=\sigma(t) d W_{1}(t)+\kappa(t) d q_{t} \\
& d \sigma^{2}(t)=\eta\left(\theta-\sigma^{2}(t)\right) d t+v \sigma(t) d W_{2}(t),
\end{aligned}
$$

corresponding to a standard affine (latent) stochastic volatility model augmented by a jump component. If there are no jumps and the two Wiener processes are independent, we have a simple affine diffusion with the three parameters $\theta, \eta$, and $v$ controlling, respectively, the unconditional (daily) return variance, the strength of mean reversion in volatility, and the volatility-of-volatility. Obviously, in this setting equation (8) applies. The complications of primary interest are introduced via correlation between the return and volatility innovations as well as jumps. We capture these features through the following representation, 


$$
d q_{t} \sim P o(\lambda) ; \quad \kappa(t) \sim N\left(0, \widehat{\nabla}^{2}\right) ; \quad \operatorname{corr}\left(d W_{1}(t), d W_{2}(t)\right)=\rho
$$

The Poisson process has constant intensity rate, $\lambda$, with normally distributed jump size, so the jump returns are approximately zero mean and Gaussian. The strength of the leverage effect is governed by $\rho$.

The diffusive parameters are fixed throughout at $(\theta, \eta, v)=(1,0.01,0.1)$ to keep the simulation size manageable. This implies an unconditional daily return variance of $1 \%$ and a mean reversion for daily stock returns which is roughly consistent with ABL (2002). The leverage effect is also, whenever present, fixed to reflect recent empirical evidence from U.S. equity indices, namely $\rho=-0.5$. Finally, the jump parameters are inspired by the contribution of jumps to overall daily return volatility in ABD (2005), although we experiment with different jump intensities and sizes to gauge the impact of this critical component for the properties of the standardized return series.

We provide results for a simulated sample size of 5,000 'days' with 195 'intraday' return observations, corresponding to the use of two-minute returns over a $6 \frac{1}{2} 2$ hour trading day, reflecting our actual implementation with the S\&P 500 futures data in Section 4. We approximate the diffusion process through an Euler scheme and sample once every 'second' in order to achieve a high degree of precision, although the actual prices are only recorded every 120 seconds. We produce a total of 1,000 simulated samples for each scenario, and we vary the length of the trading period over which we construct the standardized trading day returns from $1 / 2$ 'day' up to $2 \frac{1}{2}$ 'days' in increments of $1 / 2$ 'day,' although we only report findings for $1 \frac{1}{2}, 1$ and $2 \frac{1}{2}$ 'days' for brevity. These are all based on the original 5,000 trading days, so there are 10,000 half and 2,000 biweekly trading periods. We fix the number of trading days to not exploit more data in the simulation than is available in practice - intraday data typically are not available before 1985, leaving a maximum of about twenty years of data. This configuration implies a trade-off in the choice of a longer versus shorter trading period return. Shorter trading period ( $1 \frac{2}{2}$ day) utilizes fewer intraday returns so they are relatively more noisy but there are more trading periods available over the 5,000 trading day sample, so the sampling variation can be assessed better than for the longer trading period ( $2 \frac{1}{2}$ day). However, the simulations can, of course, transcend such limitations. We explore the limiting behavior of our tests by simulating corresponding long samples consisting of 50,000 trading days.

For every simulated return series we construct the corresponding jump-adjusted intraday series and aggregate to different horizons to obtain the jump-adjusted trading period returns. We calibrate the jump detection procedure to obtain a $0.001 \%$ chance of falsely identifying a jump on a given trading day (conditional on constant volatility within the day). Hence, we employ a conservative jump extraction 
technique which only will flag price moves that we are quite certain represent actual discontinuities. Of course, with time-varying volatility the actual significance level is somewhat higher as illustrated below. Finally, both the original (physical) intraday series and the jump-adjusted series is converted into financial period return series. These financial time return observations cover a varying (calendar) time interval, but we calibrate the financial time clock via the choice of $\tau^{*}$ to obtain separate series which cover an average trading period matching those for the physical return series.

\subsection{Simulation Results}

For each simulation scenario we provide a variety of distributional tests conveying information about the quality of fit. The most basic information is conveyed by the descriptive statistics for the standardized returns, various direct i.i.d. $\mathrm{N}(0,1)$ test sizes and the size of moment tests inspired by the approximating distribution in (24). We include three empirical distribution function (EDF) tests for i.i.d. N(0,1), namely the Kolmogorov-Smirnov (KS) test, the Anderson-Darling (AD) test and the Cramer-von Mises (CVM) test. The former is sensitive to deviation between the empirical distribution and the standard normal over the entire support, while the latter two pay more attention to the tail behavior. We also explore the performance of the generic JB test for normality based on the third and fourth sample moments. As explained, this test is incorrectly sized but we included it due to its popularity in the wider literature.

\subsubsection{Evidence on the Intraday Jump-Detection Procedure}

For each simulation design we implement our jump extraction procedure detailed in Section 2.1.3. The jump test is conducted at a (daily) nominal size of $0.001 \%$, but the actual size will be slightly higher in realistic settings with time-varying intraday volatility. This section compares our intraday jump detection technique with the prevailing BN-S technique based on the discrepancy between the realized volatility and bipower variation over each "trading day." We implement the ratio version of their test with maximum adjustment. This approach is the preferred test among various candidates in Huang and Tauchen (2005).

The top panel of Table I reports on the BN-S jump test with a size of $0.1 \%$. Simulation designs \#1-4 have no leverage effect, but varying jump intensity and size parameters, while simulation designs \#5$8 \mathrm{n}$ are similar except for the presence of a non-zero leverage coefficient, $\rho=-0.5$. We discuss the simulation findings for the various tests in detail in the following sections, focusing in this section exclusively on aspects of the jump detection procedures. Moreover, since the presence of leverage effects appear to have minimal impact on the performance, we focus our discussion on the first four designs.

The first row in the table corresponds to the case of no jumps. The BN-S test is seen to be 
somewhat oversized at $0.16 \%$, but the average daily imputed jump volatility (Det JV) is zero to three decimals, reflecting the fact that the (falsely) identified jumps are quite small, and the associated root mean squared error (RMSE) is also minimal at $0.009 \%$. Nonetheless, the performance is inferior to that of our intraday based procedure, as indicated in the middle panel of Table I. Here, the RMSE is $0.005 \%$. This procedure also provides a slight improvement in size. Finally, the intraday procedure with size $0.001 \%$ produces RMSE of $0.001 \%$, as indicated in the lower panel of the table. Hence, the errors stemming from falsely detected jumps are minimal in this scenario. Moreover, the lower size results in only a slight loss of power. This is evident from the last column, where the power reflects the percentage of days with actual jumps that are detected by the test. Furthermore, this version of our procedure still retains more power than the larger sized BN-S test. Even more significantly, our intraday jump detection approach with minimal (theoretical) size of $0.001 \%$ still produces root mean squared errors across all designs that are substantially lower than for the BN-S procedure. This suggests that our test uniformly dominates the BN-S test. Our suspicion that the actual data display somewhat more irregular features than captured by our simulation design motivates our conservative choice of test size $0.001 \%$ relative to $0.1 \%$, even if the evidence in Table I, if anything, indicates slightly better performance of the latter. Finally, we note that the relative size of the RMSE due to true jump volatility (True JV) is largest in the "moderate jump scenario" (\#4) compared with the "rare large jump" (\#2) and the "frequent small jump" (\#3) designs. As this case may be more representative of the actual data we pay particular attention to this scenario below.

\subsubsection{The No-Leverage Pure Diffusion Case}

The most basic scenario involves the pure diffusive process with no correlation between the return and volatility innovations. In this setting stochastic volatility is effectively a pure time deformation device. If we control for the integrated volatility through the corresponding realized volatility measures, we should recover standard normality (approximately) as stated in equation (8). Of course, in practice we do not know the properties of the data generation process so we also explore what happens if we transform the series into financial time and/or implement initial jump detection and extraction procedures.

The first set of simulations are obtained from model (29) with the extraneous parameters from equation (30) zeroed out. The descriptive statistics for the standardized return series are given in Table II.1A, top panel, for design \#1. As expected, the averages of the mean, standard deviation and skewness equal the expected values for the standard normal distribution. ${ }^{12}$ In contrast, there is a downward bias in

\footnotetext{
12 For brevity, we do not report the standard deviation across the simulated samples. It is relatively small in each design and for all summary statistics. They were reported in the initial working paper draft of the paper.
} 
the kurtosis, even compared to the expected value which accounts for the impact of the finite sample reported in the last column (assuming constant intraday volatility). The bias is most pronounced for the short trading period and almost vanishes at the biweekly frequency. Only for this longer period does the fourth moment begin to match the expected value, $3 n_{t} /\left(n_{t}+2\right)$. These observations underscore the potential importance of developing the moment (finite sample) adjusted test statistics below.

We provide evidence on the size of some common normality tests in Table II.2A. For parsimony, we report results for test size $5 \%$ only, but qualitatively identical results obtain for other significance levels. The EDF tests KS, CVM and AD all have good size properties, albeit with a tendency towards overrejection. This is likely associated with the mild deviations from standard normality in finite samples discussed previously. The JB tests behave more erratically. As expected, they over-reject for short trading periods where the bias in the kurtosis is most pronounced. However, the JB tests are undersized at daily and longer frequencies due to initial centering and scaling, so they under-reject even if the (finite sample) distributions are not exactly $\mathrm{N}(0,1)$. If one follows PV (2004) in adjusting the kurtosis in the JB test to equal the finite sample adjusted value, the over-rejections for the half-day period are reversed to underrejections. Moreover, these under-rejections amplify for the longer trading period returns. The jump detection step has no discernable impact on the results, whereas (unwarranted) conversion into financial time is a bit more problematic as the tests now appear to be systematically, albeit mildly oversized.

A more direct testing strategy for the standardized returns is to exploit the moments of the two approximating distributions, namely the standard normal and the finite sample approximation in equation (24), thus sidestepping the loss of power associated with the JB test. Such moment based results are presented in Tables II.3A and II.4A for each individual moment and selected combination of the moments, including a joint test based on all four moments. Table II.3A refers to the test size computed against the standard normal moments. The size of the tests based on individual moments appear sensible, while the joint moment tests are oversized for the smallest trading period. However, this problem vanishes as the trading period is increased. This is, of course, not surprising since the normal approximation is found to be quite accurate at the daily trading frequency and above in Figure 1. The results in Table II.4A based on the approximating finite sample distribution are uniformly impressive, as they also account for the downward bias in the fourth moment over the lowest trading periods. These results also suggest that the convergence of the sampling distributions to the standard normal becomes reliable around the one trading day period. Consequently, these tests provide a convenient supplement to the empirical distribution tests for standard 
normality, whereas the JB tests are much less reliable. ${ }^{13}$

\subsubsection{The No-Leverage Jump-Diffusion Case}

The impact of jumps and the difficulty in identifying and adjusting for them likely hinge on specific features of the jump process. We explore two benchmark cases and one in-between. For the first scenario, the parameters vector is $\left(\vartheta^{2}, \lambda\right)=(2.5,0.1)$. This induces a jump about once every two weeks and the jumps account for $20 \%$ of overall return volatility which is consistent with the evidence in ABD (2005), even if the jump sizes may appear slightly exaggerated. As such, this scenario should provide a good sense for how the presence of relatively large jumps impacts the distribution of the standardized returns.

Turning to the results, we note from Table II.1A that the kurtosis for the standardized return series unadjusted for jumps now is more downward biased. This is in line with the reasoning in Section 2.2.1 that jumps induce thin tails. In contrast, there is essentially no bias for the jump-adjusted series relative to the finite sample corrected values, reflecting the success of the test in capturing the large jumps. These encouraging findings are confirmed in Table II.2A where the empirical distribution function (EDF) tests for the jump-adjusted series all are correctly sized for the one day trading period and beyond, although a slight deterioration occurs for the financial time transformed series, as noted before. Hence, in the absence of leverage, this transformation is moderately harmful. Not surprisingly, we now find severe size distortions for the series unadjusted for jumps. These conclusions are collaborated by the moment tests. The test based on all four moments is oversized for all scenarios except the jump-adjusted series with trading periods beyond the $1 / 2$ day in Table II.3A, while the joint adjusted moment tests in Table II.4A provide the same inference except that the jump-adjusted series for the $1 / 2$ trading day now also is correctly sized. This is, of course, consistent with the bias adjustment being more important at this return frequency.

Overall the results suggest that our procedure for jump adjustment, financial time transformation, and standardization provides a feasible method for constructing correctly sized tests for the distributional properties of a diffusion extended with rare large jumps. Of course, these findings may depend on the specific scenario in which, as seen from the bottom panel of Table I (set \#2), our jump detection scheme performs fairly well. The extracted jumps account for $24.9 \%$ of return variability compared to the true contribution of $25 \%$, and we correctly identify $82 \%$ of the jump days. We clearly identify the majority of the large jumps, even if there are mistakes in inferring the exact size of each jump. We next check if these

\footnotetext{
13 We also explored the size properties of standard Ljung-Box tests for uncorrelated standardized return and squared return series. These test statistics are uniformly well-behaved, reflecting the fact that the returns are uncorrelated and standardization with realized volatility effectively removes the systematic persistent volatility component. Thus, the squared standardized returns are also effectively uncorrelated for all designs. These results are available upon request.
} 
findings carry over to settings where jumps are harder to identify.

We now consider frequent but small jumps (set \#3), with the same total contribution to volatility. Specifically $\left(\vartheta^{2}, \lambda\right)=(0.25,1)$, so that jumps are about ten times more frequent, but also ten times smaller. If anything the results are stronger, in terms of illustrating the importance of jump adjustments and achieving satisfactory size for the jump-adjusted standardized return series in Tables II.3A and II.4A.

Finally, we explore a "moderate" jump scenario $(\operatorname{set} \# 4)\left(\vartheta^{2}, \lambda\right)=(0.5,0.2)$, with a smaller overall jump contribution of $10 \%$ to return variability. The bottom panel of Table I shows that the tests now miss a substantial fraction of the jumps, but still identify most large jumps, even if the errors are somewhat larger than before. Even if the tests for the jump-adjusted series are appropriately sized, it is now harder to distinguish the findings for the jump-adjustment series from those of the unadjusted returns. In fact, the rejection rates for the unadjusted series at trading periods of one day or more in Tables II.2A-II.4A are never much above $10 \%$ for the 5\% test level. In contrast, the EDF tests are comparatively well behaved and provide at least as much discriminatory power as the moment tests.

\subsubsection{The Pure Diffusion with Leverage Case}

We now exclude jumps but introduce a strong, albeit realistic, leverage effect by letting $\rho=-0.5$. Table II.1B (set \#5) reveals that leverage has a very different impact than jumps. Now, the mean is biased upward whereas the kurtosis (unadjusted for the non-zero mean) accords with the theoretical value after controlling for the finite number of observations used in the computation of the trading period returns. The upward shift in the mean is due to the stronger standardization of negative returns stemming from their correlation with the volatility innovations and hence realized volatility. Table II.2B shows that the EDF tests are substantially oversized for all (physical) calendar period return series, whether jump adjusted or not, while they are well sized for the returns computed in financial time. Hence, the financial time transformation is successful, as stipulated by equation (11). These findings are further collaborated by Tables II.3B and II.4B. Another distinct difference to the jump scenario is the lack of problems with test size even for the shorter financial time periods, so finite sample considerations are simply less pertinent in this situation. The realized volatility measures approximate the underlying integrated variances sufficiently well, so that the transformed series are remarkably close to standard normal.

\subsubsection{The Jump-Diffusion with Leverage Case}

Finally, we combine the leverage effect with the jump scenarios explored in Section 3.2.3. This produce the setting arguably most reminiscent of the actual equity index return series. The results are documented 
in Tables II.1B-II.4B (set \#6-8). The descriptive statistics now indicate both an upward bias in the mean and a downward bias in the kurtosis, as may be expected from a combination of the above scenarios. When the jump contribution to overall return variability is high (set \#6 and \#7) it is critical to perform both a jump adjustment and a financial time transformation in order to obtain correctly sized tests. The two features appear to reinforce each other to produce quite badly sized tests if either of the steps are omitted. For the moderate jump scenario (set \#8), the same qualitative findings obtain in attenuated form. This suggests that within the moderate jump scenario the financial time transformation is by far the most critical step in terms of achieving reasonably sized tests.

\subsubsection{Larger Sample Size}

The power to reject normality increases with sample size, so our procedure for restoring normality should stand out even more clearly on larger samples. We simulate 1,000 sets of 50,000 days for the moderate scenario with leverage and jumps, which represents the hardest challenge for identifying incorrectly sized tests within our initial simulation battery of 1,000 x 5,000 days. We report the findings in the bottom panel of Tables II.1B-II.4B (set \#X8). The results are striking as the power to reject normality for incorrectly standardized returns approaches $100 \%$ in many cases, even though our three-stage standardization procedure remains approximately correctly sized. Moreover, notice that the large sample size enhances our ability to detect the impact of the finite intraday observation distortions. In Table II.3B, the joint four moment test for normality of the financial time transformed and jump-adjusted series reject $99.8 \%$ of the times for the $1 / 2$ day returns, while the corresponding number in Table II.4B is an appropriate $5.2 \%$. Nonetheless, it is evident that it is very hard to obtain high power in terms of detecting the specific features of the jump-diffusive process in this moderate jump and leverage design. For daily sampling, the adjusted joint moment tests in Table II.4B never reject more than $40 \%$ of the time. It is notably easier to detect the finite sample (in terms of intraday returns per day) deviation from normality than it is to ascertain the presence of the leverage and jump features from the appropriately adjusted moments. Of course, this jump scenario is deliberately chosen to present a challenge. Actual data are likely to display a higher jump intensity and have jumps that routinely exceed the ones simulated here. Hence, it is tempting to deem strong rejections of the null hypothesis based on our adjusted moment test to provide compelling evidence against the jump-diffusive representation. However, this does not address another issue which invariably impacts any test based on actual return data, namely market microstructure frictions or noise.

\subsubsection{Microstructure Noise}


The results in Tables II.1-II.4 indicate that our three-stage methodology works quite satisfactorily for the simulated scenarios. However, this is an idealized setting void of market microstructure complications. It is outside the scope of the paper to explore this topic in depth, so we only consider the simplest and reasonably descriptive case of i.i.d. noise in the price observations. This case has been analyzed quite extensively in the literature starting with Zhou (1996). We begin by briefly setting the stage and describing the motivation for our specific simulation design.

Under the i.i.d. noise hypothesis the logarithmic prices are observed with error, resulting in the following returns,

$$
\hat{r}_{t+j \cdot \Delta, \Delta}=\left(p_{t+j \cdot \Delta}-p_{t+(j-1) \cdot \Delta}\right)+\left(\eta_{t+j \cdot \Delta}-\eta_{t+(j-1) \cdot \Delta}\right)=r_{t+j \cdot \Delta, \Delta}+\left(\eta_{t+j \cdot \Delta}-\eta_{t+(j-1) \cdot \Delta}\right)
$$

where the "true" return series, $r_{t+j \cdot \Delta, \Delta}$ is assumed to be independent of the i.i.d. error sequence, $\eta_{t+j \cdot 4}$, The random price error induces excess variation in the realized volatility and negative serial correlation in the high-frequency returns. In particular, for $\eta_{t}$ i.i.d. $\left(0, \omega^{2}\right)$, it follows readily that,

$$
E\left[\sum_{j=1}^{n} \hat{r}_{t+j \cdot \Delta, \Delta}^{2}\right]=[r, r]_{t+1}-[r, r]_{t}+2 \cdot n \cdot \omega^{2} \equiv Q V_{t+1}+2 \cdot n \cdot \omega^{2}
$$

and

$$
E\left[\sum_{j=0}^{n} \hat{r}_{t+j \cdot \Delta, \Delta} \cdot \hat{r}_{t+(j-1) \cdot \Delta, \Delta}\right]=-n \cdot \omega^{2}
$$

Equation (32) shows that the i.i.d. error structure induces excess variation that cumulates proportionally with the sampling frequency. Consequently, the presence of noise renders realized volatility biased and inconsistent (as $\Delta \rightarrow 0$ ) for the underlying quadratic variation, $\mathrm{QV}_{\mathrm{t}+1}$. However, equation (33) immediately suggests a simple bias correction, as the unknown noise parameter $\omega^{2}$ may be estimated directly from the (negative) first order sample return auto-covariance. The procedure for bias correction in financial time is only slightly more complex. Instead of accumulating squared return innovations until the target, $\tau^{*}$, is surpassed, the modified stopping rule simply becomes,

$$
t_{k}=t_{k-1}+\Delta \cdot \underset{s=1,2, \ldots}{\operatorname{argmin}}\left(R V_{t_{k-1}+s \cdot \Delta}-R V_{t_{k-1}}-2 s \hat{\omega}^{2}>\tau^{*}\right), \quad k=1,2 \ldots
$$


From equation (32), it is evident that the (daily) quadratic variation is invariant to the sampling frequency. In contrast, the second noise (bias) term is intimately tied to each individual price observation and its size is a direct function of the sampling frequency. Hence, to convey the strength of the market microstructure friction in a manner which is independent of sampling frequency, it is standard to report the (average) noise-to-signal ratio for a specific market as $\lambda=\omega^{2} / I V$, where $I V$ represents an estimate of the average daily (diffusive) quadratic return variation over the full sample period.

For the simulation designs we calibrated the i.i.d. noise to estimates extracted from the S\&P 500 futures index returns. The procedure used for obtaining these estimates is described in Section 4, where we report estimates around $5 \cdot 10^{-5}$ or slightly higher. Thus, to explore the impact of a significant, yet realistic, level of contamination we fix the noise-to-signal ratio across all designs at the comparatively high value of $1 \cdot 10^{-4}$. To conserve space, we focus on the most problematic scenario explored above, namely set \#8 with leverage and a moderate jump regime. We provide results both for series based on return standardization ignoring the presence of jumps and for series where we explicitly correct for the bias as described around equation (34). The basic findings, ignoring the presence of microstructure noise, are reported in Table III.1. The label "\#8n" reflects the fact that this design replicates the scenario used to generate set \#8 in Tables II.1B-II.4B, except for the inclusion of the i.i.d. noise component.

The descriptive statistics in panel one of Table III.1 reveal that the standard deviation of the standardized returns no longer equals unity, but instead is downward biased to 0.97 and 0.98 for the calendar and financial time series, respectively. The noise-induced bias of the realized volatility measures implies that we are inflating volatility and therefore "over-standardizing" the trading day returns. This will render the non-standard Gaussian features of the standardized returns more pronounced and should result in higher rejection rates. The next two panels with the direct normality tests confirm this conjecture. Even for the jump adjusted financial time standardized returns, the power for the joint moment tests increases quite dramatically between Tables III.1 and II.3B. In short, the finite sample distortions and the noise combine to produce significant evidence against standard normality. This interpretation is also confirmed in the bottom panel of Table III.1, where we see that the finite-sample adjusted moment tests for the jumpadjusted financial time returns now are badly oversized, or alternatively have considerable power due to the impact of the noise and the resulting failure of the null hypothesis.

Of course, if one correctly assumes that i.i.d. noise is present and implements a bias correction by estimating $\omega^{2}$ over the full (simulated) sample, it should be possible to alleviate the impact of the noise. Indeed, comparing the results for the bias corrected statistics in Table III.2 with the corresponding entries in Tables II.1B-II.4B confirms that the findings are quantitatively close everywhere, although not 
identical. It is, of course, not feasible to literally undo the noise. The bias correction merely seeks to remove the systematic impact and thus allow for meaningful inference.

This raises the question whether the ability to identify jumps is compromised severely by the presence of noise. From Table II.4B, we know that it is hard to distinguish financial time sampled returns before and after jump adjustment, and we have low power to reject the null of no jumps in the moderate jump scenario with 5,000 daily observations. However, we also found that the power to detect jumps is dramatically improved for the longer sample of 50,000 observations (set \#X8). One may worry that this power improvement is more elusive in the presence of noise. Table III.3 speaks to this issue, by repeating the long sample but now with i.i.d. noise in the return process and with appropriate bias correction (if not, the power to reject is uniformly close to $100 \%$ ). The results are striking as the power to reject the null hypothesis is even higher than before. This occurs because the noise adjustments necessarily is less than perfect, so there is now an additional source of error in the system on top of the finite sample biases. With the very large sample size, the power to reject such confounding sources of non-normality is high. Notice, moreover, that this result is obtained without any serious size distortion for the jump-adjusted financial time sampled returns, as they retain rejection frequencies distributed tightly around the nominal $5 \%$ level. In short, given sufficient data, our sequential adjustment procedure appears to have the ability to identify the qualitative features of the return process even in challenging situations. Of course, for realistic sample sizes it is not always feasible to detect, say, infrequent and moderately sized jumps. However, simple i.i.d. noise does not seem to directly impact our discriminatory power in this regard, as long as the potential presence of noise is recognized and adjusted for. It remains an open question whether these basic findings carry over to more complex noise structures and associated identification and adjustment procedures.

\section{Analysis of the S\&P 500 Futures Returns}

This section explores two-minute transaction returns from the S\&P 500 futures contract traded at the Chicago Mercantile Exchange (CME) from January 1, 1988 through July 26, 2004. ${ }^{14}$ An initial inspection of the high-frequency data revealed a number of problematic days with missing activity, or data errors at the beginning or the end of the active trading day from 9:30am to 4:00pm. To avoid any confounding influences from these problem, we simply eliminated 44 trading days which started or ended with ten consecutive zero returns, leaving us with a total of 4,126 complete trading days. Additional details

\footnotetext{
14 The same basic data up until August 2001 were used by PV (2004), allowing for a direct comparison with their earlier findings. Importantly, the extended sample also allows us to assess the robustness of our findings with respect to the highly volatile aftermath following September 11, 2001.
} 
concerning the construction of the 195 two-minute returns for each of these days is given in the appendix.

\subsection{Testing Standard Normality of Adjusted and Standardized S\&P 500 Returns}

The descriptive statistics in the top panel of Table IV.1 reveal that the mean standardized returns are positive and highly significant, whereas the corresponding financial time mean returns are approximately zero. This strong discrepancy across the sampling schemes is indicative of a pronounced leverage effect as discussed previously. A positive bias is also evident in the skewness, while the standard deviation and kurtosis are strongly downward biased.

Turning to the formal hypothesis tests, the second panel of Table IV.1 provides the EDF tests. The evidence is striking. All EDF tests reject the standard normal null hypothesis with p-values of 0-1\%. In sharp contrast, none of the EDF tests for the series sampled in financial time reject at the $5 \%$ level. The erratic behavior of the JB tests, albeit consistent with our prediction of them being severely undersized for the longer trading period returns, supports the contention that they are largely useless in this context. Moving on to the formal moment based tests, the third panel of the table underscores the strong rejections of the standard normal hypothesis in physical time with p-values uniformly close to zero. Again, the financial time return series appear much closer to the strict Gaussian benchmark, although there are a number of small p-values for the shorter trading periods and especially for the series unadjusted for jumps. This rejection pattern may reflect the inadequacy of the standard normal approximation for the lower number of intraday observations, or it may reflect the lack of power to reject the null as the number of return observations underlying the tests diminish for the longer trading period. The bottom panel of Table IV.1 sheds additional light on the issue by exploiting the finite sample correction derived from the approximating density in equation (24). The calendar time based return series continue to produce extraordinarily strong rejections across the board, while the financial time return series now appear even closer to the standard normal benchmark. There is also some indication that the jump-adjusted series provides the closest approximation to the finite sample corrected moments, as none of the corresponding tests reject at the $10 \%$ level for the one (financial) day returns, while the corresponding unadjusted return series encounter rejections at the 5\% level for some tests and produces a p-value of $7 \%$ for the joint four moment test. As for the previous panel, once we move beyond one trading day none of the tests reject.

A couple of factors may explain our findings. First, we have lower power for the longer horizon returns because of the lower number of time series observations so the similar properties of the jump- 
adjusted and unadjusted series may be relatively uninformative. ${ }^{15}$ Second, if the actual data bear some resemblance to the moderate jump design in our simulation experiments (set \#8), the evidence for 5,000 trading days suggest it may be infeasible to discriminate the pure diffusion from the jump scenario through the distributional properties of the standardized returns alone. Obviously, these points may reinforce each other, thus rendering a clear verdict difficult. Even so, it is striking that the standard normal approximation is acceptable across many dimensions once we transform the return series to financial time.

A more direct assessment of the evidence for jumps in the intraday return series may be obtained from inspection and analysis of the extracted jump series. Our jump identification procedure indicates the presence of 382 jumps, whereas we would expect to detect zero or one jump at the $0.001 \%$ level over the full sample (even taking into account the slightly higher effective size). In total, the jumps contribute about $4.4 \%$ to the overall return variability. The extracted jump series is displayed in Figure II along with the original intraday return series and the jump-adjusted series. It is evident that the intraday return series contain extreme outliers that appear incompatible with a pure diffusive framework. The only remaining visible outliers in the jump-adjusted series occur during volatile periods where they are insignificant at the conservative test level we have adopted. Generally, outliers in realized volatility will induce inliers in the corresponding standardized trading day return series, which are much harder to identify in a small sample. Consequently, it is not surprising that relatively infrequent and small jumps can be more difficult to discern from the standardized returns than from the raw returns themselves.

A couple of issues are worth further discussion. First, we identify relatively few jumps compared with recent studies that implement less conservative jump tests such as ABD (2005). We have confirmed that this remains true even if we increase the test size of our jump detection scheme. The primary reason is the exclusion of the generally less active morning period covering 8:20-9:30am. The main macroeconomic announcement releases take place at 8:30am, so we miss the corresponding, often large, jumps. On the other hand, this period is usually very quiet in the absence of such news, so it is problematic to include it for every trading day, as the distributional properties of financial time and jump-adjusted return series are very sensitive to the number of (active) intraday returns and the requisite finite sample adjustments are dependent on the market being "open" over the periods included in the return computations. In order to avoid problems stemming from such issues, we exclude this heterogeneous segment of the trading day. This is appropriate for testing the distributional predictions of interest here, and it facilitates comparison to the PV (2004) study. In contrast, if the properties of the jump series per se is of primary concern, it may

\footnotetext{
15 Note that PV (2004) only report results for the $2 \frac{1}{2}$ financial day return series.
} 
be advisable to use a lower significance level in the jump tests and to include the periods surrounding the macroeconomics news releases in the analysis. Second, it is intuitively clear that the actual data are a bit more complex than the artificial returns generated in the simulations. The ubiquitous microstructure effects generate an extra layer of noise and both multiple volatility factors and more complex jump processes are likely present. Added variability in the actual return process tends to inflate the realized volatility measures, making it still harder to identify smaller jumps, especially during volatile periods.

The significant positive aspect of our findings is that we cannot reject the basic distributional implications of the generic jump-diffusion model in spite of various possible shortcomings. In fact, in light of the simulation evidence we have assembled compelling evidence for a pronounced leverage effect. Moreover, the case for discontinuities in the high-frequency return series is overwhelming, even if the associated adjustments have a limited impact on the distribution of standardized trading day returns. The theoretical observations regarding the effect of jump removal and the extensive simulation evidence help us understand the potential lack of power that our nonparametric distributional based test procedure may possess versus a no-jump alternative. Of course, as previously noted, the S\&P 500 series analyzed here is artificially low on jumps, and most other relevant asset price series will be subject to both larger and more frequent jumps, rendering the jump detection step even more critical.

\subsection{Results for Noise and Intraday Volatility Adjusted S\&P 500 Futures Data}

The recent study by Hansen and Lunde (2006) offers a comprehensive discussion of the impact of market microstructure noise for realized volatility estimation, along with empirical evidence indicative of more complex noise structures for individual equity returns than the simple i.i.d. process entertained in Section 3.2.7. Nonetheless, they also present encouraging results for procedures that incorporate simple first order auto-correlation type noise adjustments. In this section we explore whether such adjustments have any impact on the findings for the S\&P 500 equity index return series.

Direct estimation of the noise variance based on the expressions in equations (32) and (33) encounters practical problems, as the full sample of 2-minute returns results in a positive first-order autocorrelation. Meanwhile, since jumps are not contemplated in existing theoretical work dealing with microstructure noise, ${ }^{16}$ it seems advisable to omit the most volatile days, where large jumps typically are to be found, from the sample used in estimating the noise variance. Indeed, doing so results in a more robust and, importantly, negative estimate. Of course, simply excluding the most volatile days may impart

${ }^{16} \mathrm{ABFN}$ (2006) also conjecture that the presence of jumps will complicate the estimation of the noise process. 
a negative bias to the inferred average realized (diffusive) volatility, so to counterbalance this we exclude a matching number of low volatility days. Letting $\boldsymbol{Q}$ denote the number of high and low volatility days excluded from the sample, we find the striking pattern in the sample autocorrelation coefficients and associated noise variance estimates depicted in Figures III.1 and III.2. As seen from these figures, increasing the value of $\boldsymbol{Q}$, the serial correlation coefficient stabilizes in the range of -0.010 to -0.013 , while the noise variance reaches a level of about $4 \cdot 10^{-9}$. Moreover, the average daily realized volatility for the jump adjusted return series is consistently above $8 \cdot 10^{-5}$, implying a noise-to-signal ratio, $\lambda$, of approximately $5 \cdot 10^{-5}$. Of course, in the presence of undetected jumps the true value of the diffusive volatility may be slightly lower and the noise-to-signal ratio correspondingly higher. ${ }^{17}$

The findings reported in Table IV.2, obtained from the full sample, but with noise correction estimates based on $\boldsymbol{Q}=500$, convey largely the same message as for the unadjusted series in Table IV.1. However, there are indications that the insights from the simulation study discussed in Section 3.2.7 also are of relevance for the S\&P 500 market. First, we obtain significantly larger p-values at the $1 / 2$ day return horizon for the joint moment tests of the jump-adjusted financial time returns in both the third and fourth panel of Table IV.2 relative to Table IV.1. Given the properties of the data, these are clearly the most relevant entries in the tables. Second, there is a tendency for the distinction between the financial time jump adjusted and unadjusted series in panels three and four to now appear more significant. Whether this truly is indicative of power gains achieved through the control for i.i.d. type noise can only be answered definitively through more extensive empirical work across a number of alternative asset return series.

Another important feature of high-frequency asset prices concerns the strong pattern observed in the level of volatility across the trading day. In particular, it is well established that for markets with a well-defined trading day volatility tends to be highest around the open and close of the market, see, e.g., Harris (1986) and Wood, McInish and Ord (1985). The simulation evidence in Section 3 and the empirical results for the S\&P 500 reported so far ignore such patterns. Meanwhile, as shown by Andersen and Bollerslev (1997b), a failure to properly account for this strong intraday periodicity in volatility may seriously distort any inference regarding the underlying dynamic dependencies in the returns. Hence, as a final robustness check, we compute our summary statistics and distributional test statistics for the S\&P 500 data after we first standardize each of the 2-minute returns with the average sample standard deviation for that particular 2-minute interval of the day. The results are reported in Table IV.3. It is evident that the results closely mirror the ones in Table IV.2, and if anything provides even stronger evidence. The p-

\footnotetext{
17 These considerations also inspired our use of $1 \cdot 10^{-4}$ in the simulation design as a reasonable upper bound on the level of the noise-to-signal ratio in the actual equity index returns.
} 
values for the EDF tests increase slightly and the p-values for the joint four moment tests covering the shorter return horizons also tend to go up after the modified jump adjustments.

One message of this section is that corrections for market microstructure noise may be critical for inference regarding the distributional properties of daily returns obtained from high-frequency data. Moreover, the observed effects for the S\&P 500 are generally consistent with our simulation experiments based on i.i.d. noise. On the other hand, the intraday volatility pattern do not impact our basic conclusions. As such, our supportive evidence for the adequacy of the standard jump-diffusive paradigm for modeling equity index returns remains intact, with the accumulated evidence strongly suggesting that both leverage style effects and jumps are critical ingredients of the return generating mechanism.

\section{Conclusion}

We have introduced a novel three step procedure to explore the general properties of the return generating process underlying a given intraday return series. Each step speaks to the empirical strength of important features of the corresponding daily returns, namely stochastic volatility, the presence of an asymmetric relationship between return and volatility innovations (the leverage effect) and the existence of jumps or extreme outliers in the return distribution. In combination, the procedure may be taken as an informal test for whether the underlying return process belongs to the arbitrage-free class of continuous-time semimartingales. The properties of the relevant test statistics associated with each step of the procedure are explored through an extensive simulation study, where importantly we also consider the impact of microstructure noise. Finally, we show that the empirical behavior of the financial time standardized returns from the S\&P 500 futures equity index market is compatible with a jump-diffusion endowed with a pronounced leverage effect. The associated decomposition into a jump process, an indication of the strength of the leverage effect, and a measure of the integrated variance associated with the diffusive component of the return process should be useful in the formulation of empirically more realistic continuous-time models. For now, we only claim to have shed new light upon the relationship between the recent high-frequency realized volatility based literature and the more traditional daily conditional return distribution literature based on the discrete-time ARCH or stochastic volatility paradigm.

The only preceding studies to focus on similar issues from an empirical perspective are Zhou (1998) and PV (2004), and only the latter pursue formal normality tests. We expand significantly on their work in several important dimensions. First, they provide evidence only for standardized financial time returns over an average of $2^{1 / 2}$ trading days. Given the sample size, our simulations document very poor power properties at this sampling frequency. Second, since they do not study standardized calendar returns 
nor allow for jumps, they fail to elicit evidence regarding the importance of these key distinct features of the return process. Third, they apply only two direct normality tests, namely the KS test and the (adjusted) JB test, supplemented by a tail test and two tests focusing on independence of the standardized financial time return series. Our simulation study finds that none of these tests have any discernable discriminatory power at the relevant sampling frequency. In contrast, we systematically explore the size of a battery of tests for i.i.d. standard normality and study the power of the tests when all relevant features of the return series are accommodated. As a result, we are able to focus our empirical study on a number of wellbehaved test statistics. Consequently, our empirical conclusions also differ in substantial ways. We find that jumps are important and that the null hypothesis of a continuous semi-martingale is strongly rejected. Accounting for the presence of jumps also allows us to draw more firm conclusion about the wider semimartingale class of models consistent with the observed returns. In the course of this analysis, we introduce a novel intraday jump identification and extraction procedure which performs admirably in comparison to the existing BN-S test. We also explore the potential impact of microstructure noise on the inference, suggesting a relatively simple-to-implement strategy for dealing with some of the potential problems encountered in the practical implementation of standard i.i.d. noise adjustment procedures.

A number of issues call out for additional inquiry. First, how widely can these strict tests for normality of the appropriately standardized return series be expected to apply? The S\&P 500 futures series is quite unique in terms of having minimal market microstructure distortions. Second, the various steps involved in our test procedure may be refined or extended in a variety of ways. Third, it is feasible to explore the impact of more complex market microstructure noise and a range of other complications within the simulation setting. Fourth, the decomposition of the return process may be related to other market activity variables within the context of the Mixture-of-Distributions hypothesis, and it would be interesting to study the validity of these predictions more closely. Lastly, further investigation concerning the usefulness of the new distributional results and test statistics for practical financial decision making constitutes another very interesting and important avenue for future research. 


\section{APPENDIX I. Moment-Based Tests}

Let the elements $\tilde{R}_{T, t}$ of the array $\left\{\tilde{R}_{T, t}: T=1,2, \ldots, \infty ; t=1,2, \ldots, T\right\}$ be distributed with pdf $f_{\tilde{R}_{T, t}}^{n_{T, t}}(\tilde{r})=\frac{\Gamma\left(n_{T, t} / 2\right)}{\sqrt{\pi n_{T, t}} \Gamma\left(\left(n_{T, t}-1\right) / 2\right)}\left(1-\frac{\tilde{r}^{2}}{n_{T, t}}\right)^{\frac{\left(n_{T, t}-3\right)}{2}} 1_{\left(-\sqrt{n_{T, t}}, \sqrt{n_{T, t}}\right)}(\tilde{r}), \tilde{r} \in \mathbb{R}, n_{T, t} \in \mathbb{N}$

Lemma 1. Define the raw moments of each array element as $m_{T, t}^{(k)}=\mathrm{E}\left[\tilde{R}_{T, t}^{k}\right]$

Then

$$
m_{T, t}^{(2 k-1)}=0, m_{T, t}^{(2 k)}=\frac{n_{T, t}^{k} \cdot(2 k-1) \cdot(2 k-3) \cdot \ldots \cdot 1}{\left(n_{T, t}+2 k-2\right) \cdot\left(n_{T, t}+2 k-4\right) \cdot \ldots \cdot n_{T, t}}, k=1,2, \ldots
$$

Proof. See PV (2004).

For our purposes each $n_{T, t}$ in physical time equals the sampling frequency, while in financial time it is determined as the random number of consecutive intraday returns whose cumulative quadratic variation attains the desired "day length." Hence, if volatility is assumed, realistically, to be uniformly bounded away from zero a.s. (at least over some part of the trading day), $n_{T, t}$ would necessarily be uniformly bounded a.s. Thus, without loss of generality, the following multivariate CLT holds:

Lemma 2. Define the sample raw moments of each array row as $\quad \bar{M}_{T}^{(k)}=\frac{1}{T} \sum_{t=1}^{T} \tilde{R}_{T, t}^{k} \quad$ with corresponding population values $M_{T}^{(k)}=\mathrm{E}\left[\bar{M}_{T}^{(k)}\right]=\frac{1}{T} \sum_{t=1}^{T} m_{T, t}^{(k)}$. Then $M_{T}^{(1)}=0, M_{T}^{(2)}=1, M_{T}^{(3)}=0$ $M_{T}^{(4)}=\frac{1}{T} \sum_{t=1}^{T} \frac{3 n_{T, t}}{n_{T, t}+2}, M_{T}^{(6)}=\frac{1}{T} \sum_{t=1}^{T} \frac{15 n_{T, t}^{2}}{\left(n_{T, t}+4\right)\left(n_{T, t}+2\right)}, M_{T}^{(8)}=\frac{1}{T} \sum_{t=1}^{T} \frac{105 n_{T, t}^{3}}{\left(n_{T, t}+6\right)\left(n_{T, t}+4\right)\left(n_{T, t}+2\right)}$

Moreover, if $n_{T, t}$ is uniformly bounded a.s. then

$\sqrt{T}\left[\begin{array}{cccc}M_{T}^{(2)} & 0 & M_{T}^{(4)} & 0 \\ 0 & M_{T}^{(4)}-\left[M_{T}^{(2)}\right]^{2} & 0 & M_{T}^{(6)}-M_{T}^{(2)} M_{T}^{(4)} \\ M_{T}^{(4)} & 0 & M_{T}^{(6)} & 0 \\ 0 & M_{T}^{(6)}-M_{T}^{(2)} M_{T}^{(4)} & 0 & M_{T}^{(8)}-\left[M_{T}^{(4)}\right]^{2}\end{array}\right]^{-1 / 2}\left(\begin{array}{l}\bar{M}_{T}^{(1)} \\ \bar{M}_{T}^{(2)}-M_{T}^{(2)} \\ \bar{M}_{T}^{(3)} \\ M_{T}^{(4)}-M_{T}^{(4)}\end{array}\right) \stackrel{d}{\longrightarrow} N\left(0,1_{4 \times 4}\right)$

Proof. Define

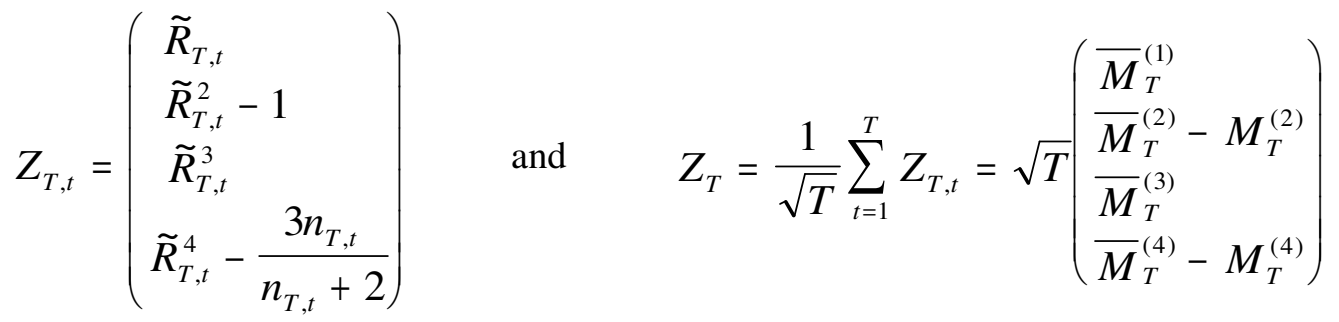


Note that $Z_{T, t}$ is a vector martingale difference and $\mathrm{E}\left[Z_{T} Z_{T}^{\prime}\right]=\Omega_{T}$, where $\Omega_{T}$ is the following positive definite matrix:

$$
\Omega_{T}=\left[\begin{array}{cccc}
M_{T}^{(2)} & 0 & M_{T}^{(4)} & 0 \\
0 & M_{T}^{(4)}-\left[M_{T}^{(2)}\right]^{2} & 0 & M_{T}^{(6)}-M_{T}^{(2)} M_{T}^{(4)} \\
M_{T}^{(4)} & 0 & M_{T}^{(6)} & 0 \\
0 & M_{T}^{(6)}-M_{T}^{(2)} M_{T}^{(4)} & 0 & M_{T}^{(8)}-\left[M_{T}^{(4)}\right]^{2}
\end{array}\right]
$$

Hence, the multivariate CLT that we need to prove can be restated as:

$\Omega_{T}^{-1 / 2} Z_{T} \stackrel{d}{\longrightarrow} N\left(0,1_{4 x 4}\right)$

By the Cramer-Wald device, see, e.g., theorem 25.5 in Davidson (1994), it suffices to demonstrate the following scalar CLT:

$\frac{a^{\prime} \Omega_{T}^{-1 / 2} Z_{T}}{\|a\|} \stackrel{d}{\longrightarrow} N(0,1) \quad \forall a \in R^{4}, a \neq 0$

Define $x_{T, t}^{a}=\frac{a^{\prime} \Omega_{T}^{-1 / 2} Z_{T, t}}{\sqrt{T}\|a\|}$ and note that $x_{T}^{a}=\sum_{t=1}^{T} x_{T, t}^{a}=\sum_{t=1}^{T} \frac{a^{\prime} \Omega_{T}^{-1 / 2} Z_{T, t}}{\sqrt{T}\|a\|}=\frac{a^{\prime} \Omega_{T}^{-1 / 2} Z_{T}}{\|a\|}$

Now (A2) follows by a martingale difference CLT such as 24.3 in Davidson (1994):

$\sum_{t=1}^{T} x_{T, t}^{a} \stackrel{d}{\longrightarrow} N(0,1) \quad \forall a \in R^{4}$

It suffices to confirm that $x_{T, t}^{a}$ is a martingale difference array such that $\sum_{t=1}^{T} \mathrm{E}\left[\left(x_{T, t}^{a}\right)^{2}\right]=1$ and the

following two conditions hold: ( a ) $\sum_{t=1}^{T}\left(x_{T, t}^{a}\right)^{2} \stackrel{P}{\longrightarrow} 1$ and $\quad$ ( b ) $\max _{1 \leq t \leq T}\left|x_{T, t}^{a}\right| \stackrel{P}{\longrightarrow} 0$

Indeed, $x_{T, t}^{a}$ is a martingale difference array because $Z_{T, t}$ is a vector martingale difference. Moreover, this implies also that

$\sum_{t=1}^{T} \mathrm{E}\left[\left(x_{T, t}^{a}\right)^{2}\right]=\mathrm{E}\left[\sum_{t=1}^{T}\left(x_{T, t}^{a}\right)^{2}\right]=\mathrm{E}\left[\left(\sum_{t=1}^{T} x_{T, t}^{a}\right)^{2}\right]=\mathrm{E}\left[\left(\frac{a^{\prime} \Omega_{T}^{-1 / 2} Z_{T}}{\|a\|}\right)\left(\frac{a^{\prime} \Omega_{T}^{-1 / 2} Z_{T}}{\|a\|}\right)^{\prime}\right]=1$

Condition (b) is satisfied since $\sqrt{T} x_{T, t}^{a}$ is uniformly bounded a.s., because the support of $\tilde{R}_{T, t}$ is $\left(-\sqrt{\boldsymbol{n}_{T, t}}, \sqrt{\boldsymbol{n}_{T, t}}\right)$ and $\boldsymbol{n}_{T, t}$ is uniformly bounded a.s. so that both $\Omega_{T}$ and $Z_{T, t}$ are uniformly bounded a.s.

To verify condition (a), observe $T\left[\left(x_{T, t}^{a}\right)^{2}-\mathrm{E}\left[\left(x_{T, t}^{a}\right)^{2}\right]\right]$ is uniformly bounded a.s. Therefore, a martingale LLN (corollary 19.9 in Davidson (1994)) yields condition (a) as follows:

$\sum_{t=1}^{T}\left[\left(x_{T, t}^{a}\right)^{2}-\mathrm{E}\left[\left(x_{T, t}^{a}\right)^{2}\right]\right] \stackrel{P}{\longrightarrow} 0 \Leftrightarrow \sum_{t=1}^{T}\left(x_{T, t}^{a}\right)^{2}-1 \stackrel{P}{\longrightarrow} 0$

Thus, all conditions for the martingale difference CLT are satisfied, implying (A8), (A7) and (A6), QED. 
Corollary 1. The following chi-square test statistics provide different moment-based tests of the null hypothesis that the sample $\tilde{R}_{T, t}, t=1,2, \ldots, T$ is from a distribution with the moments specified above:

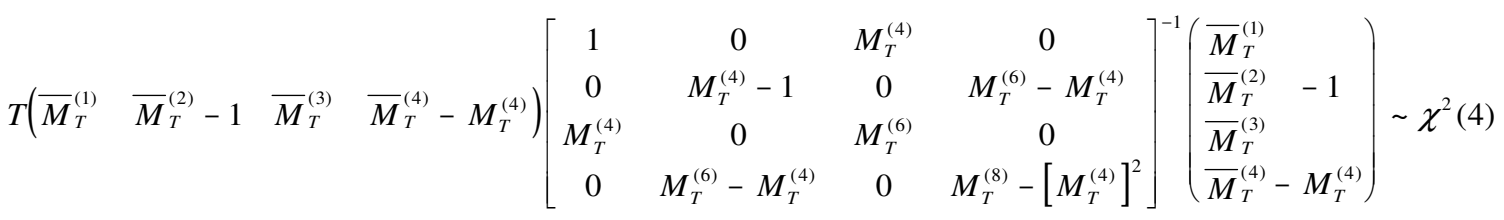

$$
\begin{aligned}
& T \frac{\left[\bar{M}_{T}^{(1)}\right]^{2}}{1} \sim \chi^{2}(1), T \frac{\left[\bar{M}_{T}^{(2)}-1\right]^{2}}{M_{T}^{(4)}-1} \sim \chi^{2}(1), T \frac{\left[\bar{M}_{T}^{(3)}\right]^{2}}{M_{T}^{(6)}} \sim \chi^{2}(1), T \frac{\left[\bar{M}_{T}^{(4)}-M_{T}^{(4)}\right]^{2}}{M_{T}^{(8)}-\left[M_{T}^{(4)}\right]^{2}} \sim \chi^{2}(1) \\
& T\left[\frac{\left[\bar{M}_{T}^{(3)}\right]^{2}}{M_{T}^{(6)}}+\frac{\left[\bar{M}_{T}^{(4)}-M_{T}^{(4)}\right]^{2}}{M_{T}^{(8)}-\left[M_{T}^{(4)}\right]^{2}}\right] \sim \chi^{2}(2)
\end{aligned}
$$

Corollary 2. As $n_{T, t}$ gets large, uniformly for all $t=1,2, \ldots, T$, the moment-based tests become asymptotically equivalent to those for the special limiting case of Gaussian $\tilde{R}_{T, t}$ :

$$
\begin{aligned}
& T\left(\bar{M}_{T}^{(1)} \quad \bar{M}_{T}^{(2)}-1 \quad \bar{M}_{T}^{(3)} \quad \bar{M}_{T}^{(4)}-3\right)\left[\begin{array}{cccc}
1 & 0 & 3 & 0 \\
0 & 2 & 0 & 12 \\
3 & 0 & 15 & 0 \\
0 & 12 & 0 & 96
\end{array}\right]^{-1}\left(\begin{array}{l}
\bar{M}_{T}^{(1)} \\
\bar{M}_{T}^{(2)}-1 \\
\bar{M}_{T}^{(3)} \\
\bar{M}_{T}^{(4)}-3
\end{array}\right) \sim \chi^{2}(4) \\
& T \frac{\left[\bar{M}_{T}^{(1)}\right]^{2}}{1} \sim \chi^{2}(1), T \frac{\left[\bar{M}_{T}^{(2)}-1\right]^{2}}{2} \sim \chi^{2}(1), T \frac{\left[\bar{M}_{T}^{(3)}\right]^{2}}{15} \sim \chi^{2}(1), T \frac{\left[\bar{M}_{T}^{(4)}-M_{T}^{(4)}\right]^{2}}{96} \sim \chi^{2}(1) \\
& T\left[\frac{\left[\bar{M}_{T}^{(3)}\right]^{2}}{15}+\frac{\left[\bar{M}_{T}^{(4)}-M_{T}^{(4)}\right]^{2}}{96}\right] \sim \chi^{2}(2)
\end{aligned}
$$

Corollary 3. The moment-based test for $\mathrm{N}(0,1)$ based on all four moments is identical to the test for $\mathrm{N}(0,1)$ on the corresponding four orthogonal Hermite polynomials spanning the same moments.

Proof. Adapting the BM (2005) Hermitian N(0,1) test to our notation and context it suffices to show that

$$
T\left(\bar{M}_{T}^{(1)} \quad \bar{M}_{T}^{(2)}-1 \quad \bar{M}_{T}^{(3)} \quad \bar{M}_{T}^{(4)}-3\right)\left[\begin{array}{cccc}
1 & 0 & 3 & 0 \\
0 & 2 & 0 & 12 \\
3 & 0 & 15 & 0 \\
0 & 12 & 0 & 96
\end{array}\right]^{-1}\left(\begin{array}{l}
\bar{M}_{T}^{(1)} \\
\bar{M}_{T}^{(2)}-1 \\
\bar{M}_{T}^{(3)} \\
\bar{M}_{T}^{(4)}-3
\end{array}\right)=\sum_{i=1}^{4}\left(\frac{1}{\sqrt{T}} \sum_{t=1}^{T} H_{i}\left(\tilde{R}_{T, t}\right)\right)^{2}
$$

Indeed, the left hand side simplifies to the following quadratic form of moments:

$$
\frac{T}{24}\left(60\left[M_{T}^{(1)}\right]^{2}+48\left[M_{T}^{(2)}\right]^{2}+4\left[M_{T}^{(3)}\right]^{2}+\left[M_{T}^{(4)}\right]^{2}-24\left[M_{T}^{(1)} M_{T}^{(3)}\right]-12\left[M_{T}^{(2)} M_{T}^{(4)}\right]-60\left[M_{T}^{(2)}\right]+6\left[M_{T}^{(4)}\right]+21\right)
$$

The Hermite polynomials on the right hand side produce the expression

$$
T\left(\left[M_{T}^{(1)}\right]^{2}+\frac{1}{2}\left[M_{T}^{(2)}-1\right]^{2}+\frac{1}{6}\left[M_{T}^{(3)}-3 M_{T}^{(1)}\right]^{2}+\frac{1}{24}\left[M_{T}^{(4)}-6 M_{T}^{(2)}+3\right]^{2}\right)
$$

which turns out to be just another representation of the same quadratic form. QED. 


\section{APPENDIX II. Data Description}

\begin{tabular}{llll}
\hline Data Type & Set & \#Samples X \#Days & Description \\
\hline Simulated & $\# 1$ & $1,000 \times 5,000$ & No leverage, no jumps \\
Simulated & $\# 2$ & $1,000 \times 5,000$ & No leverage, large rare jumps \\
Simulated & $\# 3$ & $1,000 \times 5,000$ & No leverage, frequent small jumps \\
Simulated & $\# 4$ & $1,000 \times 5,000$ & No leverage, moderate jumps \\
Simulated & $\# 5$ & $1,000 \times 5,000$ & Leverage, no jumps \\
Simulated & $\# 6$ & $1,000 \times 5,000$ & Leverage, large rare jumps \\
Simulated & $\# 7$ & $1,000 \times 5,000$ & Leverage, frequent small jumps \\
Simulated & \#8 & $1,000 \times 5,000$ & Leverage, moderate jumps \\
Simulated & \#X8 & $1,000 \times 50,000$ & \#8 for ten times larger samples \\
Simulated & \#8n & $1,000 \times 5,000$ & \#8 under Gaussian i.i.d. microstructure noise \\
Simulated & \#X8n & $1,000 \times 50,000$ & \#X8 under Gaussian i.i.d. microstructure noise \\
Real Data & SP500 & $1 \times 4,126$ & S\&P 500 most liquid futures from $1 / 1 / 88$ to $7 / 26 / 04$ \\
\hline
\end{tabular}

\section{Simulated data.}

We generate samples from eight specifications of a one-factor jump-diffusion model with Poisson jumps of constant intensity $\lambda$ and lognormal jump size $\kappa$ with variance $\hat{v}^{2}$ :

$d p(t)=\sigma(t) d W_{1}(t)+\kappa d q_{t}$,

$d \sigma^{2}(t)=\eta\left(\theta-\sigma^{2}(t)\right) d t+v \sigma(t) d W_{2}(t)$

The diffusive volatility parameters are set to $\theta=1, \eta=0.01, v=0.1$. We simulate four jump scenarios first without leverage and then with leverage by setting $\operatorname{corr}\left(d W_{1}(t), d W_{2}(t)\right)=-0.5$ :

\begin{tabular}{llc}
\hline Jump scenario & Jump parameters & $\begin{array}{c}\text { Jump contribution } \\
\text { to total volatility }\end{array}$ \\
\hline No jumps & $\widehat{\mathrm{v}}^{2}=0, \lambda=0$ & $0 \%$ \\
Large rare jumps & $\widehat{\mathrm{v}}^{2}=2.5, \lambda=0.1$ & $20 \%$ \\
Small frequent jumps & $\widehat{\mathrm{v}}^{2}=0.25, \lambda=1.0$ & $20 \%$ \\
Moderate jumps & $\widehat{\mathrm{v}}^{2}=0.5, \lambda=0.2$ & $9.1 \%$ \\
\hline
\end{tabular}

The simulations are based on an Euler scheme, the simulation frequency is 1 second, while the sampling frequency is 120 seconds with a total of 195 sample returns per day. For scenarios \#1 - \#8 we simulate $5,000,000$ days providing 1,000 high-frequency data samples with 5,000 days each. Scenario \#X8 is the same as \#8 but each of the 1,000 samples has ten times larger size 50,000 days, i.e. 50,000,000 days in total. Finally, we simulate scenarios \#8n and \#X8n that replicate \#8 and \#X8 under Gaussian i.i.d. microstructure noise at the sampling frequency with noise-to-signal ratio set to 0.0001 (noise variance realtive to the daily diffusive component of daily return variation). 


\section{Real data.}

We use the R \& C Research tick transaction data for the S\&P 500 stock index futures contract traded on the Chicago Merchantile Exchange. The sample spans January 1, 1988 to July 26, 2004. We construct two minute returns by the previous tick method from the recorded transaction prices between 9:30 and 16:00 EST for the most liquid futures contract (with shortest maturity above five busines days). We exclude days beginning or ending with ten or more zero two-minute returns. After excluding 44 such days we are left with 4,126 trading days.

\section{Sampling procedure in feasible financial time}

Given a sample of intraday returns, we measure feasible financial time in units of the average daily realized volatility for the sample. Then we sample in periods of length $0.5,1$, and 2.5 units of financial time by the following procedure:

1. Set the financial time clock to zero;

2. Increment the financial time clock by the square of the next intraday return in the sample (and subtract twice the noise variance to correct for microstructure effects, if necessary);

3. Repeat step 2 until the clock reaches/exceeds the chosen period length;

4. Sample, increment the period count, and return to step 1;

The sample points obtained following this procedure define "days" in financial time. However, unlike in physical time, the days in financial time have a different numbers of intraday returns and almost the same volatility level, unless significant diffusive return outliers or undetected jumps induce spikes in the measurement of financial time (see tables V.1. and V.2.)

\section{Adjusting the jump extraction procedure for intraday volatility pattern}

First, the intraday volatility pattern is approximated by the average squared return at each of the 195 intraday sample points across the ful sample. Each sqaured intraday return is simply divided by the corresponding average squared return for that interval. Then jump-detection is performed on the patternadjusted return series to find statistically significant jumps that are not driven by the identified pattern. The actual jump size is restored by scaling back the adjusted series or, equivalently, by scanning the original series at locations identified as jumps in the pattern-adjusted series. 


\section{Acknowledgments}

This research was supported by a grant from the National Science Foundation to the NBER for Andersen and Bollerslev. We would like to thank two anonymous referees, Christian Bontemps, and Nour Meddahi for many helpful suggestions, which greatly improved the papers. We also thank participants at the International Finance Conference at the University of Copenhagen, September 2005, and seminar participants at the Robert H. Smith School, University of Maryland and the University of Wisconsin at Madison for comments. 


\section{References}

Aït-Sahalia, Y., 2002, Telling from Discrete Data Whether the Underlying Continuous-Time Model is a Diffusion. Journal of Finance 57, 2075-2121.

Aït-Sahalia, Y., P.A. Mykland and L. Zhang, 2005, How Often to Sample a Continuous-Time Process in the Presence of Market Microstructure Noise. Review of Financial Studies 18, 351-416.

Alizadeh, S., M. Brandt and F.X. Diebold, 2002, Range-Based Estimation of Stochastic Volatility Models. Journal of Finance 57, 1047-1091.

Andersen, T.G., 1992, Volatility. Manuscript, Northwestern University.

Andersen, T.G., 1996, Return Volatility and Trading Volume: An Information Flow Interpretation of Stochastic Volatility. Journal of Finance 51, 169-204.

Andersen, T.G., L. Benzoni and J. Lund, 2002, Estimating Jump-Diffusions for Equity Returns. Journal of Finance 57, 1239-1284.

Andersen, T.G. and T. Bollerslev, 1997a, Heterogeneous Information Arrivals and Return Volatility Dynamics: Uncovering the Long-Run in High-Frequency Returns. Journal of Finance 52, 975-1005.

Andersen, T.G. and T. Bollerslev, 1997b, Intraday Periodicity and Volatility Persistence in Financial Markets. Journal of Empirical Finance 4, 115-158.

Andersen, T.G. and T. Bollerslev, 1998, Answering the Skeptics: Yes, Standard Volatility Models Do Provide Accurate Forecasts. International Economic Review 39, 885-905.

Andersen, T.G., T. Bollerslev and F.X. Diebold, 2004, Parametric and Non-Parametric Volatility Measurement, in: L.P Hansen and Y. Aït-Sahalia, (Eds.), Handbook of Financial Econometrics, Elsevier Science, New York, forthcoming.

Andersen, T.G., T. Bollerslev, F.X. Diebold and H. Ebens, 2001, The Distribution of Realized Stock Return Volatility. Journal of Financial Economics 61, 43-76.

Andersen, T.G., T. Bollerslev, F.X. Diebold and P. Labys, 2000, Great Realizations. Risk 13, 105-108.

Andersen, T.G., T. Bollerslev, F.X. Diebold and P. Labys, 2001, The Distribution of Realized Exchange Rate Volatility. Journal of the American Statistical Association 96, 42-55.

Andersen, T.G., T. Bollerslev, F.X. Diebold and P. Labys, 2003, Modeling and Forecasting Realized Volatility. Econometrica 71, 579-625.

Andersen, T.G., T. Bollerslev and F.X. Diebold, 2005, Roughing It Up: Including Jump Components in the Measurement, Modeling and Forecasting of Return Volatility. Manuscript, Northwestern University, Duke University, and University of Pennsylvania.

Andersen, T.G., T. Bollerslev, F.X. Diebold and C. Vega, 2003, Micro Effects of Macro Announcements: Real-Time Price Discovery in Foreign Exchange. American Economic Review 93, 38-62.

Andersen, T.G., T. Bollerslev, F.X. Diebold and C. Vega, 2005, Real-Time Price Discovery in Stock, Bond and Foreign Exchange Markets. Manuscript, Northwestern University, Duke University, University of Pennsylvania, and University of Rochester. 
Andersen, T.G., T. Bollerslev, P.H. Frederiksen and M.Ø. Nielsen, 2005, Jumps in Common Stock Returns. Manuscript, Northwestern University, Duke University, and Cornell University.

Andersen, T.G., T. Bollerslev, P.H. Frederiksen and M.Ø. Nielsen, 2006, Comment on Realized Variance and Market Microstructure Noise, by P.R. Hansen and A. Lunde. Journal of Business and Economic Statistics, forthcoming.

Andersen, T.G., T. Bollerslev and N. Meddahi, 2004, Analytic Evaluation of Volatility Forecasts. International Economic Review 45, 1079-1110.

Andersen, T.G., T. Bollerslev and N. Meddahi, 2005, Correcting the Errors: Volatility Forecast Evaluation using High-frequency Data and Realized Volatilities. Econometrica 72, 279-296.

Ané, T. And H. Geman, 2000, Order Flow, Transaction Clock, and Normality of Asset Returns. Journal of Finance $55,2259-2284$.

Back, K., 1991, Asset Prices for General Processes. Journal of Mathematical Economics 20, 317-395.

Ball, C.A. and W.N. Torous, 1983, A Simplified Jump Process for Common Stock Returns. Journal of Financial and Quantitative Analysis 18, 53-65.

Bandi, F.M. and J.R. Russell, 2004a, Microstructure Noise, Realized Volatility, and Optimal Sampling. Manuscript, University of Chicago.

Bandi, F.M. and J.R. Russell, 2004b, Separating Microstructure Noise from Volatility. Manuscript, University of Chicago.

Barndorff-Nielsen, O.E. and N. Shephard, 2001, Non-Gaussian Ornstein-Uhlenbeck-based Models and Some of Their Uses in Financial Economics. Journal of the Royal Statistical Society Series B 63, 167-241.

Barndorff-Nielsen, O.E. and N. Shephard, 2002a, Econometric Analysis of Realised Volatility and its use in Estimating Stochastic Volatility Models. Journal of the Royal Statistical Society 64, 253-280.

Barndorff-Nielsen, O.E. and N. Shephard, 2002b, Estimating Quadratic Variation Using Realized Variance. Journal of Applied Econometrics 17, 457-478.

Barndorff-Nielsen, O.E. and N. Shephard, 2004, Power and Bipower Variation with Stochastic Volatility and Jumps. Journal of Financial Econometrics 2, 1-37.

Barndorff-Nielsen, O.E. and N. Shephard, 2005, Econometrics of Testing for Jumps in Financial Economics Using Bipower Variation. Journal of Financial Econometrics, forthcoming.

Bates, D.S., 2000, Post- 87 Crash fears in the S\&P500 Futures Option Market. Journal of Econometrics 94, 181-238.

Bekaert, G. and G. Wu, 2000, Asymmetric Volatility and Risk in Equity Markets. Review of Financial Studies 13, 142.

Bollen, B. and B. Inder, 2002, Estimating Daily Volatility in Financial Markets Utilizing Intraday Data. Journal of Empirical Finance 9, 551-562.

Bollerslev, T., 1987, A Conditionally Heteroskedastic Time Series Model for Speculative Prices and Rates of Return. Review of Economics and Statistics 69, 542-547.

Bollerslev, T., J. Litvinova and G. Tauchen, 2005, Leverage and Volatility Feedback Effects in High-Frequency Data. 
Manuscript, Duke University.

Bontemps, C. and N. Meddahi, 2002, Testing Normality: A GMM Approach. Manuscript, CIRANO, Montrèal.

Bontemps, C. and N. Meddahi, 2005, Testing Normality: A GMM Approach. Journal of Econometrics 124, 149-186.

Campbell, J.Y. and L. Hentschel, 1992, No News is Good News: An Asymmetric Model of Chnaging Volatility in Stock Returns. Journal of Financial Economics 31, 282-331.

Carr, P., H. Geman, D. Madan and M. Yor, 2002, The Fine Structure of Asset Returns: An Empirical Investigation. Journal of Business 75, 305-332.

Carr, P., H. Geman, D. Madan and M. Yor, 2003, Stochastic Volatility for Lévy Processes. Mathematical Finance 13, 345-382.

Chan, W.H. and J.M. Maheu, 2002, Conditional Jump Dynamics in Stock Market Returns. Journal of Business and Economic Statistics 20, 377-389.

Chang, Y. and J.Y. Park, 2004, Taking a New Contour: A Novel View on Unit Root Tests. Manuscript, Rice University.

Chernov, M., A.R. Gallant, E. Ghysels, and G. Tauchen, 2003, Alternative Models for Stock Price Dynamics. Journal of Econometrics 116, 225-257.

Chib, S., F. Nardari and N. Shephard, 2002, Markov Chain Monte Carlo Methods for Stochastic Vlatility Models. Journal of Econometrics 108, 281-316.

Clark, P.K., 1973, A Subordinate Stochastic Process Model with Finite Variance for Speculative Prices. Econometrica 41, 135-155.

Comte, F. and E. Renault, 1998, Long Memory in Continuous Time Stochastic Volatility Models. Mathematical Finance 8, 291-323.

Corsi, F., Zumbach, U.A. Müller and M. Dacorogna, 2001, Consistent High-Precision Volatility from High-frequency Data. Economic Notes 30, 183-204.

Dacorogna, M.M., R. Gencay, U.A. Müller, O.V. Pictet and R.B. Olsen, 2001. An Introduction to High-Frequency Finance. San Diego: Academic Press.

Dambis, K.E., 1965, On the Decomposition of Continuous Submartingales. Theory of Probability Applications 10, 401-410.

Davidson, J., 1994. Stochastic Limit Theory. New York: Oxford University Press.

Drost, F.C., T.E. Nijman and B.J.M. Werker, 1998, Estimation and Testing in Models Containing Both Jumps and Conditional Heteroskedasticity. Journal of Business and Economic Statistics 16, 237-243.

Dubins, L.E. and G. Schwartz, 1965, On Continuous Martingales. Proceedings of the National Academy of Sciences 53, 913-916.

Epps, T.W. and M.L. Epps, 1976, The Stochastic Dependence of Security Price Changes and Transaction Volumes: Implications for the Mixture-of-Distributions Hypothesis. Econometrica 44, 305-321. 
Eraker, B., 2004, Do Stock Prices and Volatility Jump? Reconciling Evidence from Spot and Option Prices. Journal of Finance 59, 1367-1403.

Eraker, B., M.S. Johannes and N.G. Polson, 2003, The Impact of Jumps in Volatility. Journal of Finance 58, 12691300 .

Forsberg, L. and T. Bollerslev, 2002, Bridging the Gap Between the Distribution of Realized (ECU) Volatility and ARCH Modeling (of the Euro): The GARCH-NIG Model. Journal of Applied Econometrics 17, 535-548.

Gallant, A.R., C.T. Hsu and G. Tauchen, 1999, Using Daily Range Data to Calibrate Volatility Diffusions and Extract the Forward Integrated Variance. Review of Economics and Statistics 81, 617-631.

Garman, M.B. and M.J. Klass, 1980, On the Estimation of Price Volatility from Historical Data. Journal of Business $53,67-78$.

Gillemot, L., J.D. Farmer and F. Lillo, 2005, There's More to Volatility Than Volume. Manuscript, Santa Fe Institute.

Gençay, R. and F. Selçuk, 2004, Volatility-Return Dynamics Across Different Timescales. Manuscript, Simon Fraser University.

Hansen, P.R. and A. Lunde, 2006, Realized Variance and Market Microstructure Noise. Journal of Business and Economic Statistics, forthcoming.

Harris, L., 1986, A Transaction Data Study of Weekly and Intradaily Patterns in Stock Returns. Journal of Financial Economics 16, 99-117.

Huang, X. and G. Tauchen, 2005, The Relative Contribution of Jumps to Total Price Variance. Journal of Financial Econometrics 3, 456-499.

Jarque, C. and A. Bera, 1980, Efficient Tests for Normality, Heteroskedasticity, and Serial Independence or Regression residuals. Economic Letters 6, 255-259.

Johannes, M., 2004, The Statistical and Economic Role of Jumps in Continuous-Time Interest Rate Models. Journal of Finance 59, 227-260.

Karatzas, I. And S.E. Shreve, 1991. Brownian Motion and Stochastic Calculus, $2{ }^{\text {nd }}$ ed. Springer Verlag, New York.

Lai, T.L. and D. Siegmund, 1983, Fixed Accuracy Estimation of an Autoregressive Parameter. Annals of Statistics 11, 478-485.

Maheu, J.M. and T.H. McCurdy, 2004, News Arrival, Jump Dynamics and Volatility Components for Individual Stock Returns. Journal of Finance 59, 755-793.

Mancini, C., 2004, Estimation of the Parameters of Jump of a General Poisson-Diffusion Model. Scandinavian Actuarial Journal 104, 42-52.

Mancini, C., 2005a, Disentangling the Jumps of the Diffusion in a geometric Jumping Brownian Motion. Manuscript, Universita di Firenze.

Mancini, C., 2005b, Estimating the Integrated Volatility in Stochastic Volatility Models with Levy Typw Jumps. Manuscript, Universita di Firenze.

Meddahi, N., 2002, A Theoretical Comparison Between Integrated and Realized Volatility. Journal of Applied Econometrics 17, 479-508. 
Merton, R.C., 1976, Option Pricing when Underlying Stock Returns are Discontinuous. Journal of Financial Economics 3, 125-144.

Nelson, D.B., 1990, ARCH Models as Diffusion Approximations. Journal of Econometrics 45, 7-38.

Nelson, D.B., 1991, Conditional Heteroskedasticity in Asset Returns: A New Approach. Econometrica 59, 347-370.

Nelson, D.B., 1992, Filtering and Forecasting with Misspecified ARCH Models I: Getting the Right Variance with the Wrong Model. Journal of Econometrics 52, 61-90.

Oomen, R.C.A., 2005, Properties of Biased-Corrected Realized Variance Under Alternative Sampling Schemes. Journal of Financial Econometrics 4, 555-577.

Oomen, R.C.A., 2006, Properties of Realized Variance Under Alternative Sampling Schemes. Journal of Business \& Economic Statistics, forthcoming.

Pan, J., 2002, The Jump-Risk Premia Implicit in Options: Evidence from an Integrated Time Series Study. Journal of Financial Economics 63, 3-50.

Parkinson, M., 1980, The Extreme Value Method for Estimating the Variance of the Rate of Return. Journal of Business 53, 61-65.

Peters, R.T. and R.G. de Vilder, 2004, Testing the Diffusion Model for the S\&P500. Manuscript, University of Amsterdam.

Tauchen, G. and M. Pitts, 1983, The Price Variability-Volume Relationship on Speculative Markets. Econometrica $51,485-505$.

Todorov, V. and G. Tauchen, 2005, Simulation Methods for Lévy-Driven CARMA Stochastic Volatility Models. Manuscript, Duke University.

Wood, R.A., T.H. McInish and J.K. Ord, 1985, An Investigation of Transaction Data for NYSE Stocks. Journal of Finance 25, 723-739.

Zhang, L., Y. Ait-Sahalia and P.A. Mykland, 2005, A Tale of Two Time Scales: Determining Integrated Volatility with Noisy High-Frequency Data. Journal of the American Statistical Association, forthcoming.

Zhou, B., 1996, High-Frequency Data and Volatility in Foreign Exchange Rates. Journal of Business and Economic Statistics 14, 45-52.

Zhou, B., 1998, F-Consistency, De-Volatilization and Normalization of High-Frequency Financial Data, in: C. Dunis and B. Zhou, (Eds.), Nonlinear Modelling of High Frequency Financial Time Series, John Wiley Sons Ltd., London. 


\section{Table Notes}

\section{Table I}

"Set" is the simulation set \# described in Appendix II;

"Jump Intensity" and "Jump Variance" are jump parameters of the corresponding simulation set;

"True JV" is the simulated average daily jump volatility;

"Det JV" is the detected average daily jump volatility;

"RMSE" is the square root of the mean square difference between simulated and detected JV for all sample days;

"Eff Daily Size \%" is calculated as the percentage ratio of the number of days, having detected JV $>0$ and true JV=0, to the number of days, having true $\mathrm{JV}=0$;

"Eff Daily Power \%" is calculated as the percentage ratio of the number of days, having detected JV $>0$ and true JV $>0$, to the number of days, having true $\mathrm{JV}>0$.

Tables II.1.A.-II.1.B.

"Set" is the simulation set \# described in Appendix II;

"Days" is the daily sample length as a fraction of one trading day in physical/financial time;

"Mean", "Std", "Skew", "Kurt" are the sample mean, standard deviation, skewness, and kurtosis;

"Kurt*" is the adjusted kurtosis implied by the finite sample distribution (Lemma 1 in Appendix I).

Tables II.2.A.-II.2.B.

"Set" is the simulation set \# described in Appendix II;

"Days" is the daily sample length as a fraction of one trading day in physical/financial time;

"KS" is the rejection frequency of the Kolmogorov-Smirnov test for $\mathrm{N}(0,1)$;

"CVM" is the rejection frequency of the Cramer-Von Mises test for $\mathrm{N}(0,1)$;

"AD" is the rejection frequency of the Anderson-Darling test for $\mathrm{N}(0,1)$;

"JB" is the rejection frequency of the Jarque-Bera test for normality;

"JB Adj" is the rejection frequency of the Jarque-Bera test with informal kurtosis adjustment implied by the finite sample distribution (Lemma 1 in Appendix I).

Tables II.3.A.-II.3.B.

"Set" is the simulation set \# described in Appendix II;

"Days" is the daily sample length as a fraction of one trading day in physical/financial time;

"1", " 2 ", " 3 ", " 4 ", " $3-4 "$, " $1-4$ " are the rejection frequencies of the corresponding moment-based tests for N $(0,1)$ described

in Corollary 2, Appendix I.

Tables II.4.A.-II.4.B.

"Set" is the simulation set \# described in Appendix II;

"Days" is the daily sample length as a fraction of one trading day in physical/financial time;

"1", "2", "3", "4", "3-4", "1-4" are the rejection frequencies of the corresponding adjusted moment-based tests for the finite sample distribution described in Corollary 1, Appendix I.

Tables III.1.-III.3.

"Set" is the simulation set \# described in Appendix II;

"Days" is the daily sample length as a fraction of one trading day in physical/financial time;

The columns in each of the four subsections are the same as the corresponding columns in Tables II.1-II.4.

Tables IV.1.-IV.3.

"Set" is the S\&P 500 real dataset described in Appendix II;

"Days" is the daily sample length as a fraction of one trading day in physical/financial time;

The columns in each of the four subsections are the same as the corresponding columns in Tables II. and III. and the reported numbers for each test are the obtained p-values.

Tables V.1.-V.2.

"Day Length in Phys. Time" gives the mean count of 2-min returns across all days of the sampling scheme;

"Day Length in Fin. Time" gives the mean (std. dev.) of the realized variance across all days of the scheme. 
Table I. Jump Detection Efficiency on Simulated Data: Standard Daily vs. New Intraday Procedure

Standard Daily Jump Detection Procedure: Effective Size, Power and RMSE for Daily Test Size 0.1\%

\begin{tabular}{cccccccc}
\hline Set & Jump Intensity & Jump Variance & True JV & Det JV & RMSE & Eff Daily Size \% & Eff Daily Power \% \\
\hline$\# 1$ & 0.00 & 0.00 & 0.000 & 0.000 & 0.009 & 0.160 & - \\
$\# 2$ & 0.10 & 2.50 & 0.250 & 0.228 & 0.133 & 0.160 & 74.446 \\
$\# 3$ & 1.00 & 0.25 & 0.250 & 0.170 & 0.174 & 0.157 & 44.015 \\
$\# 4$ & 0.20 & 0.50 & 0.100 & 0.078 & 0.096 & 0.159 & 49.501 \\
$\# 5$ & 0.00 & 0.00 & 0.000 & 0.000 & 0.009 & 0.158 & - \\
$\# 6$ & 0.10 & 2.50 & 0.250 & 0.228 & 0.133 & 0.160 & 74.472 \\
$\# 7$ & 1.00 & 0.25 & 0.250 & 0.170 & 0.174 & 0.157 & 44.028 \\
$\# 8$ & 0.20 & 0.50 & 0.100 & 0.078 & 0.096 & 0.164 & 49.496 \\
\hline
\end{tabular}

New Intraday Jump Detection Procedure: Effective Size, Power and RMSE for Daily Test Size 0.1\%

\begin{tabular}{cccccccc}
\hline Set & Jump Intensity & Jump Variance & True JV & Det JV & RMSE & Eff Daily Size \% & Eff Daily Power \% \\
\hline$\# 1$ & 0.00 & 0.00 & 0.000 & 0.000 & 0.005 & 0.153 & - \\
$\# 2$ & 0.10 & 2.50 & 0.250 & 0.250 & 0.078 & 0.149 & 84.869 \\
$\# 3$ & 1.00 & 0.25 & 0.250 & 0.236 & 0.093 & 0.153 & 65.397 \\
$\# 4$ & 0.20 & 0.50 & 0.100 & 0.098 & 0.051 & 0.149 & 68.428 \\
$\# 5$ & 0.00 & 0.00 & 0.000 & 0.000 & 0.005 & 0.153 & - \\
$\# 6$ & 0.10 & 2.50 & 0.250 & 0.250 & 0.078 & 0.151 & 84.893 \\
$\# 7$ & 1.00 & 0.25 & 0.250 & 0.236 & 0.092 & 0.148 & 65.405 \\
$\# 8$ & 0.20 & 0.50 & 0.100 & 0.098 & 0.051 & 0.152 & 68.437 \\
\hline
\end{tabular}

New Intraday Jump Detection Procedure: Effective Size, Power and RMSE for Daily Test Size 0.001\%

\begin{tabular}{cccccccc}
\hline Set & Jump Intensity & Jump Variance & True JV & Det JV & RMSE & Eff Daily Size \% Eff Daily Power \% \\
\hline$\# 1$ & 0.00 & 0.00 & 0.000 & 0.000 & 0.001 & 0.002 & - \\
$\# 2$ & 0.10 & 2.50 & 0.250 & 0.249 & 0.079 & 0.002 & 81.912 \\
$\# 3$ & 1.00 & 0.25 & 0.250 & 0.224 & 0.109 & 0.003 & 57.994 \\
$\# 4$ & 0.20 & 0.50 & 0.100 & 0.096 & 0.055 & 0.003 & 62.468 \\
$\# 5$ & 0.00 & 0.00 & 0.000 & 0.000 & 0.001 & 0.003 & - \\
$\# 6$ & 0.10 & 2.50 & 0.250 & 0.249 & 0.079 & 0.002 & 81.915 \\
$\# 7$ & 1.00 & 0.25 & 0.250 & 0.224 & 0.109 & 0.002 & 58.000 \\
$\# 8$ & 0.20 & 0.50 & 0.100 & 0.096 & 0.055 & 0.003 & 62.476 \\
\hline
\end{tabular}

The standard daily jump detection procedure is based on the ratio jump statistics with maximum adjustment as in Barndorff-Nielsen and Shephard (2006). The new intraday procedure is described in the jump detection section of the paper. 
Table II.1A. Descriptive Statistics for Simulated Datasets Without Leverage

\begin{tabular}{|c|c|c|c|c|c|c|c|c|c|c|c|}
\hline Set & Days & Mean & StdD & Skew & Kurt & Kurt $^{*}$ & Mean & StdD & Skew & Kurt & Kurt $^{*}$ \\
\hline \multirow[t]{8}{*}{ \#1 } & & \multicolumn{5}{|c|}{ Physical Time Before Jump Detection } & \multicolumn{5}{|c|}{ Financial Time Before Jump Detection } \\
\hline & 0.5 & 0.00 & 1.00 & -0.00 & 2.94 & 2.94 & 0.00 & 1.00 & -0.00 & 2.91 & 2.91 \\
\hline & 1.0 & 0.00 & 1.00 & 0.00 & 2.97 & 2.97 & 0.00 & 1.00 & -0.00 & 2.95 & 2.96 \\
\hline & 2.5 & 0.00 & 1.00 & 0.00 & 2.98 & 2.99 & 0.00 & 1.00 & -0.00 & 2.98 & 2.98 \\
\hline & & \multicolumn{5}{|c|}{ Physical Time After Jump Detection } & \multicolumn{5}{|c|}{ Financial Time After Jump Detection } \\
\hline & 0.5 & 0.00 & 1.00 & -0.00 & 2.94 & 2.94 & 0.00 & 1.00 & -0.00 & 2.91 & 2.91 \\
\hline & 1.0 & 0.00 & 1.00 & 0.00 & 2.97 & 2.97 & 0.00 & 1.00 & -0.00 & 2.95 & 2.96 \\
\hline & 2.5 & 0.00 & 1.00 & 0.00 & 2.98 & 2.99 & 0.00 & 1.00 & -0.00 & 2.98 & 2.98 \\
\hline \multirow[t]{8}{*}{ \#2 } & & \multicolumn{5}{|c|}{ Physical Time Before Jump Detection } & \multicolumn{5}{|c|}{ Financial Time Before Jump Detection } \\
\hline & 0.5 & -0.00 & 1.00 & -0.00 & 2.89 & 2.94 & -0.00 & 1.00 & -0.00 & 2.86 & 2.92 \\
\hline & 1.0 & -0.00 & 1.00 & 0.00 & 2.90 & 2.97 & -0.00 & 1.00 & -0.00 & 2.86 & 2.96 \\
\hline & 2.5 & -0.00 & 1.00 & 0.00 & 2.88 & 2.99 & -0.00 & 1.00 & 0.00 & 2.83 & 2.98 \\
\hline & & \multicolumn{5}{|c|}{ Physical Time After Jump Detection } & \multicolumn{5}{|c|}{ Financial Time After Jump Detection } \\
\hline & 0.5 & -0.00 & 1.00 & -0.00 & 2.94 & 2.94 & -0.00 & 1.00 & -0.00 & 2.91 & 2.91 \\
\hline & 1.0 & -0.00 & 1.00 & 0.00 & 2.97 & 2.97 & -0.00 & 1.00 & -0.00 & 2.95 & 2.96 \\
\hline & 2.5 & -0.00 & 1.00 & 0.00 & 2.98 & 2.99 & -0.00 & 1.00 & 0.00 & 2.98 & 2.98 \\
\hline \multirow[t]{8}{*}{ \#3 } & & \multicolumn{5}{|c|}{ Physical Time Before Jump Detection } & \multicolumn{5}{|c|}{ Financial Time Before Jump Detection } \\
\hline & 0.5 & 0.00 & 1.00 & -0.00 & 2.82 & 2.94 & -0.00 & 1.00 & -0.00 & 2.77 & 2.91 \\
\hline & 1.0 & 0.00 & 1.00 & 0.00 & 2.84 & 2.97 & 0.00 & 1.00 & 0.00 & 2.82 & 2.96 \\
\hline & 2.5 & 0.00 & 1.00 & 0.00 & 2.88 & 2.99 & 0.00 & 1.00 & 0.00 & 2.90 & 2.98 \\
\hline & & \multicolumn{5}{|c|}{ Physical Time After Jump Detection } & \multicolumn{5}{|c|}{ Financial Time After Jump Detection } \\
\hline & 0.5 & 0.00 & 1.00 & -0.00 & 2.93 & 2.94 & -0.00 & 1.00 & 0.00 & 2.90 & 2.91 \\
\hline & 1.0 & 0.00 & 1.00 & 0.00 & 2.97 & 2.97 & -0.00 & 1.00 & 0.00 & 2.95 & 2.96 \\
\hline & 2.5 & 0.00 & 1.00 & 0.00 & 2.98 & 2.99 & -0.00 & 1.00 & 0.00 & 2.97 & 2.98 \\
\hline \multirow[t]{8}{*}{ \#4 } & & \multicolumn{5}{|c|}{ Physical Time Before Jump Detection } & \multicolumn{5}{|c|}{ Financial Time Before Jump Detection } \\
\hline & 0.5 & 0.00 & 1.00 & -0.00 & 2.89 & 2.94 & 0.00 & 1.00 & -0.00 & 2.86 & 2.92 \\
\hline & 1.0 & 0.00 & 1.00 & -0.00 & 2.91 & 2.97 & 0.00 & 1.00 & -0.00 & 2.89 & 2.96 \\
\hline & 2.5 & 0.00 & 1.00 & 0.00 & 2.93 & 2.99 & 0.00 & 1.00 & -0.00 & 2.92 & 2.98 \\
\hline & & \multicolumn{5}{|c|}{ Physical Time After Jump Detection } & \multicolumn{5}{|c|}{ Financial Time After Jump Detection } \\
\hline & 0.5 & 0.00 & 1.00 & -0.00 & 2.94 & 2.94 & 0.00 & 1.00 & 0.00 & 2.91 & 2.91 \\
\hline & 1.0 & 0.00 & 1.00 & -0.00 & 2.97 & 2.97 & 0.00 & 1.00 & 0.00 & 2.95 & 2.96 \\
\hline & 2.5 & 0.00 & 1.00 & 0.00 & 2.99 & 2.99 & 0.00 & 1.00 & -0.00 & 2.98 & 2.98 \\
\hline
\end{tabular}


Table II.1B. Descriptive Statistics for Simulated Datasets With Leverage

\begin{tabular}{|c|c|c|c|c|c|c|c|c|c|c|c|}
\hline Set & Days & Mean & StdD & Skew & Kurt & Kurt $^{\star}$ & Mean & StdD & Skew & Kurt & Kurt $^{*}$ \\
\hline \multirow[t]{8}{*}{ \#5 } & & \multicolumn{5}{|c|}{ Physical Time Before Jump Detection } & \multicolumn{5}{|c|}{ Financial Time Before Jump Detection } \\
\hline & 0.5 & 0.01 & 1.00 & -0.00 & 2.94 & 2.94 & 0.00 & 1.00 & 0.00 & 2.91 & 2.91 \\
\hline & 1.0 & 0.02 & 1.00 & 0.00 & 2.97 & 2.97 & 0.00 & 1.00 & 0.00 & 2.95 & 2.96 \\
\hline & 2.5 & 0.02 & 1.00 & -0.00 & 2.99 & 2.99 & 0.00 & 1.00 & -0.00 & 2.97 & 2.98 \\
\hline & & \multicolumn{5}{|c|}{ Physical Time After Jump Detection } & \multicolumn{5}{|c|}{ Financial Time After Jump Detection } \\
\hline & 0.5 & 0.01 & 1.00 & -0.00 & 2.94 & 2.94 & 0.00 & 1.00 & 0.00 & 2.91 & 2.91 \\
\hline & 1.0 & 0.02 & 1.00 & 0.00 & 2.97 & 2.97 & 0.00 & 1.00 & 0.00 & 2.95 & 2.96 \\
\hline & 2.5 & 0.02 & 1.00 & -0.00 & 2.99 & 2.99 & 0.00 & 1.00 & -0.00 & 2.97 & 2.98 \\
\hline \multirow[t]{8}{*}{ \#6 } & & \multicolumn{5}{|c|}{ Physical Time Before Jump Detection } & \multicolumn{5}{|c|}{ Financial Time Before Jump Detection } \\
\hline & 0.5 & 0.01 & 1.00 & -0.00 & 2.90 & 2.94 & -0.00 & 1.00 & 0.00 & 2.86 & 2.92 \\
\hline & 1.0 & 0.01 & 1.00 & 0.00 & 2.90 & 2.97 & -0.00 & 1.00 & 0.00 & 2.87 & 2.96 \\
\hline & 2.5 & 0.02 & 1.00 & 0.00 & 2.88 & 2.99 & -0.00 & 1.00 & -0.00 & 2.84 & 2.98 \\
\hline & & \multicolumn{5}{|c|}{ Physical Time After Jump Detection } & \multicolumn{5}{|c|}{ Financial Time After Jump Detection } \\
\hline & 0.5 & 0.01 & 1.00 & -0.00 & 2.94 & 2.94 & 0.00 & 1.00 & 0.00 & 2.91 & 2.91 \\
\hline & 1.0 & 0.01 & 1.00 & 0.00 & 2.97 & 2.97 & 0.00 & 1.00 & 0.00 & 2.95 & 2.96 \\
\hline & 2.5 & 0.02 & 1.00 & 0.00 & 2.99 & 2.99 & 0.00 & 1.00 & 0.00 & 2.98 & 2.98 \\
\hline \multirow[t]{8}{*}{$\# 7$} & & \multicolumn{5}{|c|}{ Physical Time Before Jump Detection } & \multicolumn{5}{|c|}{ Financial Time Before Jump Detection } \\
\hline & 0.5 & 0.01 & 1.00 & -0.00 & 2.82 & 2.94 & -0.00 & 1.00 & 0.00 & 2.77 & 2.91 \\
\hline & 1.0 & 0.01 & 1.00 & 0.00 & 2.84 & 2.97 & -0.00 & 1.00 & 0.00 & 2.82 & 2.96 \\
\hline & 2.5 & 0.02 & 1.00 & 0.00 & 2.88 & 2.99 & 0.00 & 1.00 & -0.00 & 2.90 & 2.98 \\
\hline & & \multicolumn{5}{|c|}{ Physical Time After Jump Detection } & \multicolumn{5}{|c|}{ Financial Time After Jump Detection } \\
\hline & 0.5 & 0.01 & 1.00 & -0.00 & 2.93 & 2.94 & 0.00 & 1.00 & 0.00 & 2.90 & 2.91 \\
\hline & 1.0 & 0.02 & 1.00 & -0.00 & 2.97 & 2.97 & 0.00 & 1.00 & 0.00 & 2.95 & 2.96 \\
\hline & 2.5 & 0.02 & 1.00 & 0.00 & 2.98 & 2.99 & 0.00 & 1.00 & 0.00 & 2.98 & 2.98 \\
\hline \multirow[t]{8}{*}{$\# 8$} & & \multicolumn{5}{|c|}{ Physical Time Before Jump Detection } & \multicolumn{5}{|c|}{ Financial Time Before Jump Detection } \\
\hline & 0.5 & 0.01 & 1.00 & -0.00 & 2.90 & 2.94 & 0.00 & 1.00 & 0.00 & 2.86 & 2.91 \\
\hline & 1.0 & 0.01 & 1.00 & 0.00 & 2.91 & 2.97 & 0.00 & 1.00 & 0.00 & 2.89 & 2.96 \\
\hline & 2.5 & 0.02 & 1.00 & 0.00 & 2.93 & 2.99 & 0.00 & 1.00 & 0.00 & 2.92 & 2.98 \\
\hline & & \multicolumn{5}{|c|}{ Physical Time After Jump Detection } & \multicolumn{5}{|c|}{ Financial Time After Jump Detection } \\
\hline & 0.5 & 0.01 & 1.00 & -0.00 & 2.94 & 2.94 & 0.00 & 1.00 & 0.00 & 2.91 & 2.91 \\
\hline & 1.0 & 0.02 & 1.00 & 0.00 & 2.97 & 2.97 & 0.00 & 1.00 & 0.00 & 2.95 & 2.96 \\
\hline & 2.5 & 0.03 & 1.00 & 0.00 & 2.98 & 2.99 & 0.00 & 1.00 & 0.00 & 2.98 & 2.98 \\
\hline \multirow[t]{8}{*}{$\# X 8$} & & \multicolumn{5}{|c|}{ Physical Time Before Jump Detection } & \multicolumn{5}{|c|}{ Financial Time Before Jump Detection } \\
\hline & 0.5 & 0.01 & 1.00 & -0.00 & 2.90 & 2.94 & -0.00 & 1.00 & 0.00 & 2.86 & 2.91 \\
\hline & 1.0 & 0.01 & 1.00 & 0.00 & 2.91 & 2.97 & -0.00 & 1.00 & 0.00 & 2.89 & 2.95 \\
\hline & 2.5 & 0.02 & 1.00 & 0.00 & 2.93 & 2.99 & -0.00 & 1.00 & 0.00 & 2.92 & 2.98 \\
\hline & & Physic & Time $A$ & er Jump & etectio & & Financ & Time A & er Jum & letect & \\
\hline & 0.5 & 0.01 & 1.00 & -0.00 & 2.94 & 2.94 & 0.00 & 1.00 & 0.00 & 2.91 & 2.91 \\
\hline & 1.0 & 0.02 & 1.00 & -0.00 & 2.97 & 2.97 & 0.00 & 1.00 & 0.00 & 2.95 & 2.95 \\
\hline & 2.5 & 0.02 & 1.00 & 0.00 & 2.99 & 2.99 & 0.00 & 1.00 & 0.00 & 2.98 & 2.98 \\
\hline
\end{tabular}


Table II.2A. Common Normality Tests on Simulated Datasets Without Leverage: Rejection \% for Size $5 \%$

\begin{tabular}{|c|c|c|c|c|c|c|c|c|c|c|c|}
\hline Set & Days & KS & CVM & $A D$ & $J B$ & JB Adj & KS & CVM & $A D$ & $J B$ & JB Adj \\
\hline \multirow[t]{8}{*}{$\# 1$} & & \multicolumn{5}{|c|}{ Physical Time Before Jump Detection } & \multicolumn{5}{|c|}{ Financial Time Before Jump Detection } \\
\hline & 0.5 & 5.2 & 5.1 & 5.1 & 14.8 & 3.3 & 8.5 & 7.2 & 7.8 & 30.4 & 2.9 \\
\hline & 1.0 & 6.3 & 5.2 & 5.1 & 4.9 & 3.5 & 5.5 & 5.8 & 5.8 & 6.2 & 3.5 \\
\hline & 2.5 & 5.4 & 5.3 & 5.4 & 3.6 & 3.7 & 6.0 & 6.2 & 6.9 & 4.4 & 4.7 \\
\hline & & \multicolumn{5}{|c|}{ Physical Time After Jump Detection } & \multicolumn{5}{|c|}{ Financial Time After Jump Detection } \\
\hline & 0.5 & 5.2 & 5.1 & 5.1 & 14.8 & 3.3 & 8.3 & 7.1 & 7.8 & 30.3 & 2.8 \\
\hline & 1.0 & 6.3 & 5.2 & 5.1 & 4.9 & 3.5 & 5.4 & 5.6 & 5.9 & 6.2 & 3.4 \\
\hline & 2.5 & 5.4 & 5.3 & 5.4 & 3.6 & 3.7 & 6.3 & 6.1 & 7.0 & 4.3 & 4.5 \\
\hline \multirow[t]{8}{*}{$\# 2$} & & \multicolumn{5}{|c|}{ Physical Time Before Jump Detection } & \multicolumn{5}{|c|}{ Financial Time Before Jump Detection } \\
\hline & 0.5 & 21.4 & 14.1 & 15.6 & 49.7 & 10.4 & 35.3 & 25.1 & 27.1 & 61.8 & 11.2 \\
\hline & 1.0 & 15.1 & 10.4 & 10.2 & 23.8 & 12.5 & 26.1 & 16.4 & 17.7 & 32.2 & 16.0 \\
\hline & 2.5 & 10.7 & 7.6 & 8.1 & 14.1 & 12.0 & 17.7 & 11.3 & 12.1 & 20.0 & 15.7 \\
\hline & & \multicolumn{5}{|c|}{ Physical Time After Jump Detection } & \multicolumn{5}{|c|}{ Financial Time After Jump Detection } \\
\hline & 0.5 & 6.4 & 5.5 & 5.4 & 16.4 & 3.0 & 7.3 & 6.3 & 6.9 & 33.2 & 2.7 \\
\hline & 1.0 & 5.4 & 5.3 & 5.4 & 6.0 & 4.6 & 6.5 & 5.2 & 5.4 & 7.9 & 3.6 \\
\hline & 2.5 & 5.1 & 5.6 & 5.7 & 4.7 & 4.4 & 4.3 & 5.3 & 5.7 & 4.5 & 4.5 \\
\hline \multirow[t]{8}{*}{ \#3 } & & \multicolumn{5}{|c|}{ Physical Time Before Jump Detection } & \multicolumn{5}{|c|}{ Financial Time Before Jump Detection } \\
\hline & 0.5 & 55.0 & 47.6 & 55.0 & 93.5 & 61.1 & 83.7 & 81.0 & 86.7 & 98.8 & 73.4 \\
\hline & 1.0 & 18.0 & 13.1 & 15.0 & 51.9 & 34.5 & 24.2 & 18.1 & 20.5 & 62.8 & 37.4 \\
\hline & 2.5 & 6.4 & 5.7 & 6.7 & 10.6 & 8.5 & 6.3 & 6.6 & 6.9 & 8.5 & 5.9 \\
\hline & & \multicolumn{5}{|c|}{ Physical Time After Jump Detection } & \multicolumn{5}{|c|}{ Financial Time After Jump Detection } \\
\hline & 0.5 & 6.3 & 6.4 & 7.1 & 19.2 & 3.0 & 7.8 & 7.9 & 8.9 & 39.8 & 3.1 \\
\hline & 1.0 & 5.5 & 6.3 & 6.4 & 4.7 & 3.9 & 5.5 & 6.0 & 5.7 & 6.5 & 3.4 \\
\hline & 2.5 & 5.2 & 6.0 & 6.1 & 4.4 & 4.6 & 5.5 & 6.3 & 6.8 & 3.1 & 2.5 \\
\hline \multirow[t]{8}{*}{$\# 4$} & & \multicolumn{5}{|c|}{ Physical Time Before Jump Detection } & \multicolumn{5}{|c|}{ Financial Time Before Jump Detection } \\
\hline & 0.5 & 16.4 & 10.7 & 11.6 & 48.5 & 9.7 & 29.0 & 18.6 & 20.2 & 71.5 & 8.8 \\
\hline & 1.0 & 9.2 & 7.1 & 6.6 & 15.5 & 7.6 & 13.6 & 8.8 & 9.4 & 23.7 & 8.3 \\
\hline & 2.5 & 5.4 & 4.8 & 4.8 & 7.3 & 7.0 & 5.8 & 4.8 & 5.0 & 6.9 & 5.9 \\
\hline & & \multicolumn{5}{|c|}{ Physical Time After Jump Detection } & \multicolumn{5}{|c|}{ Financial Time After Jump Detection } \\
\hline & 0.5 & 6.0 & 5.9 & 6.3 & 19.7 & 4.2 & 7.0 & 5.9 & 6.7 & 34.5 & 2.3 \\
\hline & 1.0 & 5.1 & 5.2 & 4.8 & 4.5 & 4.3 & 5.7 & 5.3 & 5.1 & 6.7 & 3.0 \\
\hline & 2.5 & 4.0 & 4.4 & 4.7 & 6.1 & 6.4 & 4.7 & 5.0 & 5.3 & 4.1 & 4.2 \\
\hline
\end{tabular}


Table II.2B. Common Normality Tests on Simulated Datasets With Leverage: Rejection \% for Size $5 \%$

\begin{tabular}{|c|c|c|c|c|c|c|c|c|c|c|c|}
\hline Set & Days & KS & CVM & $A D$ & $J B$ & JB Adj & $K S$ & CVM & $A D$ & $J B$ & JB Adj \\
\hline \multirow[t]{8}{*}{$\# 5$} & & \multicolumn{5}{|c|}{ Physical Time Before Jump Detection } & \multicolumn{5}{|c|}{ Financial Time Before Jump Detection } \\
\hline & 0.5 & 18.1 & 20.4 & 21.6 & 15.4 & 3.9 & 6.6 & 6.9 & 6.9 & 31.6 & 2.6 \\
\hline & 1.0 & 17.9 & 18.9 & 19.0 & 4.7 & 3.6 & 5.6 & 5.8 & 5.7 & 5.8 & 2.6 \\
\hline & 2.5 & 16.0 & 18.6 & 19.0 & 4.2 & 3.9 & 4.7 & 4.9 & 4.9 & 4.3 & 4.8 \\
\hline & & \multicolumn{5}{|c|}{ Physical Time After Jump Detection } & \multicolumn{5}{|c|}{ Financial Time After Jump Detection } \\
\hline & 0.5 & 18.2 & 20.4 & 21.6 & 15.5 & 3.9 & 6.5 & 6.8 & 6.8 & 31.5 & 2.5 \\
\hline & 1.0 & 17.9 & 18.8 & 19.0 & 4.7 & 3.6 & 6.0 & 5.9 & 5.8 & 5.7 & 2.7 \\
\hline & 2.5 & 16.0 & 18.6 & 19.0 & 4.2 & 3.9 & 4.6 & 5.0 & 4.9 & 4.4 & 4.8 \\
\hline \multirow[t]{8}{*}{ \#6 } & & \multicolumn{5}{|c|}{ Physical Time Before Jump Detection } & \multicolumn{5}{|c|}{ Financial Time Before Jump Detection } \\
\hline & 0.5 & 39.5 & 32.2 & 33.3 & 48.3 & 8.8 & 37.4 & 24.5 & 28.7 & 62.1 & 12.0 \\
\hline & $\mathbf{1 . 0}$ & 29.2 & 23.2 & 24.8 & 22.7 & 11.0 & 24.7 & 17.3 & 19.1 & 33.3 & 15.5 \\
\hline & 2.5 & 22.3 & 18.8 & 20.0 & 12.0 & 10.1 & 17.6 & 12.0 & 12.7 & 21.3 & 16.7 \\
\hline & & \multicolumn{5}{|c|}{ Physical Time After Jump Detection } & \multicolumn{5}{|c|}{ Financial Time After Jump Detection } \\
\hline & 0.5 & 19.0 & 20.0 & 20.5 & 16.4 & 4.1 & 8.1 & 7.3 & 7.4 & 31.7 & 2.8 \\
\hline & 1.0 & 15.3 & 16.8 & 17.3 & 5.6 & 4.6 & 5.7 & 5.6 & 5.2 & 5.5 & 2.4 \\
\hline & 2.5 & 15.1 & 17.1 & 17.7 & 4.7 & 5.2 & 4.9 & 5.1 & 4.9 & 5.3 & 5.2 \\
\hline \multirow[t]{8}{*}{$\# 7$} & & \multicolumn{5}{|c|}{ Physical Time Before Jump Detection } & \multicolumn{5}{|c|}{ Financial Time Before Jump Detection } \\
\hline & 0.5 & 60.8 & 54.9 & 60.4 & 93.2 & 61.4 & 85.1 & 83.3 & 87.7 & 99.0 & 73.0 \\
\hline & 1.0 & 26.4 & 20.1 & 22.9 & 52.7 & 34.2 & 26.6 & 17.6 & 20.5 & 63.5 & 36.3 \\
\hline & 2.5 & 11.8 & 11.4 & 12.3 & 12.0 & 9.8 & 6.7 & 6.3 & 6.5 & 8.0 & 6.3 \\
\hline & & \multicolumn{5}{|c|}{ Physical Time After Jump Detection } & \multicolumn{5}{|c|}{ Financial Time After Jump Detection } \\
\hline & 0.5 & 18.2 & 20.1 & 20.2 & 19.5 & 3.7 & 8.4 & 7.1 & 8.0 & 38.0 & 2.8 \\
\hline & 1.0 & 16.8 & 19.1 & 19.0 & 4.8 & 4.1 & 5.4 & 5.2 & 5.5 & 7.9 & 4.1 \\
\hline & 2.5 & 16.0 & 18.2 & 18.8 & 4.7 & 4.3 & 5.6 & 5.3 & 5.2 & 3.8 & 3.9 \\
\hline \multirow[t]{8}{*}{$\# 8$} & & \multicolumn{5}{|c|}{ Physical Time Before Jump Detection } & \multicolumn{5}{|c|}{ Financial Time Before Jump Detection } \\
\hline & 0.5 & 31.7 & 27.7 & 29.7 & 46.5 & 8.7 & 29.2 & 19.9 & 21.5 & 70.7 & 8.1 \\
\hline & 1.0 & 21.9 & 22.0 & 22.7 & 15.8 & 7.4 & 11.1 & 7.1 & 7.8 & 25.0 & 9.9 \\
\hline & 2.5 & 16.7 & 17.3 & 18.1 & 7.2 & 6.4 & 7.8 & 6.4 & 6.2 & 6.2 & 4.5 \\
\hline & & \multicolumn{5}{|c|}{ Physical Time After Jump Detection } & \multicolumn{5}{|c|}{ Financial Time After Jump Detection } \\
\hline & 0.5 & 19.7 & 21.8 & 22.8 & 15.3 & 4.3 & 7.8 & 6.9 & $7.7^{\circ}$ & 31.1 & 2.9 \\
\hline & 1.0 & 17.0 & 21.0 & 21.4 & 4.5 & 3.2 & 4.8 & 4.6 & 4.6 & 6.9 & 3.8 \\
\hline & 2.5 & 17.6 & 19.9 & 20.3 & 5.4 & 5.8 & 5.1 & 5.2 & 4.6 & 4.1 & 4.2 \\
\hline \multirow[t]{8}{*}{$\# X 8$} & & \multicolumn{5}{|c|}{ Physical Time Before Jump Detection } & \multicolumn{5}{|c|}{ Financial Time Before Jump Detection } \\
\hline & 0.5 & 99.9 & 99.7 & 99.9 & 100.0 & 72.4 & 100.0 & 100.0 & 100.0 & 100.0 & 84.4 \\
\hline & 1.0 & 96.9 & 96.1 & 97.1 & 95.3 & 60.8 & 90.5 & 90.7 & 94.2 & 99.5 & 72.5 \\
\hline & 2.5 & 82.1 & 84.0 & 85.4 & 46.2 & 33.7 & 18.5 & 13.8 & 15.2 & 48.9 & 28.1 \\
\hline & & Physic & Time $A t$ & Jump & etection & & Financi & Time A & er Jump & Detectic & \\
\hline & 0.5 & 92.9 & 93.5 & 95.0 & 95.9 & 2.5 & 35.3 & 30.8 & 43.3 & 100.0 & 3.1 \\
\hline & 1.0 & 86.2 & 89.2 & 91.1 & 22.4 & 4.0 & 9.2 & 7.8 & 8.5 & 43.6 & 3.6 \\
\hline & 2.5 & 82.9 & 89.2 & 89.5 & 6.0 & 4.5 & 5.4 & 6.1 & 6.2 & 5.6 & 4.0 \\
\hline
\end{tabular}


Table II.3A. N(0,1) Moment Tests on Simulated Datasets Without Leverage: Rejection \% for Size 5\%

\begin{tabular}{|c|c|c|c|c|c|c|c|c|c|c|c|c|c|}
\hline Set & Days & 1 & 2 & 3 & 4 & $3-4$ & $1-4$ & 1 & 2 & 3 & 4 & 3-4 & $1-4$ \\
\hline \multirow[t]{8}{*}{$\# 1$} & & \multicolumn{6}{|c|}{ Physical Time Before Jump Detection } & \multicolumn{6}{|c|}{ Financial Time Before Jump Detection } \\
\hline & 0.5 & 5.0 & 4.2 & 3.6 & 7.0 & 4.9 & 10.2 & 5.9 & 3.7 & 4.6 & 11.7 & 8.2 & 23.1 \\
\hline & 1.0 & 5.0 & 4.4 & 4.5 & 3.8 & 4.2 & 5.4 & 6.1 & 4.7 & 5.2 & 5.6 & 5.4 & 5.3 \\
\hline & 2.5 & 5.2 & 5.2 & 5.0 & 4.5 & 5.1 & 5.4 & 6.2 & 5.2 & 6.4 & 3.1 & 4.8 & 6.5 \\
\hline & & \multicolumn{6}{|c|}{ Physical Time After Jump Detection } & \multicolumn{6}{|c|}{ Financial Time After Jump Detection } \\
\hline & 0.5 & 5.0 & 4.3 & 3.6 & 7.0 & 4.9 & 10.2 & 6.0 & 3.8 & 4.6 & 11.3 & 8.1 & 23.1 \\
\hline & 1.0 & 5.0 & 4.4 & 4.5 & 3.8 & 4.2 & 5.4 & 6.0 & 4.6 & 5.0 & 5.8 & 5.4 & 5.2 \\
\hline & 2.5 & 5.2 & 5.2 & 5.0 & 4.5 & 5.1 & 5.4 & 6.1 & 5.3 & 6.4 & 3.4 & 5.2 & 6.5 \\
\hline \multirow[t]{8}{*}{ \#2 } & & \multicolumn{6}{|c|}{ Physical Time Before Jump Detection } & \multicolumn{6}{|c|}{ Financial Time Before Jump Detection } \\
\hline & 0.5 & 5.7 & 3.0 & 4.8 & 15.5 & 11.1 & 35.9 & 5.3 & 2.9 & 4.5 & 19.8 & 12.2 & 46.6 \\
\hline & 1.0 & 5.4 & 4.4 & 4.9 & 10.6 & 7.8 & 14.4 & 5.0 & 3.6 & 4.0 & 11.8 & 7.9 & 20.1 \\
\hline & 2.5 & 5.3 & 4.4 & 4.8 & 8.4 & 5.9 & 9.9 & 5.1 & 4.0 & 4.0 & 9.2 & 6.6 & 12.6 \\
\hline & & \multicolumn{6}{|c|}{ Physical Time After Jump Detection } & \multicolumn{6}{|c|}{ Financial Time After Jump Detection } \\
\hline & 0.5 & 5.7 & 3.9 & 5.3 & 6.1 & 6.0 & 12.0 & 5.6 & 3.5 & 4.5 & 11.4 & 7.8 & 20.6 \\
\hline & 1.0 & 5.5 & 4.1 & 4.9 & 4.9 & 4.4 & 5.9 & 5.5 & 4.4 & 4.8 & 3.9 & 4.4 & 5.9 \\
\hline & 2.5 & 5.3 & 4.9 & 5.3 & 4.5 & 5.5 & 4.8 & 5.6 & 5.8 & 5.7 & 5.3 & 5.0 & 4.7 \\
\hline \multirow[t]{8}{*}{ \#3 } & & \multicolumn{6}{|c|}{ Physical Time Before Jump Detection } & \multicolumn{6}{|c|}{ Financial Time Before Jump Detection } \\
\hline & 0.5 & 5.9 & 5.0 & 3.4 & 46.2 & 34.2 & 87.1 & 6.2 & 3.8 & 3.8 & 61.9 & 48.7 & 97.2 \\
\hline & 1.0 & 6.1 & 4.5 & 4.4 & 18.3 & 12.7 & 39.7 & 6.2 & 4.2 & 4.4 & 21.7 & 14.2 & 47.2 \\
\hline & 2.5 & 5.6 & 4.7 & 4.6 & 6.0 & 5.3 & 9.4 & 6.5 & 5.1 & 5.0 & 5.0 & 4.5 & 5.6 \\
\hline & & \multicolumn{6}{|c|}{ Physical Time After Jump Detection } & \multicolumn{6}{|c|}{ Financial Time After Jump Detection } \\
\hline & 0.5 & 5.9 & 5.7 & 4.0 & 7.4 & 5.9 & 14.8 & 6.6 & 6.0 & 4.7 & 13.6 & 11.9 & 29.6 \\
\hline & 1.0 & 6.1 & 5.4 & 4.6 & 4.6 & 4.5 & 6.5 & 6.5 & 4.8 & 4.8 & 4.8 & 4.5 & 4.5 \\
\hline & 2.5 & 6.0 & 6.0 & 5.6 & 5.0 & 5.1 & 5.1 & 6.5 & 5.0 & 3.8 & 3.7 & 3.5 & 4.1 \\
\hline \multirow[t]{8}{*}{$\# 4$} & & \multicolumn{6}{|c|}{ Physical Time Before Jump Detection } & \multicolumn{6}{|c|}{ Financial Time Before Jump Detection } \\
\hline & 0.5 & 4.4 & 4.5 & 4.5 & 17.3 & 11.5 & 36.8 & 4.3 & 3.8 & 4.2 & 24.2 & 16.6 & 55.6 \\
\hline & 1.0 & 4.4 & 5.0 & 3.9 & 7.3 & 5.5 & 11.2 & 4.2 & 4.2 & 4.2 & 8.3 & 5.9 & 16.1 \\
\hline & 2.5 & 4.5 & 4.1 & 3.9 & 4.5 & 4.3 & 5.3 & 4.2 & 4.5 & 3.8 & 4.3 & 3.7 & 5.0 \\
\hline & & \multicolumn{6}{|c|}{ Physical Time After Jump Detection } & \multicolumn{6}{|c|}{ Financial Time After Jump Detection } \\
\hline & 0.5 & 4.4 & 4.8 & 5.1 & 7.5 & 6.1 & 12.1 & 4.5 & 3.4 & 4.1 & 11.3 & 7.9 & 21.2 \\
\hline & 1.0 & 4.7 & 4.7 & 4.0 & 4.2 & 3.6 & 4.4 & 4.6 & 4.5 & 4.4 & 4.6 & 4.2 & 4.7 \\
\hline & 2.5 & 5.0 & 4.9 & 6.1 & 4.8 & 5.2 & 5.3 & 4.6 & 4.1 & 5.0 & 4.7 & 4.4 & 4.8 \\
\hline
\end{tabular}


Table II.3B. N(0,1) Moment Tests on Simulated Datasets With Leverage: Rejection \% for Size 5\%

\begin{tabular}{|c|c|c|c|c|c|c|c|c|c|c|c|c|c|}
\hline Set & Days & 1 & $\underline{2}$ & 3 & 4 & 3-4 & $1-4$ & 1 & 2 & 3 & 4 & 3-4 & $1-4$ \\
\hline \multirow[t]{8}{*}{$\# 5$} & & \multicolumn{6}{|c|}{ Physical Time Before Jump Detection } & \multicolumn{6}{|c|}{ Financial Time Before Jump Detection } \\
\hline & 0.5 & 21.1 & 4.1 & 12.2 & 8.0 & 11.9 & 21.6 & 5.4 & 4.4 & 4.4 & 12.1 & 8.1 & 20.6 \\
\hline & 1.0 & 20.6 & 3.9 & 14.8 & 5.1 & 10.6 & 13.7 & 5.5 & 4.0 & 4.0 & 5.1 & 4.1 & 5.1 \\
\hline & 2.5 & 20.4 & 4.5 & 13.7 & 3.8 & 10.4 & 12.6 & 5.6 & 4.6 & 4.3 & 4.5 & 4.4 & 4.0 \\
\hline & & \multicolumn{6}{|c|}{ Physical Time After Jump Detection } & \multicolumn{6}{|c|}{ Financial Time After Jump Detection } \\
\hline & 0.5 & 21.1 & 4.1 & 12.2 & 8.0 & 11.9 & 21.6 & 5.4 & 4.6 & 4.4 & 12.3 & 8.4 & 20.7 \\
\hline & 1.0 & 20.6 & 3.8 & 14.8 & 5.1 & 10.7 & 13.7 & 5.5 & 4.0 & 3.9 & 5.3 & 4.3 & 5.2 \\
\hline & 2.5 & 20.4 & 4.5 & 13.7 & 3.8 & 10.4 & 12.6 & 5.6 & 4.9 & 4.1 & 4.8 & 4.5 & 4.2 \\
\hline \multirow[t]{8}{*}{$\# 6$} & & \multicolumn{6}{|c|}{ Physical Time Before Jump Detection } & \multicolumn{6}{|c|}{ Financial Time Before Jump Detection } \\
\hline & 0.5 & 18.6 & 4.4 & 12.2 & 15.2 & 16.9 & 44.1 & 4.6 & 3.6 & 3.8 & 20.0 & 13.1 & 46.0 \\
\hline & 1.0 & 17.5 & 3.9 & 11.9 & 10.3 & 13.7 & 21.9 & 4.6 & 4.6 & 3.7 & 13.3 & 9.4 & 22.7 \\
\hline & 2.5 & 15.9 & 3.4 & 10.6 & 6.5 & 8.9 & 16.1 & 4.8 & 5.0 & 3.1 & 9.4 & 5.8 & 12.6 \\
\hline & & \multicolumn{6}{|c|}{ Physical Time After Jump Detection } & \multicolumn{6}{|c|}{ Financial Time After Jump Detection } \\
\hline & 0.5 & 19.2 & 4.7 & 14.1 & 6.5 & 11.3 & 20.0 & 5.4 & 4.5 & 4.8 & 11.0 & 9.0 & 22.3 \\
\hline & 1.0 & 19.8 & 4.2 & 12.7 & 4.4 & 9.7 & 12.4 & 5.5 & 3.9 & 3.4 & 4.3 & 3.7 & 4.7 \\
\hline & 2.5 & 19.4 & 6.1 & 12.9 & 5.3 & 10.3 & 10.9 & 5.4 & 3.3 & 4.8 & 4.5 & 4.6 & 4.1 \\
\hline \multirow[t]{8}{*}{$\# 7$} & & \multicolumn{6}{|c|}{ Physical Time Before Jump Detection } & \multicolumn{6}{|c|}{ Financial Time Before Jump Detection } \\
\hline & 0.5 & 14.2 & 5.5 & 9.9 & 45.6 & 39.7 & 87.2 & 5.2 & 4.0 & 3.5 & 65.6 & 50.7 & 97.0 \\
\hline & 1.0 & 13.2 & 4.2 & 8.6 & 18.8 & 17.4 & 44.0 & 4.6 & 4.2 & 3.2 & 21.2 & 13.8 & 47.8 \\
\hline & 2.5 & 11.0 & 5.0 & 8.3 & 6.3 & 8.5 & 12.3 & 4.3 & 4.8 & 4.0 & 4.7 & 3.5 & 5.6 \\
\hline & & \multicolumn{6}{|c|}{ Physical Time After Jump Detection } & \multicolumn{6}{|c|}{ Financial Time After Jump Detection } \\
\hline & 0.5 & 20.0 & 5.4 & 13.6 & 9.5 & 13.0 & 24.1 & 5.2 & 4.8 & 3.2 & 14.7 & 10.3 & 28.7 \\
\hline & 1.0 & 19.8 & 4.7 & 14.2 & 4.8 & 10.0 & 11.9 & 5.7 & 4.9 & 4.4 & 6.2 & 5.2 & 7.0 \\
\hline & 2.5 & 19.5 & 5.9 & 13.8 & 4.5 & 10.4 & 11.5 & 5.4 & 3.3 & 4.8 & 4.5 & 4.6 & 4.1 \\
\hline \multirow[t]{8}{*}{$\# 8$} & & \multicolumn{6}{|c|}{ Physical Time Before Jump Detection } & \multicolumn{6}{|c|}{ Financial Time Before Jump Detection } \\
\hline & 0.5 & 22.1 & 3.7 & 13.8 & 17.1 & 19.6 & 44.2 & 4.6 & 3.8 & 3.2 & 23.8 & 16.3 & 54.6 \\
\hline & 1.0 & 20.5 & 4.4 & 14.0 & 7.3 & 13.1 & 20.0 & 4.5 & 4.4 & 4.2 & 8.9 & 6.9 & 16.8 \\
\hline & 2.5 & 18.8 & 5.7 & 13.4 & 4.8 & 10.6 & 12.5 & 5.2 & 3.5 & 3.8 & 4.0 & 3.4 & 4.9 \\
\hline & & \multicolumn{6}{|c|}{ Physical Time After Jump Detection } & \multicolumn{6}{|c|}{ Financial Time After Jump Detection } \\
\hline & 0.5 & 23.1 & 4.4 & 14.2 & 8.5 & 13.4 & 22.6 & 5.0 & 4.3 & 4.4 & 11.0 & 8.0 & 21.8 \\
\hline & 1.0 & 22.4 & 3.7 & 15.5 & 3.1 & 11.1 & 12.4 & 4.8 & 4.8 & 4.4 & 4.6 & 4.3 & 5.1 \\
\hline & 2.5 & 21.7 & 5.4 & 16.6 & 4.8 & 12.6 & 13.6 & 5.0 & 4.8 & 5.7 & 4.0 & 4.4 & 5.2 \\
\hline \multirow[t]{8}{*}{$\# X 8$} & & \multicolumn{6}{|c|}{ Physical Time Before Jump Detection } & Finan & I Tim & Before & Jump & tectio & \\
\hline & 0.5 & 89.4 & 4.1 & 70.1 & 93.0 & 97.9 & 100.0 & 5.7 & 4.7 & 3.4 & 99.3 & 98.1 & 100.0 \\
\hline & $\mathbf{1 . 0}$ & 88.4 & 5.3 & 70.0 & 46.6 & 80.4 & 98.9 & 5.9 & 4.8 & 3.9 & 66.4 & 54.7 & 98.9 \\
\hline & 2.5 & 84.0 & 5.1 & 64.9 & 15.2 & 61.1 & 83.9 & 6.1 & 4.0 & 3.9 & 19.8 & 14.1 & 36.2 \\
\hline & & Physi & Tim & After & ump D & etectio & & Finan & I Tim & After J & Imp De & ction & \\
\hline & 0.5 & 91.9 & 4.6 & 75.2 & 46.9 & 83.7 & 99.4 & 5.5 & 5.2 & 4.0 & 83.0 & 73.0 & 99.8 \\
\hline & 1.0 & 91.5 & 5.6 & 76.1 & 9.6 & 67.6 & 86.8 & 5.4 & 5.4 & 4.2 & 15.2 & 12.4 & 34.6 \\
\hline & 2.5 & 91.3 & 5.5 & 75.6 & 4.2 & 65.3 & 80.1 & 5.6 & 5.4 & 5.3 & 6.1 & 5.0 & 5.2 \\
\hline
\end{tabular}


Table II.4A. Adjusted Moment Tests on Simulated Datasets Without Leverage: Rejection \% for Size 5\%

\begin{tabular}{|c|c|c|c|c|c|c|c|c|c|c|c|c|c|}
\hline Set & Days & 1 & $\underline{2}$ & 3 & 4 & $3-4$ & $1-4$ & 1 & $\underline{2}$ & 3 & 4 & $3-4$ & $1-4$ \\
\hline \multirow[t]{8}{*}{ \#1 } & & \multicolumn{6}{|c|}{ Physical Time Before Jump Detection } & \multicolumn{6}{|c|}{ Financial Time Before Jump Detection } \\
\hline & 0.5 & 5.0 & 5.0 & 4.1 & 5.0 & 5.4 & 5.5 & 5.9 & 4.0 & 6.1 & 4.3 & 5.6 & 5.7 \\
\hline & 1.0 & 5.0 & 4.5 & 4.8 & 4.2 & 4.9 & 4.9 & 6.1 & 5.2 & 5.5 & 5.9 & 6.3 & 5.4 \\
\hline & 2.5 & 5.2 & 5.3 & 5.3 & 5.2 & 5.5 & 5.6 & 6.2 & 5.2 & 6.6 & 3.8 & 5.6 & 6.9 \\
\hline & & \multicolumn{6}{|c|}{ Physical Time After Jump Detection } & \multicolumn{6}{|c|}{ Financial Time After Jump Detection } \\
\hline & 0.5 & 5.0 & 5.0 & 4.1 & 5.0 & 5.3 & 5.6 & 6.0 & 4.1 & 6.0 & 4.3 & 5.6 & 5.5 \\
\hline & 1.0 & 5.0 & 4.5 & 4.8 & 4.2 & 4.9 & 4.9 & 6.0 & 5.2 & 5.3 & 5.8 & 6.4 & 5.0 \\
\hline & 2.5 & 5.2 & 5.3 & 5.3 & 5.2 & 5.5 & 5.6 & 6.1 & 5.3 & 6.6 & 4.1 & 5.8 & 7.0 \\
\hline \multirow[t]{8}{*}{$\# 2$} & & \multicolumn{6}{|c|}{ Physical Time Before Jump Detection } & \multicolumn{6}{|c|}{ Financial Time Before Jump Detection } \\
\hline & 0.5 & 5.7 & 3.3 & 6.0 & 5.9 & 5.5 & 11.0 & 5.3 & 3.0 & 4.9 & 6.3 & 5.2 & 11.1 \\
\hline & 1.0 & 5.4 & 4.5 & 5.1 & 7.9 & 6.5 & 9.6 & 5.0 & 3.8 & 4.8 & 8.5 & 6.3 & 12.2 \\
\hline & 2.5 & 5.3 & 4.4 & 4.9 & 8.1 & 5.8 & 9.4 & 5.1 & 4.2 & 4.0 & 8.4 & 6.2 & 11.2 \\
\hline & & \multicolumn{6}{|c|}{ Physical Time After Jump Detection } & \multicolumn{6}{|c|}{ Financial Time After Jump Detection } \\
\hline & 0.5 & 5.7 & 4.3 & 5.9 & 3.5 & 5.2 & 5.0 & 5.6 & 3.5 & 6.0 & 3.0 & 4.7 & 4.9 \\
\hline & 1.0 & 5.5 & 4.3 & 5.4 & 3.8 & 4.9 & 5.4 & 5.5 & 4.5 & 5.3 & 5.1 & 5.0 & 5.8 \\
\hline & 2.5 & 5.3 & 5.0 & 5.4 & 4.8 & 6.0 & 5.0 & 5.6 & 6.1 & 6.0 & 5.5 & 5.3 & 5.2 \\
\hline \multirow[t]{8}{*}{$\# 3$} & & \multicolumn{6}{|c|}{ Physical Time Before Jump Detection } & \multicolumn{6}{|c|}{ Financial Time Before Jump Detection } \\
\hline & 0.5 & 5.9 & 5.4 & 3.8 & 25.8 & 17.8 & 58.5 & 6.2 & 4.4 & 4.2 & 31.6 & 22.2 & 71.0 \\
\hline & 1.0 & 6.1 & 4.7 & 4.7 & 14.3 & 10.4 & 28.3 & 6.2 & 4.8 & 4.8 & 14.4 & 9.9 & 29.9 \\
\hline & 2.5 & 5.6 & 4.7 & 4.6 & 5.6 & 5.2 & 8.5 & 6.5 & 5.1 & 5.3 & 4.9 & 4.7 & 5.3 \\
\hline & & \multicolumn{6}{|c|}{ Physical Time After Jump Detection } & \multicolumn{6}{|c|}{ Financial Time After Jump Detection } \\
\hline & 0.5 & 5.9 & 5.7 & 4.9 & 5.4 & 4.8 & 6.1 & 6.6 & 6.9 & 5.1 & 5.2 & 6.2 & 6.4 \\
\hline & 1.0 & 6.1 & 5.6 & 4.7 & 5.0 & 5.1 & 5.3 & 6.5 & 4.9 & 6.1 & 4.7 & 5.3 & 4.3 \\
\hline & 2.5 & 6.0 & 6.1 & 5.9 & 5.4 & 5.2 & 5.0 & 6.5 & 5.0 & 3.9 & 4.2 & 3.9 & 4.3 \\
\hline \multirow[t]{8}{*}{$\# 4$} & & \multicolumn{6}{|c|}{ Physical Time Before Jump Detection } & \multicolumn{6}{|c|}{ Financial Time Before Jump Detection } \\
\hline & 0.5 & 4.4 & 4.7 & 5.4 & 6.5 & 6.1 & 10.6 & 4.3 & 4.1 & 4.5 & 8.0 & 6.4 & 10.9 \\
\hline & 1.0 & 4.4 & 5.0 & 4.2 & 6.3 & 4.3 & 7.2 & 4.2 & 4.4 & 4.7 & 5.6 & 4.1 & 7.3 \\
\hline & 2.5 & 4.5 & 4.3 & 4.1 & 4.3 & 4.4 & 5.5 & 4.2 & 4.5 & 4.0 & 4.5 & 3.6 & 4.4 \\
\hline & & \multicolumn{6}{|c|}{ Physical Time After Jump Detection } & \multicolumn{6}{|c|}{ Financial Time After Jump Detection } \\
\hline & 0.5 & 4.4 & 5.1 & 6.0 & 4.3 & 4.5 & 5.3 & 4.5 & 3.7 & 5.0 & 4.7 & 3.8 & 4.0 \\
\hline & 10 & 4.7 & 4.8 & 4.1 & 5.0 & 3.8 & 3.9 & 4.6 & 4.8 & 5.3 & 4.8 & 4.7 & 4.3 \\
\hline & 2.5 & 5.0 & 5.1 & 6.1 & 5.4 & 5.7 & 5.4 & 4.6 & 4.1 & 5.0 & 5.2 & 5.0 & 4.9 \\
\hline
\end{tabular}


Table II.4B. Adjusted Moment Tests on Simulated Datasets With Leverage: Rejection \% for Size 5\%

\begin{tabular}{|c|c|c|c|c|c|c|c|c|c|c|c|c|c|}
\hline Set & Days & 1 & $\underline{2}$ & 3 & 4 & $3-4$ & $1-4$ & 1 & 2 & 3 & 4 & 3-4 & $1-4$ \\
\hline \multirow{8}{*}{$\# 5$} & & \multicolumn{6}{|c|}{ Physical Time Before Jump Detection } & \multicolumn{6}{|c|}{ Financial Time Before Jump Detection } \\
\hline & 0.5 & 21.1 & 4.6 & 13.7 & 5.1 & 11.4 & 13.4 & 5.4 & 4.9 & 5.2 & 4.9 & 5.2 & 5.1 \\
\hline & 1.0 & 20.6 & 3.9 & 15.4 & 4.9 & 11.5 & 13.4 & 5.5 & 4.4 & 4.8 & 4.9 & 5.0 & 5.0 \\
\hline & 2.5 & 20.4 & 4.5 & 14.0 & 4.3 & 11.0 & 13.0 & 5.6 & 4.9 & 4.4 & 4.2 & 4.3 & 4.6 \\
\hline & & \multicolumn{6}{|c|}{ Physical Time After Jump Detection } & \multicolumn{6}{|c|}{ Financial Time After Jump Detection } \\
\hline & 0.5 & 21.1 & 4.6 & 13.7 & 5.1 & 11.4 & 13.4 & 5.4 & 5.2 & 5.2 & 4.7 & 5.4 & 4.7 \\
\hline & 1.0 & 20.6 & 3.9 & 15.4 & 4.8 & 11.5 & 13.3 & 5.5 & 4.4 & 4.9 & 4.9 & 5.2 & 4.9 \\
\hline & 2.5 & 20.4 & 4.5 & 14.0 & 4.3 & 11.0 & 13.1 & 5.6 & 5.2 & 4.2 & 4.4 & 4.5 & 4.9 \\
\hline \multirow[t]{8}{*}{ \#6 } & & \multicolumn{6}{|c|}{ Physical Time Before Jump Detection } & \multicolumn{6}{|c|}{ Financial Time Before Jump Detection } \\
\hline & 0.5 & 18.6 & 4.4 & 13.8 & 6.2 & 12.6 & 18.2 & 4.6 & 4.0 & 4.8 & 7.9 & 6.2 & 12.4 \\
\hline & 1.0 & 17.5 & 4.1 & 12.8 & 8.0 & 11.6 & 16.4 & 4.6 & 4.9 & 4.0 & 9.7 & 6.9 & 13.8 \\
\hline & 2.5 & 15.9 & 3.6 & 10.7 & 6.5 & 9.1 & 15.1 & 4.8 & 5.2 & 3.3 & 8.1 & 5.6 & 11.5 \\
\hline & & \multicolumn{6}{|c|}{ Physical Time After Jump Detection } & \multicolumn{6}{|c|}{ Financial Time After Jump Detection } \\
\hline & 0.5 & 19.2 & 5.1 & 14.8 & 5.6 & 11.0 & 12.5 & 5.4 & 5.2 & 5.5 & 4.7 & 5.0 & 4.7 \\
\hline & 1.0 & 19.8 & 4.3 & 13.6 & 4.2 & 9.8 & 12.1 & 5.5 & 4.1 & 4.0 & 4.0 & 4.4 & 4.2 \\
\hline & 2.5 & 19.4 & 6.2 & 13.4 & 5.6 & 11.1 & 11.6 & 5.4 & 3.4 & 4.9 & 5.2 & 4.8 & 5.4 \\
\hline \multirow[t]{8}{*}{ \#7 } & & \multicolumn{6}{|c|}{ Physical Time Before Jump Detection } & \multicolumn{6}{|c|}{ Financial Time Before Jump Detection } \\
\hline & 0.5 & 14.2 & 5.7 & 10.7 & 25.4 & 25.0 & 61.3 & 5.2 & 4.3 & 4.5 & 33.6 & 24.7 & 71.6 \\
\hline & 1.0 & 13.2 & 4.7 & 9.2 & 13.8 & 14.1 & 32.9 & 4.6 & 4.5 & 3.3 & 14.3 & 10.0 & 31.1 \\
\hline & 2.5 & 11.0 & 5.0 & 8.5 & 6.1 & 8.3 & 11.8 & 4.3 & 4.8 & 4.2 & 4.6 & 3.6 & 4.9 \\
\hline & & \multicolumn{6}{|c|}{ Physical Time After Jump Detection } & \multicolumn{6}{|c|}{ Financial Time After Jump Detection } \\
\hline & 0.5 & 20.0 & 5.8 & 15.2 & 5.1 & 12.5 & 12.5 & 5.2 & 5.2 & 4.2 & 4.6 & 4.3 & 5.3 \\
\hline & $\mathbf{1 . 0}$ & 19.8 & 5.0 & 14.6 & 5.6 & 10.5 & 11.2 & 5.7 & 5.2 & 5.0 & 5.8 & 5.5 & 5.5 \\
\hline & 2.5 & 19.5 & 5.9 & 13.8 & 5.3 & 11.0 & 11.8 & 5.4 & 5.3 & 4.3 & 5.0 & 4.5 & 5.1 \\
\hline \multirow[t]{8}{*}{$\# 8$} & & \multicolumn{6}{|c|}{ Physical Time Before Jump Detection } & \multicolumn{6}{|c|}{ Financial Time Before Jump Detection } \\
\hline & 0.5 & 22.1 & 3.8 & 14.7 & 7.2 & 13.4 & 18.5 & 4.6 & 4.4 & 4.2 & 6.7 & 5.2 & 10.4 \\
\hline & $\mathbf{1 . 0}$ & 20.5 & 4.4 & 14.7 & 5.5 & 12.9 & 15.1 & 4.5 & 4.5 & 4.8 & 6.0 & 4.6 & 7.9 \\
\hline & 2.5 & 18.8 & 5.7 & 13.5 & 4.7 & 10.9 & 12.3 & 5.2 & 3.5 & 4.1 & 3.7 & 3.2 & 4.4 \\
\hline & & \multicolumn{6}{|c|}{ Physical Time After Jump Detection } & \multicolumn{6}{|c|}{ Financial Time After Jump Detection } \\
\hline & 0.5 & 23.1 & 4.8 & 15.6 & 4.7 & 12.8 & 13.7 & 5.0 & 5.1 & 5.7 & 4.8 & 5.1 & 5.0 \\
\hline & 1.0 & 22.4 & 3.9 & 16.1 & 3.8 & 11.7 & 12.8 & 4.8 & 5.0 & 4.8 & 4.7 & 4.8 & 4.7 \\
\hline & 2.5 & 21.7 & 5.5 & 16.6 & 5.5 & 13.4 & 13.8 & 5.0 & 4.9 & 6.0 & 4.3 & 5.2 & 5.3 \\
\hline \multirow[t]{8}{*}{$\# X 8$} & & \multicolumn{6}{|c|}{ Physical Time Before Jump Detection } & Finan & Time & Befor & Jump $L$ & etectio & \\
\hline & 0.5 & 33.3 & 4.6 & 22.2 & 16.1 & 26.8 & 52.8 & 5.9 & 5.8 & 4.7 & 28.1 & 21.6 & 62.1 \\
\hline & 1.0 & 31.7 & 5.6 & 20.9 & 11.8 & 23.1 & 39.4 & 5.9 & 4.6 & 4.9 & 18.7 & 14.0 & 38.5 \\
\hline & 2.5 & 31.3 & 5.5 & 21.5 & 6.4 & 17.8 & 23.3 & 5.4 & 4.7 & 4.0 & 8.5 & 6.6 & 12.2 \\
\hline & & Physi & Tim & After & ump $I$ & etection & & Finan & ITime & After. & $m p D e$ & ection & \\
\hline & 0.5 & 35.7 & 4.8 & 23.5 & 5.4 & 19.1 & 20.3 & 6.3 & 5.9 & 5.2 & 5.3 & 5.7 & 5.2 \\
\hline & 1.0 & 35.3 & 6.3 & 23.9 & 6.5 & 19.5 & 21.7 & 6.3 & 6.5 & 5.6 & 6.6 & 7.1 & 7.1 \\
\hline & 2.5 & 34.3 & 5.6 & 24.1 & 5.4 & 19.2 & 21.3 & 6.4 & 5.8 & 5.5 & 5.0 & 5.2 & 5.3 \\
\hline
\end{tabular}


Table III.1. Tests Distortion on Dataset \#8 Under Gaussian i.i.d. Microstructure Noise When RV Is Not Bias-Corrected

Descriptive Statistics

\begin{tabular}{|c|c|c|c|c|c|c|c|c|c|c|c|}
\hline Set & Days & Mean & StdD & Skew & Kurt & Kurt* $^{*}$ & Mean & StdD & Skew & Kurt & Kurt* $^{*}$ \\
\hline$\# 8 n$ & \multicolumn{6}{|c|}{ Physical Time Before Jump Detection } & \multicolumn{5}{|c|}{ Financial Time Before Jump Detection } \\
\hline & 0.5 & 0.01 & 0.97 & -0.01 & 2.91 & 2.94 & 0.00 & 0.98 & -0.00 & 2.87 & 2.92 \\
\hline & 1.0 & 0.01 & 0.97 & -0.01 & 2.92 & 2.97 & 0.00 & 0.98 & -0.00 & 2.89 & 2.96 \\
\hline & 2.5 & 0.02 & 0.97 & -0.01 & 2.93 & 2.99 & 0.00 & 0.98 & -0.00 & 2.92 & 2.98 \\
\hline & & \multicolumn{5}{|c|}{ Physical Time After Jump Detection } & \multicolumn{5}{|c|}{ Financial Time After Jump Detection } \\
\hline & 0.5 & 0.01 & 0.97 & -0.01 & 2.96 & 2.94 & 0.00 & 0.98 & -0.00 & 2.92 & 2.91 \\
\hline & 1.0 & 0.01 & 0.97 & -0.01 & 2.98 & 2.97 & 0.00 & 0.98 & -0.00 & 2.96 & 2.96 \\
\hline & 2.5 & 0.02 & 0.97 & -0.01 & 3.00 & 2.99 & 0.00 & 0.98 & -0.00 & 2.98 & 2.98 \\
\hline
\end{tabular}

\begin{tabular}{|c|c|c|c|c|c|c|c|c|c|c|c|}
\hline Set & Days & $K S$ & CVM & $A D$ & $J B$ & $J B A d j$ & $K S$ & CVM & $A D$ & $J B$ & JB Adj \\
\hline \multirow[t]{8}{*}{$\# 8 n$} & & \multicolumn{5}{|c|}{ Physical Time Before Jump Detection } & \multicolumn{5}{|c|}{ Financial Time Before Jump Detection } \\
\hline & 0.5 & 29.7 & 34.8 & 64.9 & 34.2 & 6.7 & 9.0 & 6.9 & 16.0 & 67.5 & 9.6 \\
\hline & 1.0 & 19.5 & 23.9 & 36.8 & 12.5 & 6.2 & 5.2 & 4.9 & 7.7 & 24.7 & 8.7 \\
\hline & 2.5 & 14.9 & 17.3 & 21.3 & 6.8 & 6.0 & 5.7 & 5.6 & 5.6 & 6.6 & 5.6 \\
\hline & & \multicolumn{5}{|c|}{ Physical Time After Jump Detection } & \multicolumn{5}{|c|}{ Financial Time After Jump Detection } \\
\hline & 0.5 & 50.1 & 53.7 & 75.1 & 10.0 & 6.0 & 7.9 & 8.2 & 15.5 & 25.8 & 2.7 \\
\hline & 1.0 & 37.3 & 36.7 & 50.9 & 4.1 & 4.4 & 6.4 & 7.0 & 9.0 & 6.1 & 3.8 \\
\hline & 2.5 & 23.4 & 24.6 & 31.3 & 5.6 & 5.8 & 5.7 & 5.5 & 6.3 & 3.8 & 3.5 \\
\hline
\end{tabular}

N(0,1) Moment Tests: Rejection \% for Size 5\%

\begin{tabular}{|c|c|c|c|c|c|c|c|c|c|c|c|c|c|}
\hline Set & Days & 1 & 2 & 3 & 4 & $3-4$ & $1-4$ & 1 & 2 & 3 & 4 & 3-4 & $1-4$ \\
\hline \multirow[t]{8}{*}{$\# 8 n$} & & \multicolumn{6}{|c|}{ Physical Time Before Jump Detection } & \multicolumn{6}{|c|}{ Financial Time Before Jump Detection } \\
\hline & 0.5 & 17.8 & 97.7 & 7.0 & 99.4 & 98.8 & 95.8 & 4.0 & 68.7 & 2.3 & 93.2 & 87.8 & 83.0 \\
\hline & 1.0 & 16.9 & 82.2 & 6.9 & 85.8 & 78.2 & 70.2 & 4.3 & 39.6 & 2.3 & 60.4 & 47.3 & 34.2 \\
\hline & 2.5 & 15.2 & 43.5 & 6.0 & 44.0 & 34.7 & 30.0 & 4.5 & 17.5 & 2.8 & 22.2 & 14.4 & 9.4 \\
\hline & & \multicolumn{6}{|c|}{ Physical Time After Jump Detection } & \multicolumn{6}{|c|}{ Financial Time After Jump Detection } \\
\hline & 0.5 & 18.1 & 98.2 & 6.9 & 98.4 & 96.6 & 94.0 & 4.6 & 74.8 & 3.1 & 87.4 & 77.8 & 64.7 \\
\hline & 1.0 & 18.2 & 86.7 & 7.2 & 80.0 & 71.2 & 70.8 & 4.4 & 44.8 & 3.1 & 46.2 & 34.3 & 26.1 \\
\hline & 2.5 & 18.2 & 52.3 & 7.3 & 40.1 & 31.9 & 38.1 & 4.3 & 23.3 & 3.7 & 18.6 & 12.2 & 9.6 \\
\hline
\end{tabular}

Adjusted Moment Tests: Rejection \% for Size 5\%

\begin{tabular}{|c|c|c|c|c|c|c|c|c|c|c|c|c|c|}
\hline Set & Days & 1 & $\underline{2}$ & 3 & 4 & 3-4 & $1-4$ & 1 & 2 & 3 & 4 & $3-4$ & $1-4$ \\
\hline \multirow[t]{8}{*}{$\# 8 n$} & & \multicolumn{5}{|c|}{ Physical Time Before Jump Detection } & \multicolumn{7}{|c|}{ Financial Time Before Jump Detection } \\
\hline & 0.5 & 17.8 & 97.7 & 7.4 & 97.7 & 94.8 & 93.6 & 4.0 & 70.5 & 3.2 & 78.3 & 68.9 & 53.8 \\
\hline & 1.0 & 16.9 & 82.5 & 7.1 & 82.0 & 73.5 & 67.7 & 4.3 & 40.4 & 2.8 & 50.7 & 37.4 & 25.4 \\
\hline & 2.5 & 15.2 & 43.6 & 6.3 & 42.4 & 34.0 & 30.4 & 4.5 & 17.6 & 2.9 & 20.2 & 13.7 & 9.5 \\
\hline & & \multicolumn{5}{|c|}{ Physical Time After Jump Detection } & \multicolumn{7}{|c|}{ Financial Time After Jump Detection } \\
\hline & 0.5 & 18.1 & 98.2 & 8.1 & 94.8 & 91.0 & 93.8 & 4.6 & 76.4 & 3.4 & 64.2 & 52.0 & 51.4 \\
\hline & 1.0 & 18.2 & 87.2 & 7.7 & 76.0 & 66.9 & 72.7 & 4.4 & 45.8 & 3.4 & 37.1 & 27.8 & 26.5 \\
\hline & 2.5 & 18.2 & 52.4 & 7.8 & 38.9 & 31.3 & 38.8 & 4.3 & 23.4 & 3.7 & 17.5 & 11.7 & 10.2 \\
\hline
\end{tabular}


Table III.2. Tests Success on Dataset \#8 Under Gaussian i.i.d. Microstructure Noise When RV Is Bias-Corrected

Descriptive Statistics

\begin{tabular}{cccccccccccc}
\hline Set & Days & Mean & StdD & Skew & Kurt & Kurt* & Mean & StdD & Skew & Kurt & Kurt* \\
\hline \multirow{2}{*}{ \#8n } & \multicolumn{9}{c}{ Physical Time Before Jump Detection } & \multicolumn{7}{c}{ Financial Time Before Jump Detection } \\
& $\mathbf{0 . 5}$ & 0.01 & 1.00 & 0.00 & 2.91 & 2.94 & 0.00 & 1.00 & 0.00 & 2.87 & 2.91 \\
& 1.0 & 0.01 & 1.00 & 0.00 & 2.92 & 2.97 & 0.00 & 1.00 & 0.00 & 2.89 & 2.96 \\
& 2.5 & 0.02 & 1.00 & 0.00 & 2.93 & 2.99 & 0.00 & 1.00 & 0.00 & 2.92 & 2.98
\end{tabular}

Physical Time After Jump Detection Financial Time After Jump Detection

$\begin{array}{lllllllllll}\mathbf{0 . 5} & 0.01 & 1.00 & 0.00 & 2.96 & 2.94 & 0.00 & 1.00 & 0.00 & 2.92 & 2.91 \\ 1.0 & 0.02 & 1.00 & 0.00 & 2.97 & 2.97 & 0.00 & 1.00 & 0.00 & 2.96 & 2.96 \\ 2.5 & 0.03 & 1.00 & 0.00 & 2.99 & 2.99 & 0.00 & 1.00 & 0.00 & 2.98 & 2.98\end{array}$

Common Normality Tests: Rejection \% for Size $5 \%$

\begin{tabular}{|c|c|c|c|c|c|c|c|c|c|c|c|}
\hline Set & Days & $K S$ & CVM & $A D$ & $J B$ & JB Adj & $K S$ & CVM & $A D$ & $J B$ & JB Adj \\
\hline \multirow[t]{8}{*}{$\# 8 n$} & & \multicolumn{5}{|c|}{ Physical Time Before Jump Detection } & \multicolumn{5}{|c|}{ Financial Time Before Jump Detection } \\
\hline & 0.5 & 33.5 & 29.4 & 31.1 & 35.2 & 6.8 & 30.2 & 21.3 & 23.5 & 65.5 & 7.8 \\
\hline & 1.0 & 21.8 & 22.2 & 22.9 & 14.1 & 6.8 & 11.8 & 7.7 & 7.8 & 22.0 & 7.6 \\
\hline & 2.5 & 15.7 & 17.3 & 18.4 & 6.9 & 6.1 & 6.1 & 5.3 & 5.3 & 5.7 & 3.9 \\
\hline & & \multicolumn{5}{|c|}{ Physical Time After Jump Detection } & \multicolumn{5}{|c|}{ Financial Time After Jump Detection } \\
\hline & 0.5 & 20.5 & 22.5 & 23.4 & 11.0 & 6.7 & 6.5 & 5.1 & 5.4 & 29.6 & 3.1 \\
\hline & 1.0 & 18.5 & 20.8 & 22.6 & 3.9 & 3.3 & 5.0 & 4.8 & 4.3 & 6.1 & 3.9 \\
\hline & 2.5 & 18.0 & 19.6 & 20.4 & 5.8 & 5.7 & 5.5 & 5.1 & 5.1 & 3.4 & 4.0 \\
\hline
\end{tabular}

N(0,1) Moment Tests: Rejection \% for Size 5\%

\begin{tabular}{|c|c|c|c|c|c|c|c|c|c|c|c|c|c|}
\hline Set & Days & 1 & 2 & 3 & 4 & 3-4 & $1-4$ & 1 & $\underline{2}$ & 3 & 4 & 3-4 & $1-4$ \\
\hline \multirow[t]{8}{*}{$\# 8 n$} & & \multicolumn{6}{|c|}{ Physical Time Before Jump Detection } & \multicolumn{6}{|c|}{ Financial Time Before Jump Detection } \\
\hline & 0.5 & 22.2 & 6.0 & 14.8 & 8.8 & 15.4 & 37.7 & 4.7 & 3.7 & 3.7 & 19.0 & 13.0 & 51.0 \\
\hline & 1.0 & 21.5 & 4.2 & 14.3 & 5.9 & 11.3 & 19.0 & 4.7 & 3.6 & 4.0 & 8.8 & 5.8 & 13.9 \\
\hline & 2.5 & 18.6 & 5.6 & 13.6 & 5.5 & 10.5 & 12.8 & 5.0 & 4.4 & 4.0 & 4.5 & 3.7 & 4.9 \\
\hline & & \multicolumn{6}{|c|}{ Physical Time After Jump Detection } & \multicolumn{6}{|c|}{ Financial Time After Jump Detection } \\
\hline & 0.5 & 23.3 & 6.5 & 15.6 & 5.4 & 13.3 & 21.2 & 4.6 & 4.0 & 4.6 & 8.6 & 6.0 & 20.4 \\
\hline & 1.0 & 22.5 & 3.9 & 16.3 & 3.8 & 12.4 & 12.7 & 4.6 & 4.1 & 4.2 & 4.0 & 4.5 & 4.1 \\
\hline & 2.5 & 22.1 & 6.2 & 16.8 & 5.6 & 13.8 & 12.9 & 4.7 & 4.5 & 4.8 & 4.6 & 4.8 & 4.7 \\
\hline
\end{tabular}

Adjusted Moment Tests: Rejection \% for Size 5\%

\begin{tabular}{|c|c|c|c|c|c|c|c|c|c|c|c|c|c|}
\hline Set & Days & 1 & 2 & 3 & 4 & $3-4$ & $1-4$ & 1 & $\underline{2}$ & 3 & 4 & 3-4 & $1-4$ \\
\hline \multirow[t]{8}{*}{$\# 8 n$} & & \multicolumn{6}{|c|}{ Physical Time Before Jump Detection } & \multicolumn{6}{|c|}{ Financial Time Before Jump Detection } \\
\hline & 0.5 & 22.2 & 6.8 & 16.2 & 5.4 & 13.3 & 16.7 & 4.7 & 3.9 & 4.7 & 6.1 & 5.5 & 10.5 \\
\hline & $\mathbf{1 . 0}$ & 21.5 & 4.2 & 14.8 & 4.3 & 11.5 & 14.6 & 4.7 & 3.8 & 4.6 & 5.3 & 4.4 & 7.4 \\
\hline & 2.5 & 18.6 & 5.6 & 13.7 & 5.3 & 11.1 & 12.7 & 5.0 & 4.5 & 4.1 & 4.5 & 3.9 & 4.4 \\
\hline & & \multicolumn{6}{|c|}{ Physical Time After Jump Detection } & \multicolumn{6}{|c|}{ Financial Time After Jump Detection } \\
\hline & 0.5 & 23.3 & 6.9 & 17.4 & 10.0 & 17.6 & 18.8 & 4.6 & 4.4 & 5.4 & 4.9 & 5.4 & 6.3 \\
\hline & 1.0 & 22.5 & 4.1 & 17.4 & 4.8 & 13.4 & 13.5 & 4.6 & 4.4 & 5.1 & 4.7 & 4.6 & 5.2 \\
\hline & 2.5 & 22.1 & 6.2 & 17.1 & 6.3 & 14.4 & 13.8 & 4.7 & 4.8 & 5.1 & 5.1 & 5.2 & 5.4 \\
\hline
\end{tabular}


Table III.3. Tests Power Gain on Ten Times Larger Samples of Dataset \#8 With Gaussian i.i.d. Noise When RV is Bias-Corrected

Descriptive Statistics

\begin{tabular}{cccccccccccc}
\hline Set & Days & Mean & StdD & Skew & Kurt & Kurt $^{*}$ & Mean & StdD & Skew & Kurt & Kurt $^{*}$ \\
\hline \#X8n & \multicolumn{8}{c}{ Physical Time Before Jump Detection } & \multicolumn{7}{c}{ Financial Time Before Jump Detection } \\
& $\mathbf{0 . 5}$ & 0.01 & 1.00 & 0.00 & 2.91 & 2.94 & -0.00 & 1.00 & 0.00 & 2.87 & 2.91 \\
& 1.0 & 0.01 & 1.00 & 0.00 & 2.92 & 2.97 & -0.00 & 1.00 & 0.00 & 2.89 & 2.95 \\
& 2.5 & 0.02 & 1.00 & 0.00 & 2.93 & 2.99 & -0.00 & 1.00 & 0.00 & 2.92 & 2.98 \\
& \multicolumn{8}{c}{ Physical Time After Jump Detection } & \multicolumn{6}{c}{ Financial Time After Jump Detection } \\
& $\mathbf{0 . 5}$ & 0.02 & 1.00 & 0.00 & 2.93 & 2.99 & 0.00 & 1.00 & 0.00 & 2.91 & 2.91 \\
& 1.0 & 0.01 & 1.00 & 0.00 & 2.95 & 2.94 & 0.00 & 1.00 & 0.00 & 2.96 & 2.95 \\
& $\mathbf{2 . 5}$ & 0.02 & 1.00 & 0.00 & 2.98 & 2.97 & 0.00 & 1.00 & 0.00 & 2.98 & 2.98
\end{tabular}

Common Normality Tests: Rejection \% for Size 5\%

\begin{tabular}{cccccccccccc}
\hline Set & Days & KS & CVM & AD & JB & JB Adj & KS & CVM & AD & JB & JB Adj \\
\hline \#X8n & \multicolumn{9}{c}{ Physical Time Before Jump Detection } & \multicolumn{7}{c}{ Financial Time Before Jump Detection } \\
& $\mathbf{0 . 5}$ & 99.9 & 99.7 & 99.9 & 100.0 & 33.1 & 100.0 & 100.0 & 100.0 & 100.0 & 77.6 \\
& 1.0 & 97.2 & 96.2 & 97.3 & 91.2 & 48.5 & 89.3 & 88.6 & 92.8 & 99.2 & 70.2 \\
& 2.5 & 82.4 & 83.8 & 85.5 & 43.2 & 30.9 & 19.2 & 13.5 & 14.8 & 48.2 & 28.5 \\
& \multicolumn{8}{c}{ Physical Time After Jump Detection } & \multicolumn{5}{c}{ Financial Time After Jump Detection } \\
& $\mathbf{0 . 5}$ & 94.2 & 94.6 & 95.8 & 76.3 & 13.7 & 36.6 & 32.5 & 43.6 & 99.8 & 3.3 \\
& 1.0 & 85.8 & 89.1 & 91.4 & 13.5 & 5.9 & 8.1 & 7.2 & 8.6 & 39.7 & 3.2 \\
& 2.5 & 82.1 & 89.3 & 89.6 & 6.1 & 4.9 & 4.9 & 5.7 & 5.6 & 8.1 & 5.2
\end{tabular}

N(0,1) Moment Tests: Rejection \% for Size 5\%

\begin{tabular}{|c|c|c|c|c|c|c|c|c|c|c|c|c|c|}
\hline Set & Days & 1 & $\underline{2}$ & 3 & 4 & $3-4$ & $1-4$ & 1 & $\underline{2}$ & 3 & 4 & $3-4$ & $1-4$ \\
\hline \multirow{8}{*}{ \#X8n } & & \multicolumn{6}{|c|}{ Physical Time Before Jump Detection } & \multicolumn{6}{|c|}{ Financial Time Before Jump Detection } \\
\hline & 0.5 & 89.6 & 18.0 & 75.8 & 49.3 & 84.9 & 100.0 & 6.0 & 6.2 & 4.0 & 97.6 & 97.0 & 100.0 \\
\hline & 1.0 & 88.4 & 6.9 & 72.4 & 29.6 & 75.0 & 98.3 & 6.0 & 4.6 & 3.7 & 64.3 & 51.3 & 98.1 \\
\hline & 2.5 & 84.3 & 6.3 & 65.4 & 12.7 & 60.2 & 82.6 & 6.1 & 4.1 & 4.6 & 18.5 & 12.9 & 36.2 \\
\hline & & \multicolumn{6}{|c|}{ Physical Time After Jump Detection } & \multicolumn{6}{|c|}{ Financial Time After Jump Detection } \\
\hline & 0.5 & 92.1 & 21.1 & 78.9 & 8.5 & 71.4 & 97.7 & 5.3 & 6.7 & 4.0 & 71.9 & 59.9 & 99.5 \\
\hline & 1.0 & 92.0 & 8.0 & 77.7 & 5.8 & 68.6 & 84.7 & 5.2 & 4.5 & 5.1 & 12.9 & 9.7 & 29.4 \\
\hline & 2.5 & 91.9 & 7.4 & 76.2 & 4.8 & 66.1 & 80.0 & 5.6 & 4.8 & 6.2 & 5.3 & 4.4 & 6.2 \\
\hline
\end{tabular}

Adjusted Moment Tests: Rejection \% for Size 5\%

\begin{tabular}{|c|c|c|c|c|c|c|c|c|c|c|c|c|c|}
\hline Set & Days & 1 & 2 & 3 & 4 & $3-4$ & $1-4$ & 1 & 2 & 3 & $\overline{4}$ & 3-4 & $1-4$ \\
\hline \multirow[t]{8}{*}{$\# X 8 n$} & & \multicolumn{6}{|c|}{ Physical Time Before Jump Detection } & \multicolumn{6}{|c|}{ Financial Time Before Jump Detection } \\
\hline & 0.5 & 89.6 & 19.0 & 77.6 & 5.3 & 67.3 & 90.8 & 6.0 & 6.3 & 4.8 & 31.3 & 22.7 & 78.0 \\
\hline & 1.0 & 88.4 & 7.0 & 73.4 & 11.9 & 66.7 & 89.6 & 6.0 & 5.2 & 4.4 & 26.8 & 19.2 & 64.9 \\
\hline & 2.5 & 84.3 & 6.4 & 65.8 & 10.3 & 59.2 & 79.3 & 6.1 & 4.2 & 4.9 & 11.5 & 9.2 & 20.9 \\
\hline & & \multicolumn{6}{|c|}{ Physical Time After Jump Detection } & \multicolumn{6}{|c|}{ Financial Time After Jump Detection } \\
\hline & 0.5 & 92.1 & 22.1 & 81.3 & 36.6 & 81.5 & 87.9 & 5.3 & 7.5 & 5.3 & 7.3 & 6.7 & 6.6 \\
\hline & 1.0 & 92.0 & 8.2 & 78.9 & 11.3 & 73.1 & 80.5 & 5.2 & 4.9 & 5.5 & 4.9 & 5.6 & 5.8 \\
\hline & 2.5 & 91.9 & 7.4 & 76.3 & 6.5 & 67.3 & 79.8 & 5.6 & 4.9 & 6.2 & 5.1 & 5.2 & 5.1 \\
\hline
\end{tabular}


Table IV.1. Test P-Values on The S\&P500 Futures Dataset When RV is Not Bias-Corrected Descriptive Statistics

\begin{tabular}{ccccccccccccc}
\hline Set & Days & Mean & StdD & Skew & Kurt & Kurt* & Mean & StdD & Skew & Kurt & Kurt* \\
\hline SP500 & \multicolumn{8}{c}{ Physical Time Before Jump Detection } & \multicolumn{7}{c}{ Financial Time Before Jump Detection } \\
& $\mathbf{0 . 5}$ & 0.04 & 0.93 & 0.03 & 2.84 & 2.94 & 0.01 & 0.99 & -0.00 & 2.73 & 2.86 \\
& 1.0 & 0.05 & 0.98 & 0.11 & 2.92 & 2.97 & 0.01 & 0.99 & 0.00 & 2.72 & 2.92 \\
& 2.5 & 0.07 & 0.94 & -0.01 & 2.92 & 2.99 & 0.01 & 0.98 & -0.03 & 2.82 & 2.96 \\
& \multicolumn{8}{c}{ Physical Time After Jump Detection } & \multicolumn{7}{c}{ Financial Time After Jump Detection } \\
& $\mathbf{0 . 5}$ & 0.04 & 0.93 & 0.02 & 2.87 & 2.94 & 0.01 & 0.98 & 0.04 & 2.70 & 2.86 \\
& 1.0 & 0.06 & 0.97 & 0.11 & 2.94 & 2.97 & 0.01 & 1.00 & 0.05 & 2.76 & 2.93 \\
& $\mathbf{2 . 5}$ & 0.07 & 0.94 & 0.00 & 2.94 & 2.99 & 0.01 & 0.98 & 0.04 & 2.81 & 2.97
\end{tabular}

Common Normality Tests: P-Values

\begin{tabular}{|c|c|c|c|c|c|c|c|c|c|c|c|}
\hline Set & Days & $K S$ & CVM & $A D$ & $J B$ & JB Adj & $K S$ & CVM & $A D$ & $J B$ & JB Adj \\
\hline \multirow[t]{8}{*}{ SP500 } & & \multicolumn{5}{|c|}{ Physical Time Before Jump Detection } & \multicolumn{5}{|c|}{ Financial Time Before Jump Detection } \\
\hline & 0.5 & 0.000 & 0.000 & 0.000 & 0.008 & 0.536 & 0.125 & 0.271 & 0.194 & 0.000 & 0.530 \\
\hline & 1.0 & 0.023 & 0.013 & 0.002 & 0.006 & 0.155 & 0.511 & 0.482 & 0.336 & 0.002 & 0.449 \\
\hline & 2.5 & 0.016 & 0.010 & 0.003 & 0.779 & 0.955 & 0.769 & 0.817 & 0.811 & 0.298 & 0.814 \\
\hline & & \multicolumn{5}{|c|}{ Physical Time After Jump Detection } & \multicolumn{5}{|c|}{ Financial Time After Jump Detection } \\
\hline & 0.5 & 0.000 & 0.000 & 0.000 & 0.045 & 0.735 & 0.441 & 0.271 & 0.074 & 0.000 & 0.204 \\
\hline & 1.0 & 0.011 & 0.007 & 0.001 & 0.013 & 0.194 & 0.277 & 0.314 & 0.215 & 0.004 & 0.402 \\
\hline & 2.5 & 0.007 & 0.005 & 0.002 & 0.874 & 0.979 & 0.953 & 0.897 & 0.751 & 0.220 & 0.725 \\
\hline
\end{tabular}

N(0,1) Moment Tests: P-Values

\begin{tabular}{|c|c|c|c|c|c|c|c|c|c|c|c|c|c|}
\hline Set & Days & 1 & 2 & 3 & 4 & 3-4 & $1-4$ & 1 & 2 & 3 & 4 & 3-4 & $1-4$ \\
\hline \multirow[t]{8}{*}{ SP500 } & & \multicolumn{6}{|c|}{ Physical Time Before Jump Detection } & \multicolumn{6}{|c|}{ Financial Time Before Jump Detection } \\
\hline & 0.5 & 0.000 & 0.000 & 0.001 & 0.000 & 0.000 & 0.000 & 0.537 & 0.144 & 0.687 & 0.000 & 0.002 & 0.000 \\
\hline & 1.0 & 0.001 & 0.044 & 0.000 & 0.040 & 0.000 & 0.000 & 0.660 & 0.187 & 0.709 & 0.005 & 0.019 & 0.013 \\
\hline & 2.5 & 0.009 & 0.001 & 0.080 & 0.003 & 0.003 & 0.001 & 0.666 & 0.231 & 0.941 & 0.090 & 0.238 & 0.476 \\
\hline & & \multicolumn{6}{|c|}{ Physical Time After Jump Detection } & \multicolumn{6}{|c|}{ Financial Time After Jump Detection } \\
\hline & 0.5 & 0.000 & 0.000 & 0.002 & 0.000 & 0.000 & 0.000 & 0.486 & 0.006 & 0.153 & 0.000 & 0.000 & 0.000 \\
\hline & 1.0 & 0.000 & 0.023 & 0.000 & 0.033 & 0.000 & 0.000 & 0.538 & 0.713 & 0.196 & 0.069 & 0.083 & 0.025 \\
\hline & 2.5 & 0.005 & 0.002 & 0.054 & 0.006 & 0.003 & 0.001 & 0.570 & 0.254 & 0.400 & 0.093 & 0.172 & 0.415 \\
\hline
\end{tabular}

Adjusted Moment Tests: P-Values

\begin{tabular}{cccccccccccccc}
\hline Set & Days & 1 & 2 & 3 & 4 & $3-4$ & $1-4$ & 1 & 2 & 3 & 4 & $3-4$ & $1-4$ \\
\hline SP500 & \multicolumn{8}{c}{ Physical Time Before Jump Detection } & \multicolumn{6}{c}{ Financial Time Before Jump Detection } \\
& $\mathbf{0 . 5}$ & 0.000 & 0.000 & 0.001 & 0.000 & 0.000 & 0.000 & 0.537 & 0.130 & 0.667 & 0.011 & 0.035 & 0.059 \\
& $\mathbf{1 . 0}$ & 0.001 & 0.042 & 0.000 & 0.055 & 0.000 & 0.000 & 0.660 & 0.178 & 0.699 & 0.015 & 0.047 & 0.070 \\
& 2.5 & 0.009 & 0.001 & 0.078 & 0.003 & 0.003 & 0.001 & 0.666 & 0.226 & 0.940 & 0.114 & 0.286 & 0.564 \\
& \multicolumn{8}{c}{ Physical Time } & After Jump Detection & \multicolumn{6}{c}{ Financial Time After Jump Detection } \\
& $\mathbf{0 . 5}$ & 0.000 & 0.000 & 0.001 & 0.000 & 0.000 & 0.000 & 0.486 & 0.004 & 0.127 & 0.000 & 0.000 & 0.000 \\
& $\mathbf{1 . 0}$ & 0.000 & 0.022 & 0.000 & 0.046 & 0.000 & 0.000 & 0.538 & 0.707 & 0.181 & 0.152 & 0.146 & 0.112 \\
& 2.5 & 0.005 & 0.002 & 0.052 & 0.006 & 0.004 & 0.001 & 0.570 & 0.251 & 0.393 & 0.110 & 0.194 & 0.472 \\
\hline
\end{tabular}


Table IV.2. Tests P-Values on The S\&P500 Futures Dataset When RV is Bias-Corrected Descriptive Statistics

\begin{tabular}{cccccccccccc}
\hline Set & Days & Mean & StdD & Skew & Kurt & Kurt* & Mean & StdD & Skew & Kurt & Kurt* \\
\hline SP500 & \multicolumn{8}{c}{ Physical Time Before Jump Detection } & \multicolumn{7}{c}{ Financial Time Before Jump Detection } \\
& $\mathbf{0 . 5}$ & 0.05 & 0.95 & 0.03 & 2.83 & 2.94 & 0.01 & 0.99 & 0.02 & 2.64 & 2.86 \\
& 1.0 & 0.06 & 1.00 & 0.12 & 2.90 & 2.97 & 0.01 & 1.00 & 0.02 & 2.86 & 2.92 \\
& 2.5 & 0.07 & 0.95 & 0.01 & 2.91 & 2.99 & 0.01 & 1.01 & 0.00 & 2.99 & 2.96 \\
& \multicolumn{8}{c}{ Physical Time } & After Jump Detection & \multicolumn{7}{c}{ Financial Time After Jump Detection } \\
& $\mathbf{0 . 5}$ & 0.05 & 0.95 & 0.03 & 2.85 & 2.94 & 0.01 & 0.99 & 0.02 & 2.74 & 2.86 \\
& $\mathbf{1 . 0}$ & 0.06 & 0.99 & 0.12 & 2.93 & 2.97 & 0.01 & 1.00 & -0.03 & 2.81 & 2.92 \\
& $\mathbf{2 . 5}$ & 0.07 & 0.96 & 0.01 & 2.94 & 2.99 & 0.01 & 1.00 & 0.04 & 3.03 & 2.97
\end{tabular}

Common Normality Tests: P-Values

\begin{tabular}{|c|c|c|c|c|c|c|c|c|c|c|c|}
\hline Set & Days & $K S$ & CVM & $A D$ & $J B$ & JB Adj & $K S$ & CVM & $A D$ & $J B$ & JB Adj \\
\hline SP500 & & \multicolumn{5}{|c|}{ Physical Time Before Jump Detection } & \multicolumn{5}{|c|}{ Financial Time Before Jump Detection } \\
\hline & 0.5 & 0.000 & 0.000 & 0.000 & 0.002 & 0.414 & 0.162 & 0.082 & 0.043 & 0.000 & 0.138 \\
\hline & $\mathbf{1 . 0}$ & 0.071 & 0.014 & 0.001 & 0.003 & 0.121 & 0.109 & 0.355 & 0.344 & 0.176 & 0.899 \\
\hline & 2.5 & 0.029 & 0.012 & 0.004 & 0.755 & 0.948 & 0.618 & 0.505 & 0.583 & 0.990 & 0.993 \\
\hline & & \multicolumn{5}{|c|}{ Physical Time After Jump Detection } & \multicolumn{5}{|c|}{ Financial Time After Jump Detection } \\
\hline & 0.5 & 0.000 & 0.000 & 0.000 & 0.015 & 0.599 & 0.317 & 0.389 & 0.184 & 0.000 & 0.507 \\
\hline & 1.0 & 0.036 & 0.008 & 0.001 & 0.006 & 0.154 & 0.310 & 0.518 & 0.480 & 0.035 & 0.687 \\
\hline & 2.5 & 0.017 & 0.006 & 0.003 & 0.851 & 0.969 & 0.560 & 0.595 & 0.694 & 0.770 & 0.885 \\
\hline
\end{tabular}

N(0,1) Moment Tests: P-Values

\begin{tabular}{cccccccccccccc}
\hline Set & Days & 1 & 2 & 3 & 4 & $3-4$ & $1-4$ & 1 & 2 & 3 & 4 & $3-4$ & $1-4$ \\
\hline SP500 & \multicolumn{8}{c}{ Physical Time Before Jump Detection } & \multicolumn{6}{c}{ Financial Time Before Jump Detection } \\
& $\mathbf{0 . 5}$ & 0.000 & 0.000 & 0.000 & 0.000 & 0.000 & 0.000 & 0.544 & 0.272 & 0.394 & 0.000 & 0.000 & 0.000 \\
& $\mathbf{1 . 0}$ & 0.000 & 0.559 & 0.000 & 0.345 & 0.000 & 0.000 & 0.647 & 0.750 & 0.552 & 0.245 & 0.427 & 0.464 \\
& 2.5 & 0.006 & 0.014 & 0.046 & 0.020 & 0.009 & 0.008 & 0.661 & 0.497 & 0.694 & 0.584 & 0.796 & 0.955 \\
& \multicolumn{8}{c}{ Physical Time After Jump Detection } & \multicolumn{6}{c}{ Financial Time After Jump Detection } \\
& $\mathbf{0 . 5}$ & 0.000 & 0.000 & 0.001 & 0.000 & 0.000 & 0.000 & 0.506 & 0.184 & 0.393 & 0.001 & 0.002 & 0.000 \\
& 1.0 & 0.000 & 0.412 & 0.000 & 0.315 & 0.000 & 0.000 & 0.520 & 0.478 & 0.942 & 0.069 & 0.191 & 0.128 \\
& 2.5 & 0.003 & 0.024 & 0.028 & 0.038 & 0.010 & 0.008 & 0.566 & 0.831 & 0.379 & 0.944 & 0.678 & 0.925
\end{tabular}

Adjusted Moment Tests: P-Values

\begin{tabular}{cccccccccccccc}
\hline Set & Days & 1 & 2 & 3 & 4 & $3-4$ & $1-4$ & 1 & 2 & 3 & 4 & $3-4$ & $1-4$ \\
\hline SP500 & \multicolumn{8}{c}{ Physical Time Before Jump Detection } & \multicolumn{7}{c}{ Financial Time Before Jump Detection } \\
& $\mathbf{0 . 5}$ & 0.000 & 0.000 & 0.000 & 0.000 & 0.000 & 0.000 & 0.544 & 0.254 & 0.363 & 0.002 & 0.005 & 0.000 \\
& 1.0 & 0.000 & 0.556 & 0.000 & 0.441 & 0.000 & 0.000 & 0.647 & 0.744 & 0.538 & 0.491 & 0.653 & 0.884 \\
& 2.5 & 0.006 & 0.013 & 0.045 & 0.021 & 0.009 & 0.008 & 0.661 & 0.492 & 0.688 & 0.460 & 0.703 & 0.944 \\
& \multicolumn{8}{c}{ Physical Time After Jump Detection } & \multicolumn{6}{c}{ Financial Time After Jump Detection } \\
& $\mathbf{0 . 5}$ & 0.000 & 0.000 & 0.000 & 0.000 & 0.000 & 0.000 & 0.506 & 0.168 & 0.362 & 0.015 & 0.035 & 0.060 \\
& 1.0 & 0.000 & 0.556 & 0.000 & 0.441 & 0.000 & 0.000 & 0.520 & 0.469 & 0.940 & 0.156 & 0.365 & 0.407 \\
& $\mathbf{2 . 5}$ & 0.006 & 0.013 & 0.045 & 0.021 & 0.009 & 0.008 & 0.566 & 0.830 & 0.372 & 0.951 & 0.670 & 0.889 \\
\hline
\end{tabular}


Table IV.3. Tests P-Values on The S\&P500 Futures Dataset When RV is Bias-Corrected and The Jump Extraction Procedure Is Adjusted for the Intraday Volatility Pattern Descriptive Statistics

\begin{tabular}{cccccccccccc}
\hline Set & Days & Mean & StdD & Skew & Kurt & Kurt & Mean & StdD & Skew & Kurt & Kurt \\
\hline SP500 & \multicolumn{8}{c}{ Physical Time Before Jump Detection } & \multicolumn{3}{c}{ Financial Time Before Jump Detection } \\
& $\mathbf{0 . 5}$ & 0.046 & 0.947 & 0.034 & 2.827 & 2.94 & 0.007 & 0.991 & 0.018 & 2.638 & 2.86 \\
& $\mathbf{1 . 0}$ & 0.056 & 0.992 & 0.121 & 2.903 & 2.97 & 0.007 & 0.996 & 0.015 & 2.859 & 2.92 \\
& $\mathbf{2 . 5}$ & 0.067 & 0.954 & 0.006 & 2.914 & 2.99 & 0.011 & 1.012 & 0.004 & 2.989 & 2.96 \\
& \multicolumn{8}{c}{ Physical Time After Jump Detection } & \multicolumn{8}{c}{ Financial Time After Jump Detection } \\
& $\mathbf{0 . 5}$ & 0.047 & 0.946 & 0.027 & 2.857 & 2.94 & 0.008 & 0.990 & 0.028 & 2.760 & 2.86 \\
& $\mathbf{1 . 0}$ & 0.059 & 0.990 & 0.116 & 2.937 & 2.97 & 0.010 & 0.992 & 0.009 & 2.819 & 2.92 \\
& $\mathbf{2 . 5}$ & 0.074 & 0.957 & 0.015 & 2.934 & 2.99 & 0.016 & 0.994 & 0.030 & 2.837 & 2.97
\end{tabular}

Common Normality Tests: P-Values

\begin{tabular}{cccccccccccc}
\hline Set & Days & $K S$ & $C V M$ & $A D$ & $J B$ & $J B A d j$ & $K S$ & $C V M$ & $A D$ & JB & JB Adj \\
\hline SP500 & \multicolumn{8}{c}{ Physical Time Before Jump Detection } & \multicolumn{7}{c}{ Financial Time Before Jump Detection } \\
& $\mathbf{0 . 5}$ & 0.000 & 0.000 & 0.000 & 0.002 & 0.414 & 0.162 & 0.082 & 0.043 & 0.000 & 0.138 \\
& 1.0 & 0.071 & 0.014 & 0.001 & 0.003 & 0.121 & 0.109 & 0.355 & 0.344 & 0.176 & 0.899 \\
& 2.5 & 0.029 & 0.012 & 0.004 & 0.755 & 0.948 & 0.618 & 0.505 & 0.583 & 0.990 & 0.993 \\
& \multicolumn{8}{c}{ Physical Time After Jump Detection } & Financial Time After Jump Detection \\
& $\mathbf{0 . 5}$ & 0.000 & 0.000 & 0.000 & 0.016 & 0.601 & 0.460 & 0.346 & 0.212 & 0.000 & 0.548 \\
& 1.0 & 0.033 & 0.007 & 0.001 & 0.007 & 0.152 & 0.642 & 0.734 & 0.716 & 0.060 & 0.784 \\
& 2.5 & 0.011 & 0.005 & 0.002 & 0.819 & 0.959 & 0.808 & 0.724 & 0.713 & 0.344 & 0.816 \\
\end{tabular}

\begin{tabular}{cccccccccccccc}
\hline Set & Days & 1 & 2 & 3 & 4 & $3-4$ & $1-4$ & 1 & 2 & 3 & 4 & $3-4$ & $1-4$ \\
\hline SP500 & \multicolumn{8}{c}{ Physical Time Before Jump Detection } & \multicolumn{6}{c}{ Financial Time Before Jump Detection } \\
& $\mathbf{0 . 5}$ & 0.000 & 0.000 & 0.000 & 0.000 & 0.000 & 0.000 & 0.544 & 0.272 & 0.394 & 0.000 & 0.000 & 0.000 \\
& 1.0 & 0.000 & 0.559 & 0.000 & 0.345 & 0.000 & 0.000 & 0.647 & 0.750 & 0.552 & 0.245 & 0.427 & 0.464 \\
& 2.5 & 0.006 & 0.014 & 0.046 & 0.020 & 0.009 & 0.008 & 0.661 & 0.497 & 0.694 & 0.584 & 0.796 & 0.955 \\
& \multicolumn{8}{c}{ Physical Time After Jump Detection } & \multicolumn{6}{c}{ Financial Time After Jump Detection } \\
& $\mathbf{0 . 5}$ & 0.000 & 0.000 & 0.000 & 0.000 & 0.000 & 0.000 & 0.461 & 0.192 & 0.235 & 0.001 & 0.003 & 0.000 \\
& 1.0 & 0.000 & 0.463 & 0.000 & 0.393 & 0.000 & 0.000 & 0.526 & 0.477 & 0.527 & 0.081 & 0.179 & 0.196 \\
& 2.5 & 0.003 & 0.021 & 0.023 & 0.033 & 0.008 & 0.006 & 0.525 & 0.726 & 0.428 & 0.342 & 0.465 & 0.658
\end{tabular}

Adjusted Moment Tests: P-Values

\begin{tabular}{cccccccccccccc}
\hline Set & Days & 1 & 2 & 3 & 4 & $3-4$ & $1-4$ & 1 & 2 & 3 & 4 & $3-4$ & $1-4$ \\
\hline SP500 & \multicolumn{8}{c}{ Physical Time Before Jump Detection } & \multicolumn{1}{c}{ Financial Time Before Jump Detection } \\
& $\mathbf{0 . 5}$ & 0.000 & 0.000 & 0.000 & 0.000 & 0.000 & 0.000 & 0.544 & 0.254 & 0.363 & 0.002 & 0.005 & 0.000 \\
& 1.0 & 0.000 & 0.556 & 0.000 & 0.441 & 0.000 & 0.000 & 0.647 & 0.744 & 0.538 & 0.491 & 0.653 & 0.884 \\
& 2.5 & 0.006 & 0.013 & 0.045 & 0.021 & 0.009 & 0.008 & 0.661 & 0.492 & 0.688 & 0.460 & 0.703 & 0.944 \\
& \multicolumn{8}{c}{ Physical Time } & After Jump Detection & \multicolumn{1}{c}{ Financial Time After Jump Detection } \\
& $\mathbf{0 . 5}$ & 0.000 & 0.000 & 0.000 & 0.000 & 0.000 & 0.000 & 0.461 & 0.176 & 0.205 & 0.032 & 0.044 & 0.108 \\
& 1.0 & 0.000 & 0.460 & 0.000 & 0.499 & 0.000 & 0.000 & 0.526 & 0.468 & 0.512 & 0.182 & 0.331 & 0.551 \\
& 2.5 & 0.003 & 0.021 & 0.022 & 0.035 & 0.008 & 0.006 & 0.525 & 0.724 & 0.421 & 0.398 & 0.506 & 0.744 \\
\hline
\end{tabular}


Table V.1. S\&P 500 Day Length Variation in Financial Time Before Jump Detection

\begin{tabular}{|c|c|c|c|}
\hline & $\begin{array}{l}\text { Day } \\
\text { Count }\end{array}$ & $\begin{array}{l}\text { Day Length in Phys. } \\
\text { Time Mean x } 2 \text { min }\end{array}$ & $\begin{array}{l}\text { Day Length in Fin. Time } \\
\text { Mean }(\text { StdD }) \times 10^{\wedge}-5\end{array}$ \\
\hline Days in Physical Time: & 4126 & 195.00 & $8.97(11.83)$ \\
\hline Corresponding Days in Feasible Financial Time: & 3923 & 205.09 & $9.43(3.65)$ \\
\hline - of physical time length from 1 to $5 \times 2 \mathrm{~min}$ & 32 & 2.63 & $23.05(31.41)$ \\
\hline - of physical time length from 6 to $15 \times 2 \mathrm{~min}$ & 84 & 10.81 & $12.16(7.94)$ \\
\hline - of physical time length from 16 to $30 \times 2 \mathrm{~min}$ & 202 & 23.27 & $10.21(4.28)$ \\
\hline - of physical time length from 31 to $60 \times 2 \mathrm{~min}$ & 450 & 45.45 & $9.53(2.31)$ \\
\hline - of physical time length from 61 to $195 \times 2 \mathrm{~min}$ & 1652 & 123.72 & $9.24(1.29)$ \\
\hline - of physical time length from 196 to $390 \times 2 \mathrm{~min}$ & 979 & 273.47 & $9.08(0.39)$ \\
\hline - of physical time length from 391 to $780 \times 2 \mathrm{~min}$ & 453 & 528.54 & $9.07(1.16)$ \\
\hline - of physical time length from 781 to $1,666 \times 2 \mathrm{~min}$ & 71 & 941.89 & $9.02(1.29)$ \\
\hline
\end{tabular}

Table V.2. S\&P 500 Day Length Variation in Financial Time After Jump Detection

\begin{tabular}{|c|c|c|c|}
\hline & $\begin{array}{l}\text { Day } \\
\text { Count }\end{array}$ & $\begin{array}{l}\text { Day Length in Phys. Time } \\
\text { Mean x } 2 \text { min }\end{array}$ & $\begin{array}{l}\text { Day Length in Fin. Time } \\
\text { Mean (StdD) } \times 10^{\wedge}-5\end{array}$ \\
\hline Days in Physical Time: & 4126 & 195 & $8.57(9.84)$ \\
\hline Corresponding Days in Feasible Financial Time: & 4022 & 200.04 & $8.79(0.73)$ \\
\hline - of physical time length from 1 to $5 \times 2 \mathrm{~min}$ & 22 & 3.72 & $11.93(5.37)$ \\
\hline - of physical time length from 6 to $15 \times 2 \mathrm{~min}$ & 87 & 11.39 & $9.82(1.67)$ \\
\hline - of physical time length from 16 to $30 \times 2 \mathrm{~min}$ & 197 & 23.27 & $9.20(1.10)$ \\
\hline - of physical time length from 31 to $60 \times 2 \mathrm{~min}$ & 502 & 45.86 & $8.94(0.71)$ \\
\hline - of physical time length from 61 to $195 \times 2 \mathrm{~min}$ & 1714 & 122.99 & $8.75(0.48)$ \\
\hline - of physical time length from 196 to $390 \times 2 \mathrm{~min}$ & 987 & 272.28 & $8.65(0.13)$ \\
\hline - of physical time length from 391 to $780 \times 2 \mathrm{~min}$ & 446 & 522.47 & $8.62(0.08)$ \\
\hline - of physical time length from 781 to $1,750 \times 2 \mathrm{~min}$ & 67 & 944.99 & $8.61(0.04)$ \\
\hline
\end{tabular}


Figure I. $\quad$ Log-PDF Plot of the Finite Sample Distribution (solid line) for Different $n_{t}$ against Standard Normal Distribution (dashed line)
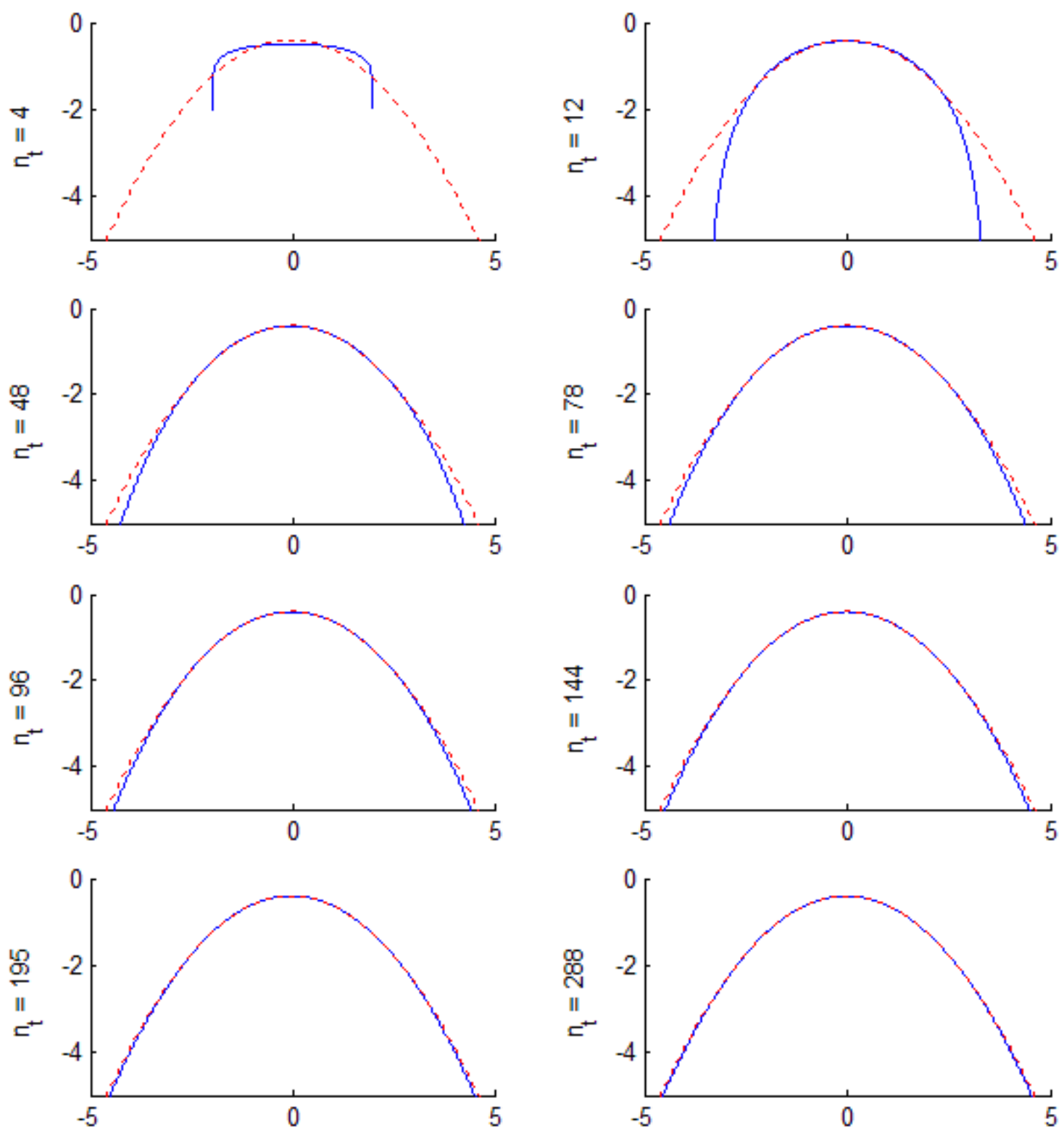

Each of the eight graphs plots the Gaussian pdf (dashed line) against the pdf of the finite sample distribution (solid line) for different values of $n_{t}$. See Appendix I for closed-form expression of the finite sample pdf. 
Figure II. Detected Jumps On Two-Minute Returns In The S\&P 500 Futures Dataset
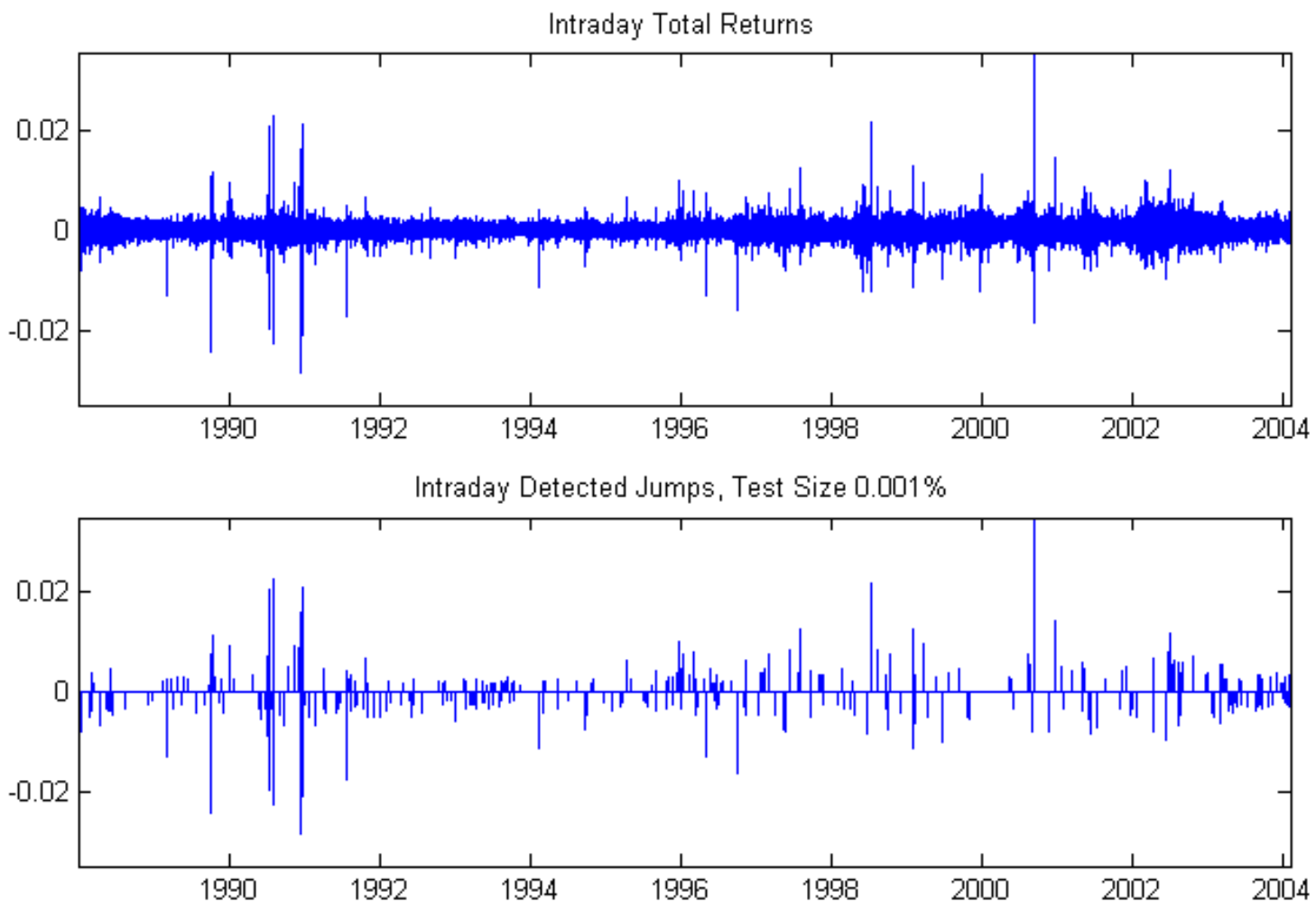

Intraday Diffusive Returns, Test Size $0.001 \%$

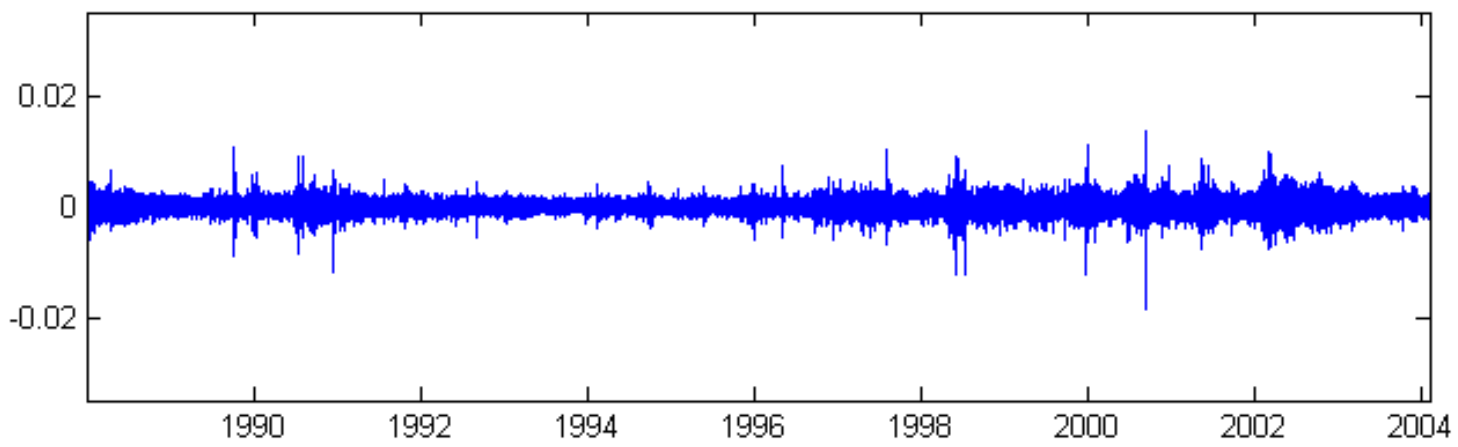

The top graph plots the intraday return series before jump detection.

The middle graph plots the series of detected jumps, following the procedure described in the jump detection section. The bottom graph plots the intraday return series after extracting all detected jumps. 
Figure III.1. Robust Estimation of The First Order Autocorrelation on Two-Minute S\&P500 Returns

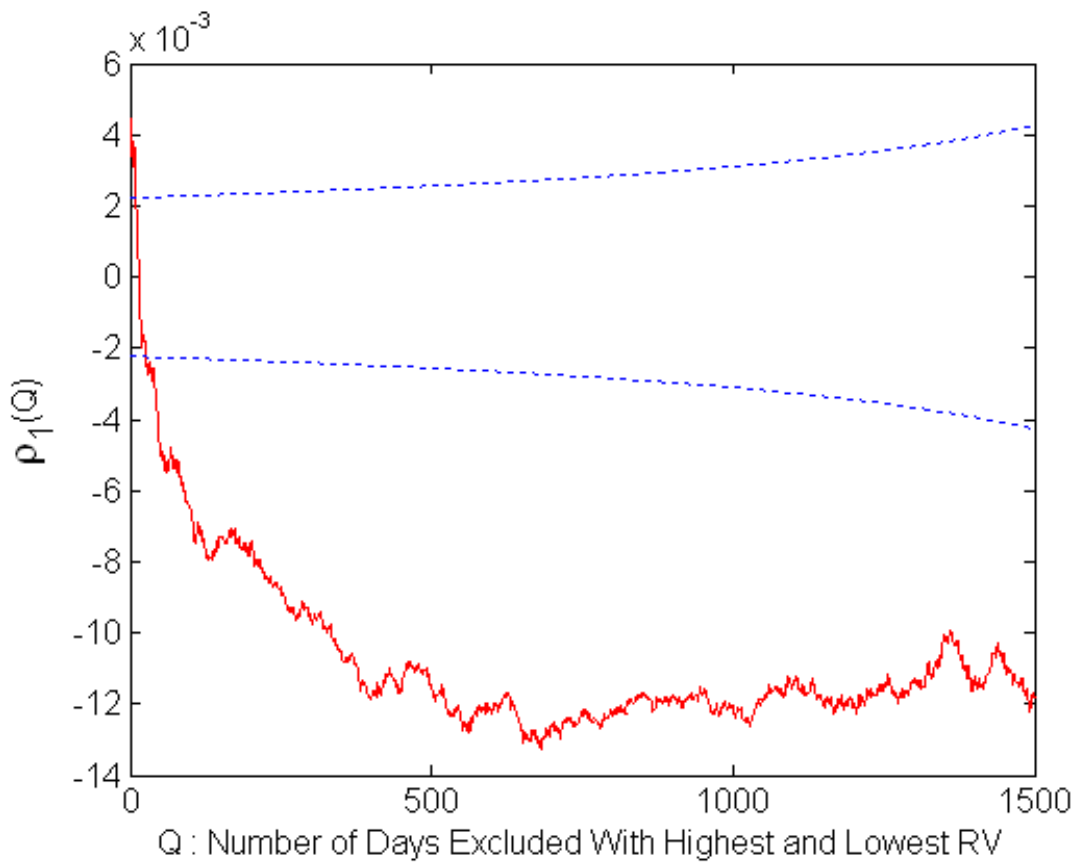

The solid line plots the standard autocorrelation estimate as a function of the number $Q$ of excluded days with highest and lowest realized volatility. Values outside the dashed line bounds indicate significance at $5 \%$ level

Figure III.2. Robust Estimation of The I.I.D. Noise Variance on Two-Minute S\&P500 Returns

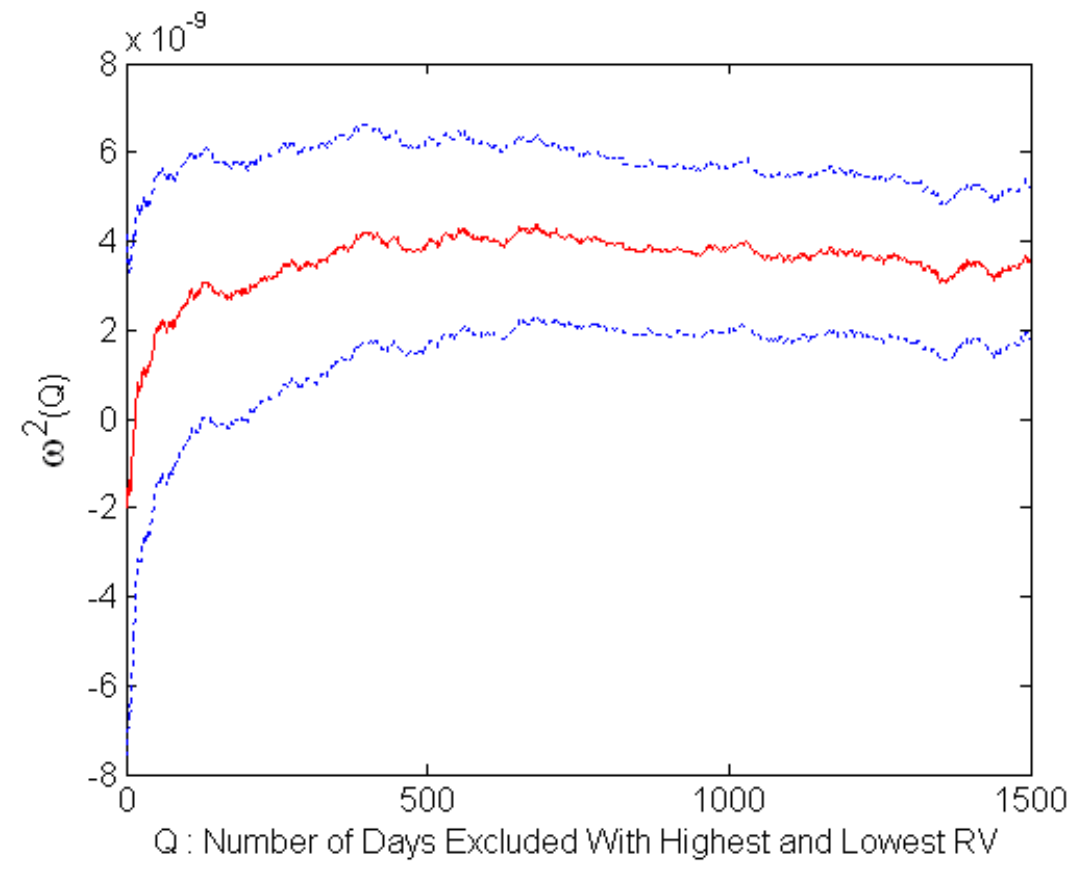

The solid line plots the standard i.i.d. noise variance estimate as a function of the number $\mathbf{Q}$ of excluded days with highest and lowest realized volatility. The dashed lines represent standard $95 \%$ confidence bounds. 